University of Louisville

ThinkIR: The University of Louisville's Institutional Repository

$5-2012$

\title{
Reliability demonstration of a multi-component Weibull system under zero-failure assumption.
}

Markus W. Kemmner 1971-

University of Louisville

Follow this and additional works at: https://ir.library.louisville.edu/etd

\section{Recommended Citation}

Kemmner, Markus W. 1971-, "Reliability demonstration of a multi-component Weibull system under zerofailure assumption." (2012). Electronic Theses and Dissertations. Paper 735.

https://doi.org/10.18297/etd/735

This Doctoral Dissertation is brought to you for free and open access by ThinkIR: The University of Louisville's Institutional Repository. It has been accepted for inclusion in Electronic Theses and Dissertations by an authorized administrator of ThinkIR: The University of Louisville's Institutional Repository. This title appears here courtesy of the author, who has retained all other copyrights. For more information, please contact thinkir@louisville.edu. 


\title{
RELIABILITY DEMONSTRATION OF A MULTI-COMPONENT WEIBULL SYSTEM UNDER ZERO-FAILURE ASSUMPTION
}

\author{
By \\ Markus W. Kemmner \\ Diplom-Ingenieur, Technische Universität Dresden, 1998 \\ Diplom-Wirtschaftsingenieur, FernUniversität Hagen, 2002
}

A Dissertation

Submitted to the Faculty of the

J. B. School of Engineering of the University of Louisville

in Partial Fulfillment of the Requirements for the

Doctor of Philosophy

Department of Industrial Engineering

University of Louisville

Louisville, Kentucky, USA

May 2012 
(C) Copyright 2012 by Markus W. Kemmner

All rights Reserved 


\title{
RELIABILITY DEMONSTRATION OF A MULTI-COMPONENT WEIBULL SYSTEM UNDER ZERO-FAILURE ASSUMPTION
}

\author{
By \\ Markus W. Kemmner \\ Diplom-Ingenieur, Technische Universität Dresden, 1998 \\ Diplom-Wirtschaftsingenieur, FernUniversität Hagen, 2002
}

A Dissertation Approved on

May 9, 2012

By the following Dissertation Committee

Dr. John S. Usher, Committee Chair

Dr. Gail W. DePuy

Dr. William E. Biles

Dr. Thomas Riedel 
To my wife Sibylle.

To Valerie, Timo, Finja, Jule and Frida.

- iii - 


\section{ACKNOWLEDGEMENTS}

First of all I want to express my deepest gratitude to the advisor and committee chair, Dr. John S. Usher, for the opportunity offered to me to pursue this degree. During the entire research program he kindly granted me his support and contributed to the success with open discussions, critical questions and helpful suggestions. He gave special consideration to the situation of advising research work that was mainly done abroad.

I would also like to thank the other committee members, Dr. Gail W. DePuy, Dr. William E. Biles and Dr. Thomas Riedel for their assistance and guidance during the development of this research work.

I want to thank LINDO $^{\circledR}$ Systems Inc. for temporarily providing free access to an unrestricted software license of LINGO ${ }^{\mathrm{TM}}$. The mentioned software package was utilized successfully within the research work conducted for this dissertation. I also want to thank Dr. DePuy and the computer technicians from the Department of Industrial Engineering for their support while receiving the license.

This research was part of the German PhD program of the Department of Industrial Engineering of the University of Louisville which was initiated in 2009. I want to thank all faculty members who have adapted in a very flexible way to the needs of a distance learning program during their courses for this program. 


\title{
ABSTRACT \\ RELIABILITY DEMONSTRATION OF A MULTI-COMPONENT WEIBULL SYSTEM UNDER ZERO-FAILURE ASSUMPTION
}

\author{
Markus W. Kemmner
}

May 9, 2012

This dissertation is focused on finding lower confidence limits for the reliability of systems consisting of Weibull components when the reliability demonstration testing (RDT) is conducted with zero failures. The usual methods for the parameter estimation of the underlying reliability functions like maximum likelihood estimator (MLE) or mean squares estimator (MSE) cannot be applied if the test data contains no failures. For single items there exists a methodology to calculate the lower confidence limit (LCL) of reliability for a certain confidence level. But there is no comparable method for systems.

This dissertation provides a literature review on specific topics within the wide area of reliability engineering. Based on this and additional research work, a first theorem for the LCL of system reliability of systems with Weibull components is formulated. It can be applied if testing is conducted with zero observed failures. This theorem is unique in that it allows for different Weibull shape parameters for components in the system. The model can also be applied if each component has been exposed to different test durations. This can result from accelerated life testing (ALT) with test procedures that have different acceleration factors for the various failure modes or components respectively. A second theorem for $B_{X}$ - lifetime, derived from the first theorem, has been formulated as well. 
The first theorem on LCL of system reliability is firstly proven for systems with two components only. In the following the proof is extended towards the general case of $n$ components. There is no limitation on the number of components $n$. The proof of the second theorem on $B_{X}$ - lifetime is based on the first proof and utilizes the relation between $B_{X}$ and reliability.

The proven theorem is integrated into a model to analyze the sensitivity of the estimation of the Weibull shape parameter $\beta$. This model is also applicable if the Weibull parameter is subject to either total uncertainty or of uncertainty within a defined range.

The proven theorems can be utilized as the core of various models to optimize RDT plans in a way that the targets for the validation can be achieved most efficiently. The optimization can be conducted with respect to reliability, $B_{X}-$ lifetime or validation cost. The respective optimization models are mixed-integer and highly non-linear and therefore very difficult to solve. Within this research work the software package LINGO $^{\mathrm{TM}}$ was utilized to solve the models. There is a proposal included of how to implement the optimization models for RDT testing into the reliability process in order to iteratively optimize the RDT program based on failures occurred or changing boundary conditions and premises.

The dissertation closes with the presentation of a methodology for the consideration of information about the customer usage for certain segments such as market share, annual mileage or component specific stress level for each segment. This methodology can be combined with the optimization models for RDT plans.

Key words:

System Reliability - Series System - Reliability Demonstration Testing Weibull Distribution - Accelerated Lifetime Testing - Lower Confidence Limit Confidence Level - Lower Confidence Bound - Zero Failure - Success Run 


\section{TABLE OF CONTENTS}

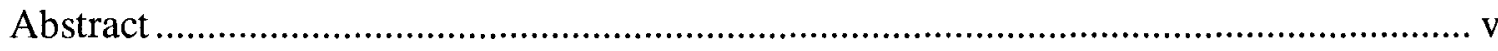

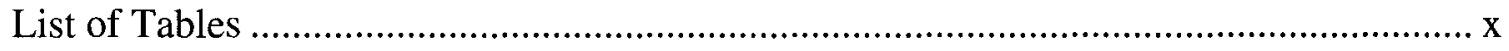

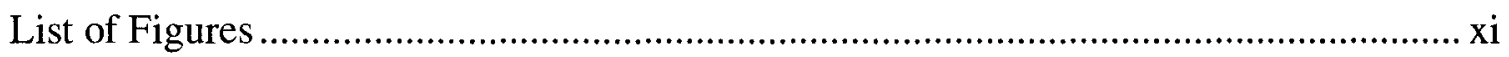

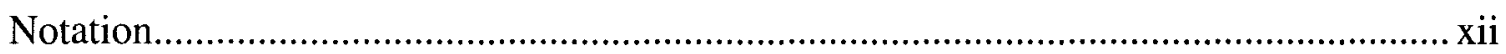

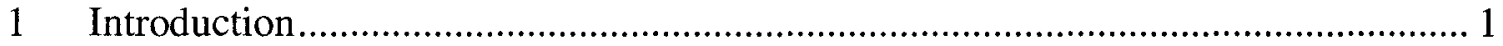

$1.1 \quad$ Reliability Demonstration Testing .............................................................. 1

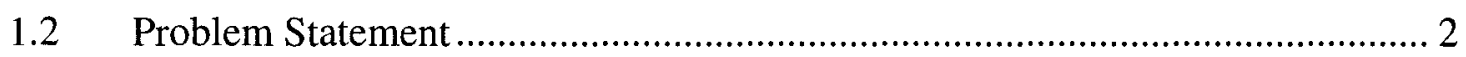

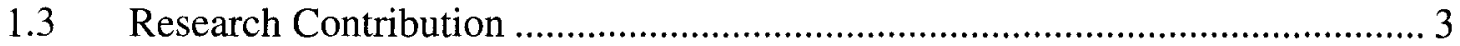

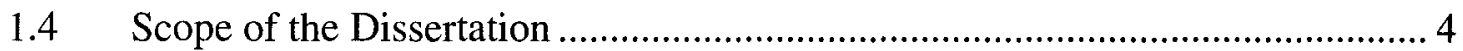

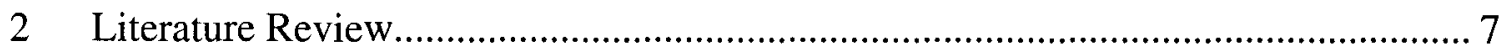

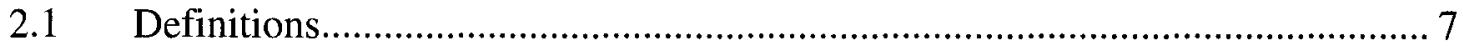

2.1.1 Reliability and Lifetime Analysis ......................................................... 7

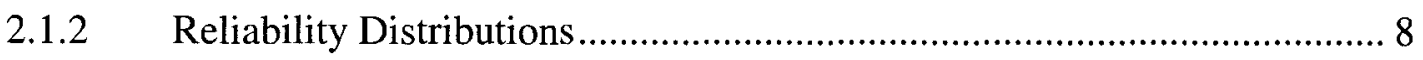

2.2 Reliability of Systems ......................................................................... 12

2.3 Reliability Growth and Reliability Demonstration Testing .............................. 14

2.4 Type I and Type II Error in Reliability Demonstration Testing ....................... 18

$2.5 \quad$ Reliability Function Estimation from Test Data ............................................... 23

2.5.1 Reliability Estimation Based on Test Data Including Failures................. 24

2.5.2 Determination of LCL of Reliability from Data with No Failure............. 27

2.6 Influence of RDT on Product Life Cycle Cost ................................................ 32

$2.7 \quad$ Accelerated Lifetime Tests (ALT) ............................................................. 34

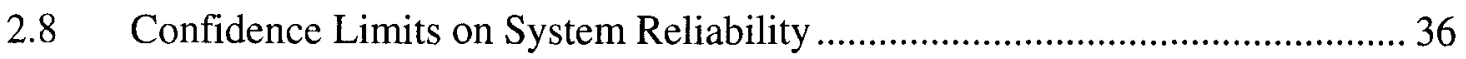

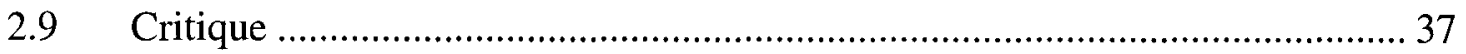

3 Framework for System Reliability Estimation with Zero-Failure Data...................... 38

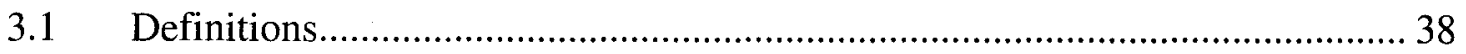

3.2 Assumptions and Premises ......................................................................... 43

3.3 First Theorem - LCL of Reliability of a System with Zero-Failure Data........ 45 
3.4 Second Theorem - LCL of $B_{X}$ - lifetime of a System with Zero-Failure Data 47

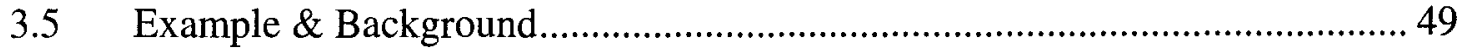

3.6 Advantages of the Proposed Method to Calculate LCL of System Reliability 55

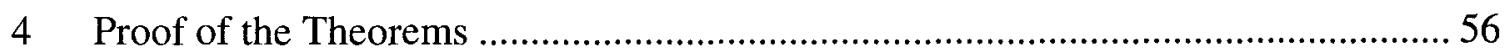

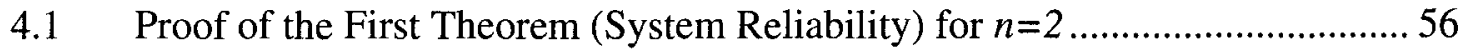

4.2 General Proof of the First Theorem (System Reliability) ( $n$ Components) ..... 62

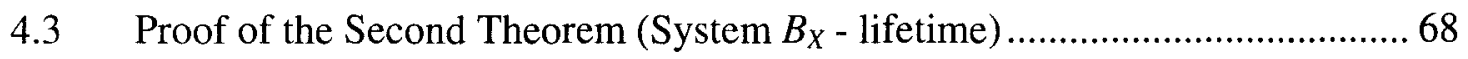

5 Sensitivity of the Weibull Shape Parameter Estimation ........................................ 71

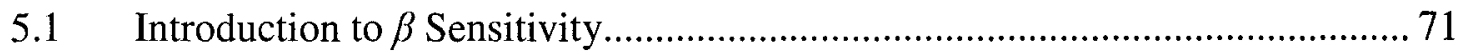

5.2 Optimization Model to Consider $\beta$ Sensitivity ........................................... 72

5.3 Application of the Model to Consider $\beta$ Sensitivity (Example) ...................... 76

5.4 Remark on Efficient Optimization Model Solution....................................... 82

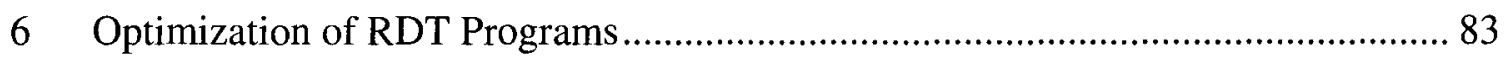

6.1 Relevant Cost Information for Decision Making......................................... 83

6.2 Model for Confidence Limit of Total Cost Influenced by Validation .............. 84

6.3 Model for Optimizing RDT Plans with Respect to the LCL of Reliability ...... 87

6.4 Application of the RDT Optimization Model (example)................................ 90

6.5 Model for Optimizing RDT Plans with Respect to Cost ............................. 96

6.6 Model for Optimizing RDT Plans with Respect to LCL of $B_{X}$ - lifetime ....... 99

6.7 Disadvantages of Identical Test Duration for all Units ................................ 103

6.8 Integration of RDT Optimization into the Reliability Growth Process .......... 111

7 Considering Different Customer Segments and the Respective Load Factors ....... 113

7.1 Calculation of Cumulative Reliability Across Different Segments with

Total Reliability ................................................................................... 113

7.2 Calculating Reliability Considering Component Specific Stress Factors...... 116

7.3 Model for Cumulative Reliability Across Different Customer Segments ..... 117

7.4 Example for Consideration of Customer Usage Specific Stress Factors ........ 118

7.5 Integration of Customer Segment Consideration into RDT Optimization .... 124

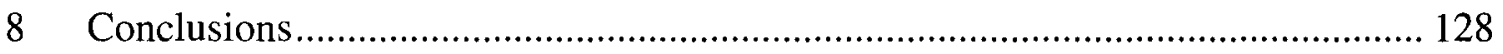

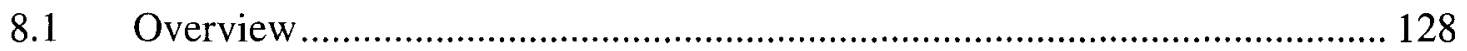

8.2 Contributions to the Body of Knowledge ............................................. 130 


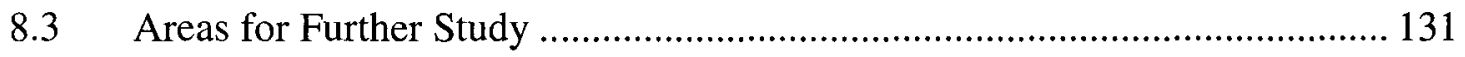

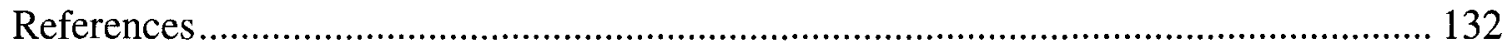

Appendix 1 - The Weibull Distribution ...................................................................... 137

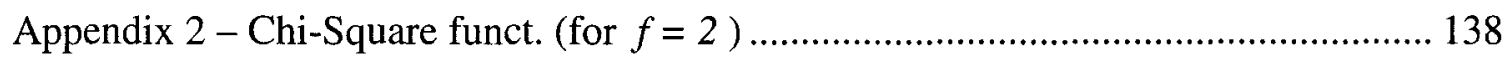

Appendix 3 - Reliability of a System with $n$ Identical Components.............................. 139

Appendix 4-LCL of Reliability of Single Weibull Items With Zero Failures ............ 142

Appendix 5 - On LCL of System Reliability When Failures Have Occurred in RDT .. 145

Appendix 6 - Applied Computer System to Solve Optimizations ................................... 152

Appendix 7 - List of Abbreviations........................................................................... 153

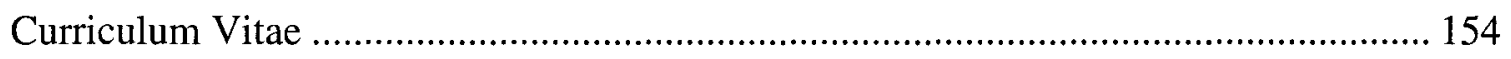




\section{LIST OF TABLES}

Table 2.1 Type I error and Type II error in hypothesis testing .................................. 19

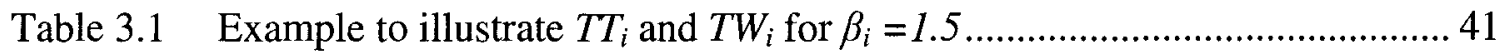

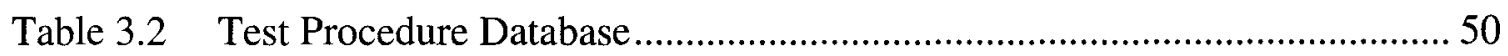

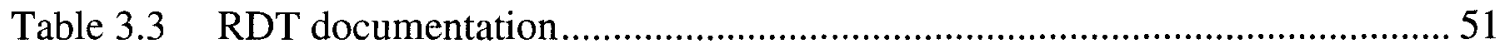

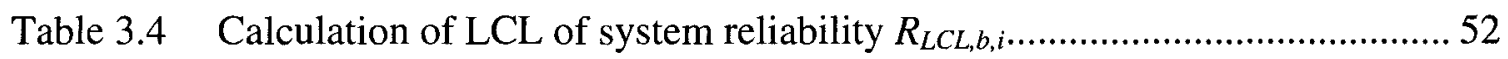

Table 3.5 Calculation of $\mathrm{b}=10 \% \mathrm{LCL}$ of system $B_{X}$ - lifetime .................................... 55

Table 5.1 Comparison of fixed values for $\beta_{i}$ vs. upper and lower bound .................... 76

Table 5.2 LCL of system reliability at $t_{0}=400$ with variable and fixed $\beta_{i} \ldots \ldots \ldots \ldots \ldots . . . .78$

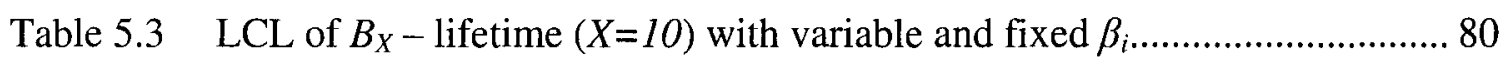

Table $5.4 \quad b=0.1$ LCL of system reliability $\left(t_{0}=400\right)$ with unrestricted $\beta_{i} \ldots \ldots \ldots \ldots \ldots \ldots . . . . . .22$

Table 6.1 RDT optimization: documentation of already finished tests ....................... 90

Table 6.2 RDT optimization: $T W_{F i}$ and $R_{S ; L C L ; b}$ based on the finished tests .............. 91

Table 6.3 RDT optimization: test procedure database - acceleration factors.............. 91

Table 6.4 RDT optimization: test procedure database - constraints and cost .............92

Table 6.5 RDT optimization: optimized validation program.................................... 93

Table 6.6 RDT optimization: achieved LCL of system reliability with add. testing.... 94

Table 6.7 RDT optimization: achieved reliability depending on budget .................... 94

Table 6.8 Demonstrated LCL of reliability prior to additional RDT........................ 104

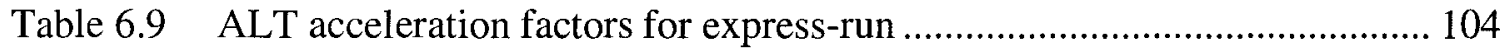

Table 6.10 Constraints and Cost for Express-Run ................................................. 105

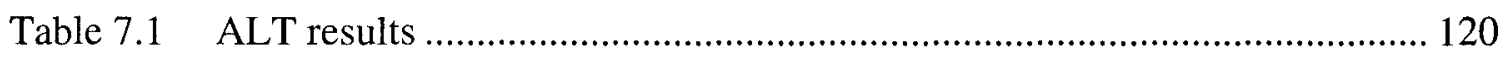

Table 7.2 Customer segments, their annual mileage and market share .................... 121

Table 7.3 Stress Factors for each component in all customer segments................... 122

Table 7.4 Cumulative LCL of component and system reliability........................... 123 


\section{LIST OF FIGURES}

Figure 1.1 Framework for system reliability estimation w/ zero failures - overview .. 6

Figure 2.1 Serial and parallel systems with $n$ components ..................................... 12

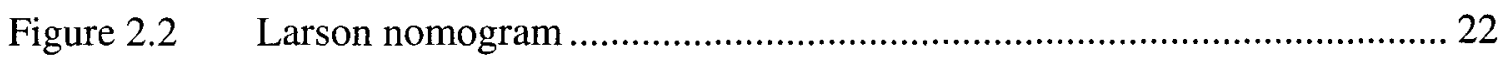

Figure 2.3 Theoretical product development cost versus reliability curve ................ 33

Figure 2.4 ALT - failure distribution (paperclip bending test with diff. angles) ....... 35

Figure 3.1 LCL of system and component reliability ........................................ 54

Figure 4.1 Probability of zero failure in RDT at different system reliability levels .. 57

Figure 4.2 Illustration of the theorem for LCL of system reliability ........................58

Figure 4.3 Reliability of a serial system with Weibull components ......................... 63

Figure 4.4 LCL for system reliability as the minimum of LCLs of component

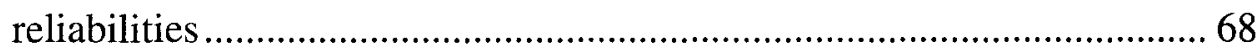

Figure 4.5 LCL of system $B_{l 0}$ as the minimum of the LCLs of component $B_{10} \ldots \ldots .69$

Figure 5.1 Comparison of $b$ LCL of system reliability with fixed and variable $\beta_{i} \ldots .77$

Figure 5.2 Minimum of $b$ LCL of system reliability with unrestricted $\beta_{i} \ldots \ldots \ldots \ldots \ldots . . . . .81$

Figure 6.1 LCL of reliability and UCL for warranty and total cost ........................ 86

Figure 6.2 LCL of system reliability for different validation budgets .................... 96

Figure 6.3 Relationship between LCL of system reliability and validation cost ..... 105

Figure 6.4 Advantage of modified optimization policy ....................................... 106

Figure 6.5 Integration of RDT Optimization into the Reliability Growth Process .. 111

Figure 7.1 Example for the consideration of reliability of customer segments ....... 114

Figure A1.1 Diagrams of the Weibull distribution (Reliability, CDF, pdf).............. 137

Figure A5.1 MCS result: the potential LCL of system reliability is not adequate...... 150 


\section{NOTATION}

$A F_{i j} / A F_{P i k} \quad$ acceleration factor for component $i$ in test $j /$ in planned test $k$

a confidence level, probability of Type I error, producer's risk

$B_{X}, B_{10} \quad B_{X}-$ lifetime $/ B_{10}-$ lifetime i.e. operating time at which a maximum share of $X \% / 10 \%$ respectively will have failed

$B_{X ; C i ; L C L ; b} \quad b$ LCL of $B_{X}$ - lifetime of component $i$

$B_{X ; S ; L C L ; b} \quad b$ LCL of system $B_{X}$ - lifetime

$b \quad$ confidence level, probability of Type II error, consumer's risk

$C_{f k}, C_{v k} \quad$ fixed cost (per test), variable cost (per unit of duration) of test type $k$

$C_{t k} \quad$ total cost of test type $k$

$C_{t}, C_{t \max } \quad$ validation budget, maximum validation budget

C confidence level

CF correction factor for the case of failures during RDT

$d_{A l} \quad$ annual mileage of usage according to customer segment $l$

$F(t) \quad$ cumulative (failure) distribution function (CDF)

$f(t) \quad$ probability density function (pdf)

$h(t) \quad$ hazard rate

$L($ ) likelihood function

$L R \quad$ lifetime ratio

$l, l_{\max } \quad$ indicator for customer segment / number of different customer segments

$l_{P k} \quad$ number of planned tests of type $k$

$l_{P, \min k}, l_{P, \max k} \quad$ lower and upper limit for the number of planned tests of type $k$

$m, m_{i} \quad$ number of tests, number of systems on test from component $i$ 


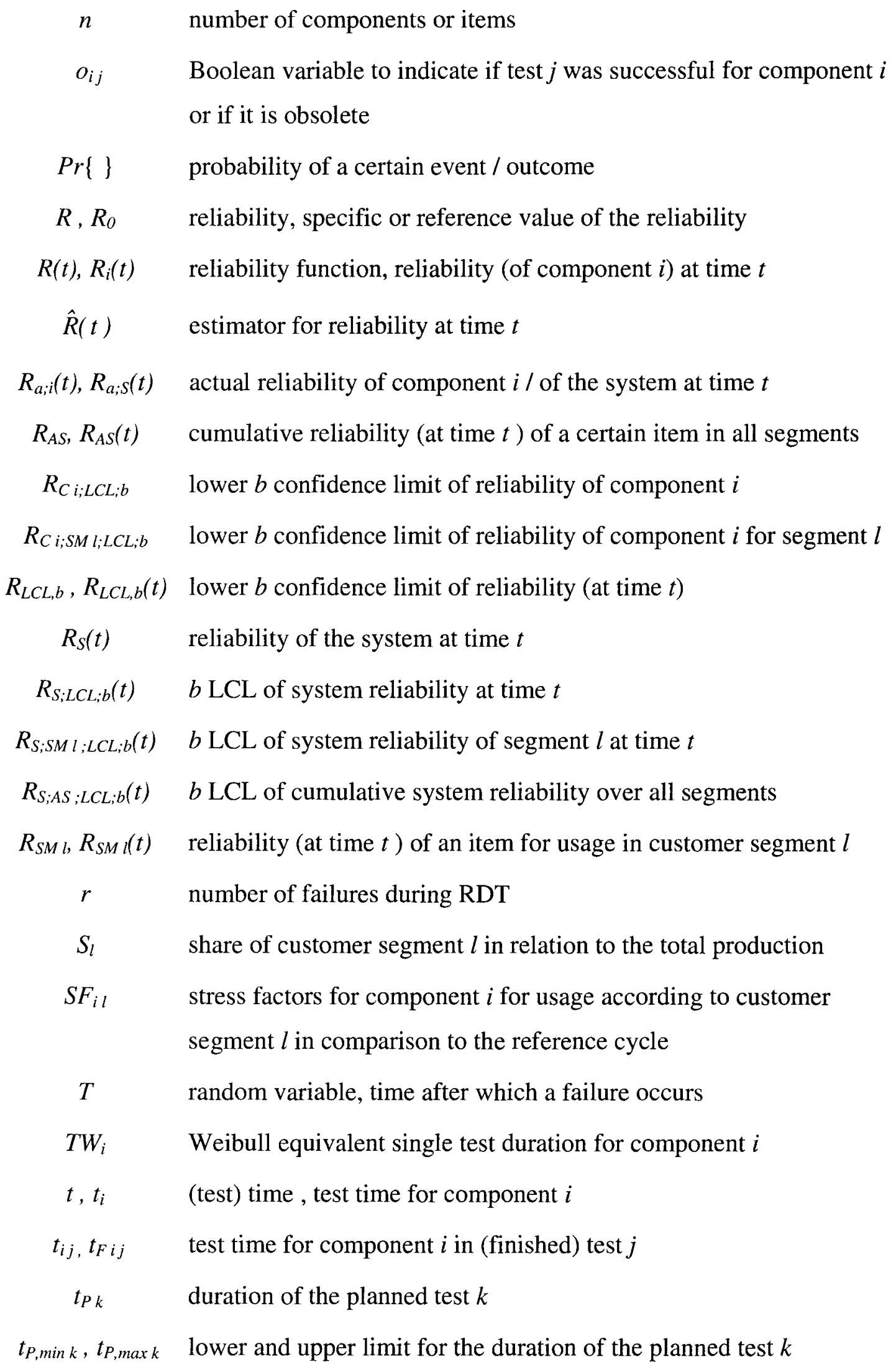




$\begin{array}{ll}v_{P k} & \text { calculatory speed of the planned test } k \\ Z_{1-a} & (1-a)-\text { percentile of the Z-statistic (standard normal distribution) } \\ \beta, \beta_{i} & \text { shape parameter of the Weibull distribution (for component } i \text { ) } \\ \beta_{o p t ; i} & \text { shape parameter that leads to the lowest value of the LCL of } \\ & \text { component reliability (for component } i \text { ) } \\ \theta, \theta_{i} & \quad \begin{array}{l}\text { scale parameter of the Weibull distribution (for component } i \text { ) } \\ \theta_{a ; i}\end{array} \\ \chi_{b ; f}^{2} & \quad \begin{array}{l}\text { actual scale parameter of the Weibull distribution (for component } i \text { ) } \\ \text { th }\end{array}\end{array}$




\section{INTRODUCTION}

\subsection{Reliability Demonstration Testing}

Quality and reliability are very important attributes of a product as they are often the key purchasing criteria for most customers. Their importance is increasing since the respective expectations of the customers are growing as well.

In addition to creating disappointed customers, a lack of product quality and reliability leads to direct warranty cost in case where the failure occurs during the warranty period. If the failure is safety related, a product recall might be necessary to avoid litigation due to product liability.

A low level of quality and reliability can also cause other effects and indirect costs, for example unsatisfied customers communicating to other potential customers, or negative effects on the brand image by reports in the media on quality problems or on product recalls.

Therefore, it is of interest for every company to ensure a high level of quality and reliability to safeguard its future marketing position and profitability. Each quality or reliability issue of a new product should be discovered and fixed - the sooner the better, and the cheaper.

But one can also test too much. Reliability growth and reliability demonstration testing to ensure the high standard of quality and reliability are expensive and increase the time to market. A significant share of the total R\&D-budget for durable products like automobiles goes into reliability growth and reliability demonstration testing. 
There is a clear motivation to test to the right extent. Too much testing leads to high R\&D and thus life cycle cost. Too little testing puts a high financial risk on a company. Regardless of the absolute level of reliability testing, it is the task of the quality engineer to ensure that the time and money spent for reliability growth and demonstration creates the best possible effect on reliability and thus generates the highest return on investment.

\subsection{Problem Statement}

If a large sample is tested, inferences on the reliability and lifetime distributions of the entire population are possible. But testing a large sample is expensive especially if the items are tested until they fail.

If smaller sample sizes are tested, there is only a limited statistical significance of the derived statements on the population's reliability and lifetime distribution. For the automotive industry it is typical that only a very limited sample size is put on test for reliability demonstration since the prototypes of a new vehicle type are very expensive. Due to the limited sample size, no failures at all are accepted during RDT - each failure that occurs leads to a corrective countermeasure. So the final design released for production has technically had no failures during RDT. Often some components at the final design level are only exposed to a small amount of testing time in the case where they have experienced a design change late in the validation phase of the development process.

For the special case in which zero failures occur during testing, it is not possible to estimate the reliability with generally accepted methods like maximum likelihood estimator (MLE) or mean square estimator (MSE). But for single components a lower confidence limit (LCL) of reliability can be calculated based on the testing durations the system was exposed to. Typically such a LCL of reliability is relatively low. 
If system reliability of a serial system is then calculated by multiplying these already low LCL of component reliability, the result will be an unrealistic low value for the LCL of the system reliability.

There is no method to calculate a more realistic LCL of system reliability, especially if the individual components are tested with different durations. This can happen for example if RDT is conducted as Accelerated Lifetime Testing (ALT) and the acceleration factors for each component are different.

\subsection{Research Contribution}

This dissertation will present a methodology for calculating the LCL of the reliability of a system in a serial configuration when zero failures have occurred during RDT. The premise of this model is that all components of the system are Weibull and that the Weibull parameter $\beta$ of each component is known or can be estimated. It is assumed that the components can be exposed to different effective testing durations. Comparable methods exist so far only for individual components, not for systems. A theorem will be proposed and proved to demonstrate the method.

A second theorem, derived from the first, to calculate the system LCL of $B_{x}$-lifetime of the system will be presented as well.

Based on these theorems and the respective methodology, a model to calculate the

minimum system reliability for the case that the component Weibull parameters are subject to uncertainty or within a certain range is also included. 
The methodology can be the core of derived optimization models for optimizing the RDT program to achieve a certain level of reliability demonstration at the lowest cost. Such an optimization model is included in the dissertation as well. Furthermore a proposal for integrating the RDT optimization into the reliability growth process is presented.

Finally an enhanced methodology to consider different component-specific stress factors in the various customer segments is introduced.

\subsection{Scope of the Dissertation}

This dissertation is organized in the following way. Chapter 2 contains a literature review on different aspects of reliability starting with important definitions. The main focus is on reliability demonstration testing especially for zero-failure data. Reliability of systems, Accelerated Lifetime Testing (ALT) and Monte Carlo Simulation (MCS) are included as well. The literature review deals with classical probability models and does not include Bayesian methods.

In Chapter 3, a framework for estimating reliability of a series system for the case that no failures occurred during RDT is presented. The methodology is based on two theorems: on $b \mathrm{LCL}$ of system reliability as well as on $b \mathrm{LCL}$ of system $B_{X}$ - lifetime. It is applicable to systems consisting of Weibull components with different or identical Weibull parameters. The necessary assumptions the method is based on, are presented as well. An example illustrates the application of the method.

Chapter 4 gives the mathematical proof of the two theorems. The proposed methods can be applied for serial systems with Weibull components if zero failures occur during RDT. The mathematically proven theorems are the core of the versatile framework around system reliability estimation, which is presented in the following chapters. 
Chapter 5 presents a model to consider the sensitivity of incorrect estimations of the component Weibull shape parameters that extend the introduced theorems. Applying this model is more conservative than the direct application of the theorems of Chapter 3 since it leads to lower LCL of system reliability.

The proven theorems can be integrated into models to optimize RDT programs as explained in detail in Chapter 6. The optimization can take place with respect to LCL of system reliability as well as with respect to LCL of $B_{X}$ - lifetime or with respect to validation cost.

Chapter 6.8 shows how the optimization models, which were introduced before, can be integrated into the iterative reliability growth process in a way that not only the tested system is updated if failures occur but also the RDT program optimization is updated.

Finally Chapter 9 proposes a methodology for considering information about different customer usage. The different customers are grouped into segments. For each of these segments there is information about annual mileage and the respective load on the different components of the system in comparison to the reference customer usage. This methodology can be combined with RDT optimization model as presented in Chapter 6 .

An overview of the different components of the complete RDT framework presented in this dissertation is given in Figure 1.1. 


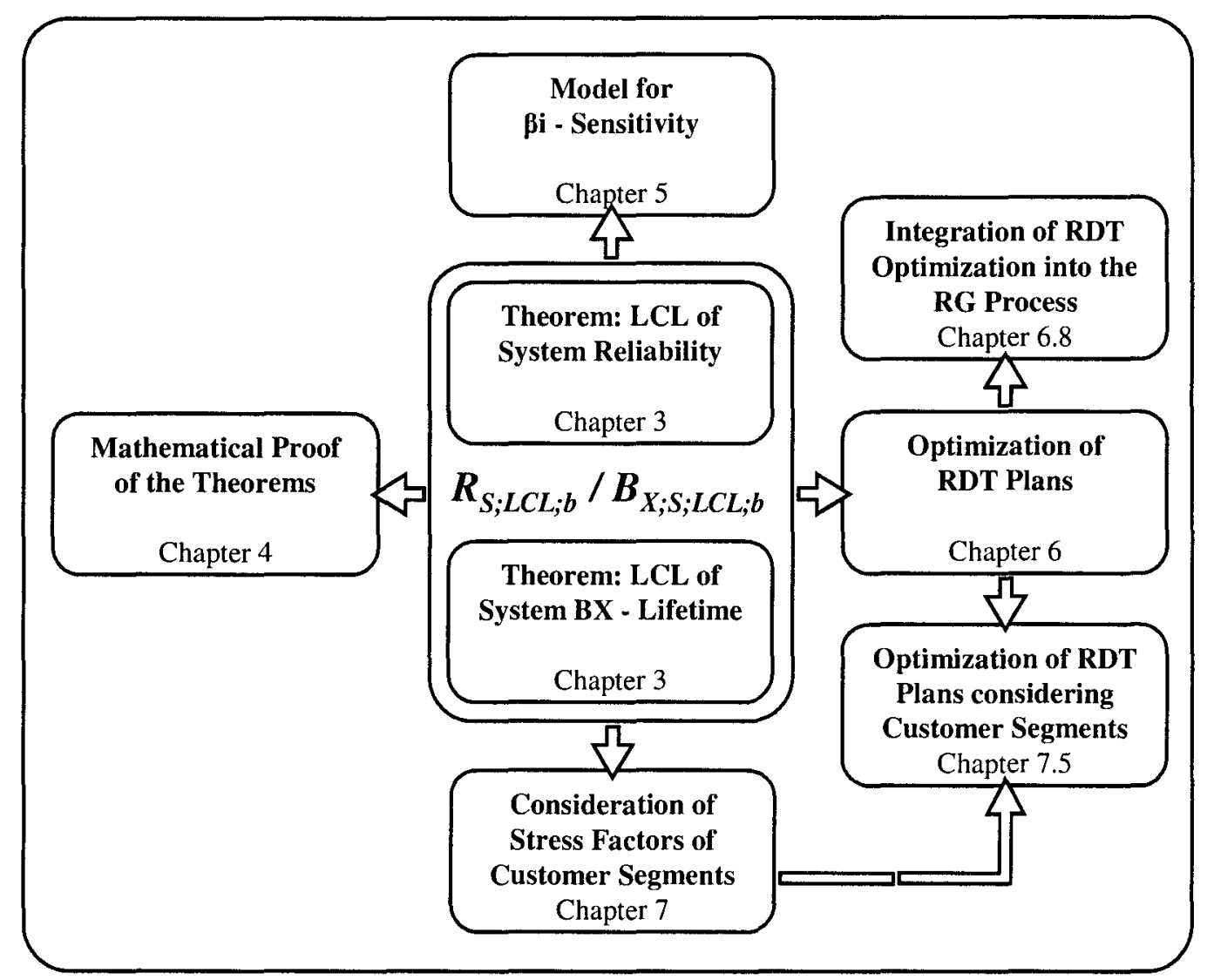

Figure 1.1 Framework for system reliability estimation w/ zero failures - overview 


\section{LITERATURE REVIEW}

Basic definitions and relations that will be important in the further course of the dissertation are presented in the literature review as well as the current state of research and literature in selected fields of reliability engineering.

The literature review is structured in the following way:

- definitions of reliability, reliability distributions

- reliability of systems

- reliability demonstration testing and reliability growth

- estimation of reliability or of parameters of reliability functions

- accelerated lifetime testing

- influence of reliability demonstration testing on life cycle cost

- lower confidence limits of system reliability

\subsection{Definitions}

\subsubsection{Reliability and Lifetime Analysis}

Since the latter half of the $20^{\text {th }}$ century there has been a lot of research activity in reliability and maintainability engineering. The US military was one of the drivers of these activities and introduced important definitions in the Military Handbook (2009). This research presented here will utilize these definitions. 
Reliability is defined as "the probability that an item will perform its intended function for a specified time and under stated conditions"1. For a high population or number of identical systems considered, reliability at time $t$ is the ratio between the number of systems still in operation after that time to the total number of systems $n$ in the beginning of the observation.

The symbol $T$ denotes the lifetime of a specific item or the time of the occurrence of a certain failure mode. In this context an item could either be a system or a component. $T$ could also denote another type of duration for example a vehicle mileage. The lifetime $T$ is a positive and continuous random variable, where $T>0$.

\subsubsection{Reliability Distributions}

The reliability function or reliability distribution $R(t)$ describes the probability of failurefree operation until operating time $t$.

$R(t)=\operatorname{Pr}\{T>t\}$

Reliability distributions are only defined for positive values of $t$ (i.e. $t>0$ ). It is assumed that at the time $t=0$ all systems or components of a certain population are working properly without any failure:

$R(0)=1$

The reliability function is monotonic decreasing (since components can only fail and there is no healing or repair of failed components considered). The useful life or maximum operating time of the components is limited. Thus at infinite time all units will have failed:

$\lim _{t \rightarrow \infty} R(t)=0$

${ }^{1}$ Military Handbook (2009) p. 3 
The cumulative failure distribution or the cumulative distribution function (CDF) $F(t)$ describes the probability of failure after time $t$. This is the complement of the reliability since a failure either has yet occurred or not yet occurred.

$F(t)=\operatorname{Pr}\{T \leq t\}=1-R(t)$ for $t>0$

The first derivative of the CDF with respect to time $t$ is called probability density function (pdf):

$f(t)=\frac{d F(t)}{d t}=-\frac{d R(t)}{d t} \quad$ for $t>0$

The pdf by definition is non-negative and the area under the pdf curve is equal to $l$ :

$f(t) \geq 0 \quad$ for $t>0$

$\int_{0}^{\infty} f(t) d t=1$

For any given time $t$ the reliability $R(t)$ is the area under the pdf curve above $t$ (up to $\infty$ ). $\mathrm{CDF} F(t)$ is the area under the pdf between zero and $t$ respectively.

$$
\begin{aligned}
& R(t)=\int_{t}^{\infty} f(t) d t \\
& F(t)=\int_{0}^{t} f(t) d t
\end{aligned}
$$

There are various basic probability distributions used in reliability. The most common ones are:

- Weibull distribution

- log-normal distribution

- normal distribution

- exponential distribution 
The Weibull distribution is used very often to describe reliability because many different failure characteristics of real-world systems and components can be fitted to a Weibull ${ }^{2}$ function. The distribution function has the following two parameters:

- shape parameter $\beta$

- scale parameter $\theta$

The distribution is defined by the reliability function ${ }^{3}$ :

Reliability function: $R(t)=e^{-\left(\frac{t}{\theta}\right)^{\beta}}$ for $t, \theta, \beta>0$

Thus the CDF and pdf of the Weibull reliability function are:

$\mathrm{CDF}: F(t)=1-R(t)=1-e^{-\left(\frac{t}{\theta}\right)^{\beta}}$ for $t, \theta, \beta>0$

pdf: $f(t)=\frac{d F(t)}{d t}=-\frac{d R(t)}{d t}=\frac{\beta t^{\beta-1}}{\theta^{\beta}} e^{-\left(\frac{t}{\theta}\right)^{\beta}}$ for $t, \theta, \beta>0$

The hazard rate $h(t)$ is the share of systems failing in a certain period of time from the number of system being still in service at the beginning of the considered period. For the Weibull distribution, the hazard rate is:

$h(t)=\frac{f(t)}{R(t)}=\frac{\beta t^{\beta-1}}{\theta^{\beta}}$ for $t, \theta, \beta>0$

For $\beta=1$ the hazard rate $h(t)$ is constant over operating time $t$. This means that the system does not experience wear, aging or other forms of degradation over lifetime. All of these would lead to an increasing failure rate. So for $\beta=l$ the failures occur only driven by pure randomness. For this special case with $\beta=1$ the Weibull distribution is identical to the exponential distribution.

\footnotetext{
${ }^{2}$ named after E. H. Waloddi Weibull (1887 - 1979), Swedish mathematician and engineer

${ }^{3}$ Pham (2006) p. 6
} 
The shape parameter $\beta$ significantly determines the reliability behavior over the item's lifetime.

- For $\beta<1$ both hazard rate and pdf are constantly decreasing.

This means that most failures occur for a new system when it is set into operation or shortly after.

- For $\beta>l$ the hazard rate is constantly increasing. This is a typical behavior if a system has wear or other forms of degradation.

Appendix 1 presents diagrams of reliability $R(t), \operatorname{CDF} F(t), \operatorname{pdf} f(t)$ and hazard rate $h(t)$ for Weibull with different parameters $\beta$ and $\theta$. Reasons for the wide usage of the Weibull distribution are the possibility to find mathematical solutions for the integral of $R(t)$ and $F(t)$ as well as the wide flexibility to fit the parameters to existing characteristics of real processes and failure data.

This research work is focused on systems consisting of components whose failure characteristics can be modeled with a Weibull distribution. Methods developed using Weibull are also applicable to the exponential distribution (with $\beta=1$ ).

\section{$\underline{B} \underline{x}$-Lifetime $\left(\underline{B}_{10} \underline{\text {-Lifetime })}\right.$}

The lifetime $B_{X}$ is defined as the time at which not more than $X \%$ of all parts of a certain population will have failed. $B_{X}$ is the inverse function of the CDF and is thus closely related to the reliability function $R(t)$.

$$
R\left(B_{X}\right)=(100-X) \% \text { i.e. } R\left(B_{10}\right)=0.9
$$

For Weibull with (2.10):

$$
B_{X}=\theta *[-\ln (100 \%-X \%)]^{1 / \beta} \text { i.e. } B_{10}=\theta *[-\ln 0.9]^{1 / \beta}
$$




\subsection{Reliability of Systems}

Systems generally consist of various components. Components are often arranged in two basic configurations, as illustrated in Figure 2.1:

- Serial configuration

- Parallel configuration

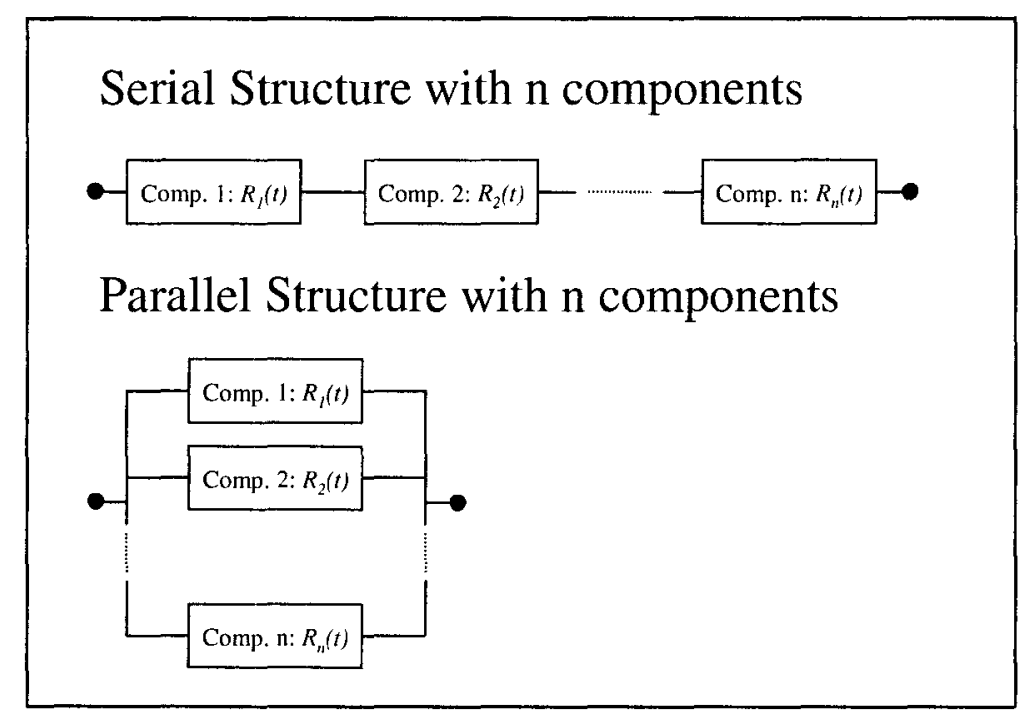

Figure 2.1 Serial and parallel systems with $n$ components

$\underline{\text { Serial system structure: }}$

All components have to work simultaneously to ensure the system functionality. Failure of any component leads to failure of the system. This is related to a situation where each component fulfills a specific function. Most systems within automotive systems are considered to have a serial configuration. The reliability of a system $R_{S}$ with serial structure is always lower than the lowest reliability of an individual component since this reliability is equal to the probability of all components working successfully at the same time: 


$$
\begin{aligned}
& R_{S}(t)=\prod_{i=1}^{n} \operatorname{Pr}\left(T_{i}>t\right)=\prod_{i=1}^{n} R_{i}(t) \\
& R_{S}(t) \leq \operatorname{Min}\left\{R_{i}(t)\right\}
\end{aligned}
$$

This relation holds when the failure of individual components are assumed to be independent.

The reliability of a serial system consisting only of Weibull components with parameters $\beta_{i}$ and $\theta_{i}$ is $^{4}$ :

$$
\begin{aligned}
& R_{S}(t)=\prod_{i=1}^{n} R_{i}(t)=\prod_{i=1}^{n} e^{-\left(\frac{t}{\theta_{i}}\right)^{\beta_{i}}}=e^{-\left(\frac{t}{\theta_{l}}\right)^{\beta_{1}}} e^{-\left(\frac{t}{\theta_{2}}\right)^{\beta_{2}}} . . e^{-\left(\frac{t}{\theta_{n}}\right)^{\beta_{n}}}=e^{-\sum_{i=\lambda}^{n}\left(\frac{t}{\theta_{i}}\right)^{\beta_{i}}} \\
& -\ln \left[R_{S}(t)\right]=\sum_{i=1}^{n}\left(\frac{t}{\theta_{i}}\right)^{\beta_{i}}=\left(\frac{t}{\theta_{1}}\right)^{\beta_{1}}+\left(\frac{t}{\theta_{2}}\right)^{\beta_{2}}+\ldots . .+\left(\frac{t}{\theta_{n}}\right)^{\beta_{n}}
\end{aligned}
$$

This research work will only consider systems in serial configuration.

\section{Parallel system structure:}

The system reliability can be increased when there is redundancy added and various components with the same function are arranged in a parallel configuration. In real technical systems redundancy can be found in situations where failures can cause very critical damage like in nuclear power plants or aircraft.

The reliability of the parallel system is equal to 1 minus the probability of all components failing at the same time. The reliability of a parallel system is always higher than the highest reliability of an individual component.

\footnotetext{
${ }^{4}$ see Ebeling (2010) p.99
} 
For systems with parallel structure $n$ stands for the redundancy grade of the system.

$$
\begin{aligned}
& R_{S}(t)=1-\prod_{i=1}^{n}\left[\operatorname{Pr}\left(T_{i} \leq t\right)\right]=1-\prod_{i=1}^{n} F_{i}(t)=1-\prod_{i=1}^{n}\left[1-R_{i}(t)\right] \\
& R_{S}(t) \geq \operatorname{Max}\left\{R_{i}(t)\right\}
\end{aligned}
$$

Similar to the case of serial systems, the events of failure of individual components are considered to be independent for parallel systems. Other types of system configurations that are just named but not presented in detail here are:

- Mixed systems have both serial and parallel configuration. By introducing partial systems with pure serial or parallel configuration the basic formulas can be applied.

- " $k$-out-of- $n$ " systems have also a parallel configuration. But differently to normal parallel systems one working component is not sufficient. The system is only working properly if at least $k$ of the $n$ components are in operation. An example is a jet that can fly with 2 of the 4 engines still in operation.

- Bridge systems are systems with components that have more than one input or output. Bridge systems can be decomposed to mixed serial/parallel systems.

\subsection{Reliability Growth and Reliability Demonstration Testing}

Reliability growth is defined as the "positive improvement in a reliability parameter over a period of time due to implementation of corrective actions to system design, operation and maintenance procedures, or the associated manufacturing process"

\footnotetext{
${ }^{5}$ see Military Handbook (2009) p.3
} 
In reliability demonstration testing (RDT) an item is exposed to specific tests typically representing the lifetime load profile in order to prove that the system fulfills its reliability specification. Reliability growth management is the management process around planning of the specific tests, the allocation of time and resources to these tests for RDT and the tracking of the reliability in relation to the respective targets.

Typically in the course of a reliability growth program certain system weaknesses and failures are discovered. Subsequently all these failures are subject to corrective actions that are implemented to the system and lead to a higher reliability afterwards.

Reliability growth is achieved with an iterative process consisting of the following steps:

- testing until a certain failure mode occurs

- root cause analysis and definition of countermeasures

- re-design and building of improved parts

- re-testing of the improved parts

Although each item that is newly developed may experience its own specific problems, there are certain models that describe how the exposure to testing time assists in increasing the reliability of the tested item. Many growth models like Duane's model or the AMSAA growth model have been presented. With a larger extent of testing, these models still show an increase in reliability but the respective gradient is decreasing. The growth models typically work with idealized curves for reliability over testing time.

For more details on reliability growth models see Military Handbook (2009) or the literature review of Hall (2008). An overview on reliability tracking models can be found in Ansell, Walls and Quickley (1999). 


\section{General statements on reliability demonstration testing (RDT)}

If RDT is oriented to a specified lifetime target and zero failures occur, the demonstrated reliability can be derived from the binomial distribution. There is a relation between the confidence level $C$, the required reliability $R$ and the sample size $m$. If any two of the three variables are given then the third one is defined with the following relationship ${ }^{6}$ : $C=I-R^{m}$

This means that the required sample size approaches infinity if a high reliability is to be demonstrated with a high confidence level.

Kleyer and Boyle (2004) bring up the following points as in response to wide spread opinions and beliefs around RDT:

- The accuracy of inferring reliability of a large population later in the field derived out of a few weeks RDT can only be limited since important factors like customer usage conditions or temperatures are not considered.

- Even with a high number of data points from testing including failure data and a good fit to the intended reliability function type, the accuracy of predictions for future reliability is in principle limited since it is only based on historic observations and assumptions.

- The later reliability of a system in the field is expected to be at least as high as $R$ from equation (2.22) with the confidence level $C$. But this does not mean that the actual reliability will be as low as $\mathrm{R}$ and the associated warranty cost will really occur.

- Reliability and warranty claims are not only determined by design and production but also by the actual user conditions especially when there is an interaction between the customer and the technical system. These aspects can be hardly considered in RDT but typically reduce the confidence level compared to what was expected based on the test data.

\footnotetext{
${ }^{6}$ see Kleyner and Boyle (2004)
} 


\section{Consequences of random stress levels}

Both the strength of a system and the stress level during operation are subject to variation. High reliability in the field can be achieved if the testing covers the stress level of a $95 \%$ customer, i.e. only $5 \%$ of the customers remain that have duty cycles which lead to a higher stress level for the product.

If RDT under these stress conditions ends with a certain reliability level that is proven, the effective reliability later in the field (taking the actual stress distribution at the different customers into account) will be significantly higher.

A methodology to convert the reliability estimated in testing for the $95 \%$ customer stress level into an expected reliability level in the field is described by Lu and Rudy (2000). They consider three cases: "both stress and strength normally distributed", "both stress and strength log-normally distributed" and "both stress and strength Weibull distributed".

\section{Modeling system reliability growth}

An approach to model the reliability growth of systems was presented by Yadav, Singh and Goel (2003). This methodology assumes a Weibull distribution for all components with a common $\beta$ but individual scale parameters and includes a view of the system from three perspectives: components, functions or failure mechanisms.

The system reliability then can be calculated by multiplying the individual reliabilities of the components (or the functions or the failure modes) which in the end leads to a reliability value for each component concerning each function and failure mode. If a certain failure mode and/or function does not apply to a certain component then the respective matrix element for reliability is equal to $l$. 
Introducing a "criticality index" as a weighing factor for all respective reliability matrix elements of the different components/functions/failure modes makes it possible to estimate the total reliability of the entire system. Yadav et.al. (2003) have proposed that the weighing factors could be derived from the risk metric (RPN) of a failure modes and effects analysis (FMEA). The additional number of systems to be tested can be calculated based on the system reliability target and the estimated system reliability, which in turn is based on the testing conducted so far (prior distribution).

\subsection{Type I and Type II Error in Reliability Demonstration Testing}

The theory of hypothesis testing can be applied to RDT. The experimenter makes a statement on the real value $R$ of the reliability or on certain reliability parameters of a population, called the null hypothesis $\left(H_{0}\right)$, along with an alternative hypothesis $\left(H_{l}\right)$ for the case that the null hypothesis is not true. The statistical inference for $R$ based on the tests is normally related to a reference or target value for reliability $R_{0}$.

In RDT the overachievement of reliability targets is typically not considered to be any kind of a problem therefore $H_{I}$ is formulated as single-sided alternative hypothesis in the following way:

$$
\begin{aligned}
& H_{0}: R=R_{0} \\
& H_{1}: R<R_{0}
\end{aligned}
$$

Using the terminology of hypothesis testing ${ }^{7}$ the following errors can occur when making a decision based on test data or statistics.

- type I error: $H_{0}$ is rejected although it is true - producer's risk

- type II error: fail to reject $H_{0}$ although it is false - consumer's risk

\footnotetext{
${ }^{7}$ see Montgomery and Runger (2003) p. 277 et. seq.
} 


\begin{tabular}{|c|c|c|}
\hline & $H_{0}$ is not rejected & $H_{0}$ is rejected \\
\hline$H_{0}$ is true $\left(R=R_{0}\right)$ & $1-a$ & $a=p($ type I error $)$ \\
\hline$H_{0}$ is false $\left(R<R_{0}\right)$ & $b=p$ (type II error $)$ & $l-b$ \\
\hline
\end{tabular}

Table 2.1 Type I error and Type II error in hypothesis testing

In RDT a type II error is more critical since the real value of the reliability of the population $R_{0}$ is lower than what is stated by $H_{0}$. A type II error is not conservative. Very often the probability of the type II error is called consumer's risk since it corresponds to the erroneous acceptance of a product (e.g. a lot of certain goods) based on a statistical test on a sample ${ }^{8}$. The probability of a type II error will be denoted as $b$.

A statistical acceptance test is based on a mutual test agreement between the consumer and the producer. So the test must consider $a$, the producer's risk that a certain population is rejected based on a test with a sample although the entire population would meet the specified target for reliability $R_{0}$.

Applying the formal procedure of hypothesis testing as proposed by Montgomery and Runger (2003) to RDT leads to:

- a parameter of interest for hypothesis testing: reliability $R$

- a statement on null hypothesis $H_{0}: R=R_{0}$

- a statement on alternative hypothesis $H_{l}: R<R_{0}$

- a choose of significance levels for $a$ (producer's risk) and $b$ (customer's risk)

- an agreement on test procedure and test statistics

- a statement on the rejection region

- the conducting of the tests and computation of the test statistics

- the decision on whether $H_{0}$ should be rejected or not

${ }^{8}$ see Ebeling (2010) p. 344 


\section{Lower Confidence Level (LCL) of reliability}

Related to the theory of hypothesis testing it is also possible to compute a $b$ lower confidence limit (LCL) of the reliability $\left(R_{L C L, b}\right)$ for certain available test data ${ }^{9}$. This means that the probability for wrongly failing to reject the null hypothesis is not greater than $b$ if the real value of the reliability $R$ is lower than $R_{L C L, b}$.

Interpretation of the $b$ LCL of the reliability $R_{L C L, b}$ :

- Given that the real value of reliability is lower than $R_{L C L, b}$, then the probability of ending up with a test statistic that leads to the (incorrect) decision of not rejecting the null hypothesis $H_{0}$ is less or equal to $b$.

- The practical meaning is: When the result of a statistic acceptance test lead to the decision not to reject the null hypothesis $H_{0}$, one can $(1-b)$ confident that the real value of reliability is $R_{L C L, b}$ or greater.

\section{Binomial case}

If $m$ items are tested, each in an independent Bernoulli trial with the reliability $R$, then the probability for at most $x$ failures can be described with the binomial distribution ${ }^{10}$ :

$$
\begin{aligned}
& a=\operatorname{Pr}\{\text { type I error }\}=P\{\text { at most } x \text { failures }\}=\sum_{i=0}^{x}\left(\begin{array}{c}
m \\
i
\end{array}\right) *\left(l-R_{P}\right)^{i} * R_{P}{ }^{m-i} \\
& b=\operatorname{Pr}\{\text { type II error }\}=1-P\{\text { at most } x \text { failures }\}=1-\sum_{i=0}^{x}\left(\begin{array}{c}
m \\
i
\end{array}\right) *\left(l-R_{C}\right)^{i} * R_{C}^{m-i}
\end{aligned}
$$

\footnotetext{
${ }^{9}$ see Montgomery and Runger (2003) p.293

${ }^{10}$ see Montgomery and Runger (2003) p. 273
} 
The producer wants to face a risk not higher than $a$ that the test he has agreed on with the consumer is not considered fulfilled if the reliability is $R_{P}$ or higher. The consumer wants to face a risk not higher than $b$ that the test he has agreed on with the producer is considered fulfilled if the reliability is $R_{C}$ or lower. There are two variables $(x ; m)$ in the two equations of (2.24). This can either be solved by numeric iterations or with the following graphical method.

\section{$\underline{\text { Larson nomogram }}$}

The Larson nomogram allows one to graphically determine the sample size $n$ and the maximum number of failures $x$ for a certain test to make a decision whether a certain lot/product should be accepted or not. Such graphical methods were especially helpful in the years before computers were as common as they are these days.

The Larson nomogram is illustrated in the Figure 2.2. 


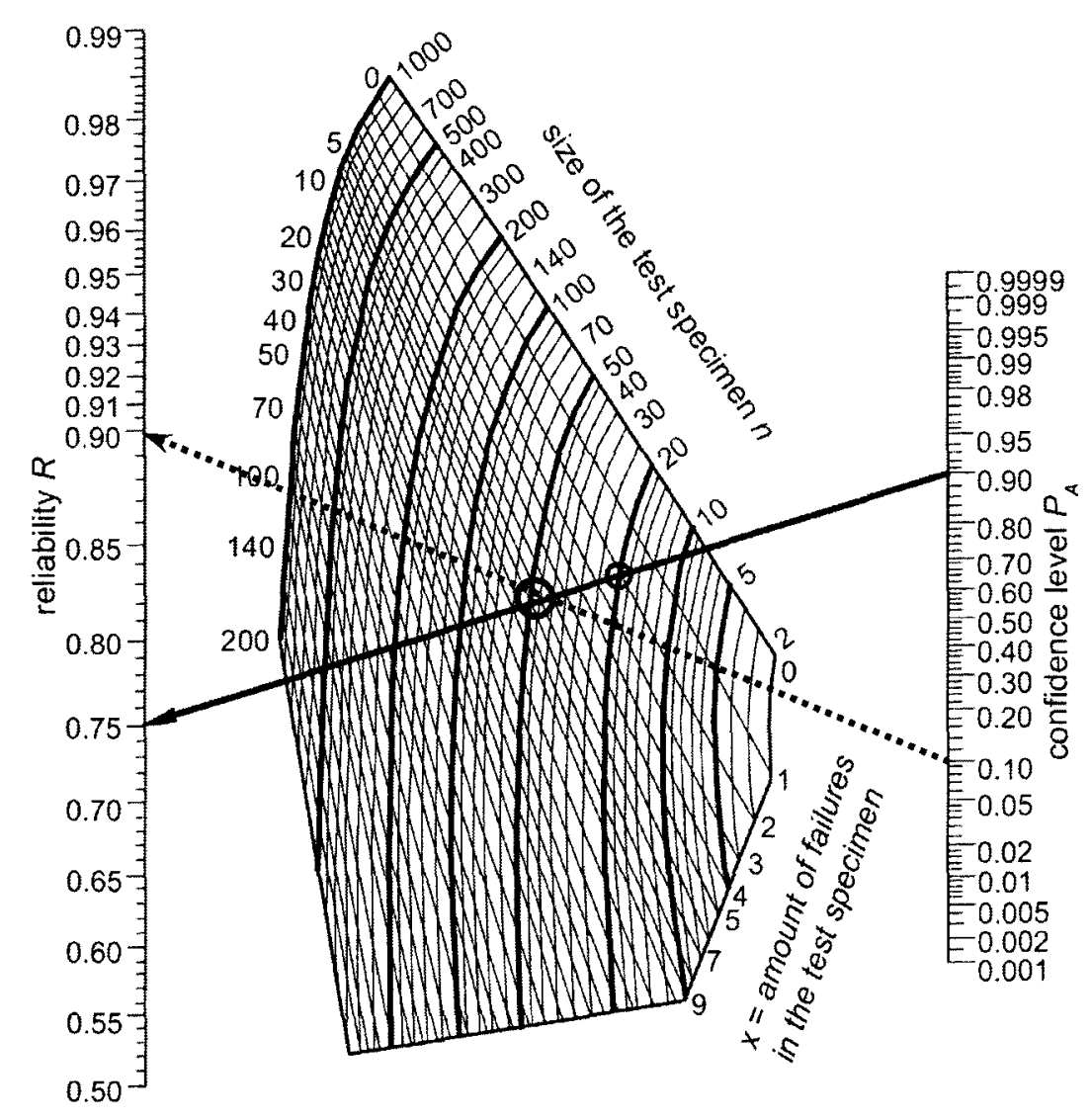

Figure 2.2 Larson nomogram ${ }^{11}$

\section{Example for the application of the Larson nomogram}

The consumer faces the risk of a type II error. If he wants to be sure with a confidence level $b=1-P_{A}=0.10$ that the real value reliability is at least $R=0.75$, any sampling plan on the solid line or above can be accepted:

- no failures permitted in a test of 8 items

- 1 failure permitted in a test of 14 items, and so on

" source: Bertsche (2008) 
The producer carries the risk of a type I error. He can be sure with a confidence level $a=P_{A}=0.90$ that the maximum number of failures in the sample is not exceeded if the real value for is at least $R=0.90$ for all potential testing plans on the dotted line or below:

- up to 1 failure permitted in a test of 5 or less items

- up to 2 failures permitted in a test of 11 or less items, and so on

For any sampling plan left of the intersection a mutual agreement on a test plan could be found that meet both sides' risk willingness:

- up to 6 failures in a test of 40 items

- up to 7 failures in a test of $45 . .47$ parts, and so on

RDT is generally done from the customer's perspective. This means that after successfully conducting the RDT the risk of not exceeding the reliability target should not be higher than the confidence level $b$. But having the reliability above a certain required value is not only of interest for the customer but also for the producer since normally all failures are also associated with cost for the producer for example for warranty.

\subsection{Reliability Function Estimation from Test Data}

There are two common approaches to analyze reliability test data for drawing conclusions on the reliability function and its parameters:

- Estimation of the reliability or the parameters of a reliability function: The estimation can either take place with non-parametric methods or as parameter estimation for a certain underlying reliability function using methods such as MSE or MLE. In both cases, failure data is required. 
- Determination of Lower Confidence Limits (LCL):

Instead of estimating the reliability or the parameters, it is also possible to calculate a LCL, e.g. the value of reliability one can be sure with confidence $(1-b)$ that it is exceeded given the testing that was conducted. LCL is not an estimation of reliability but the limit of a range. The LCL of reliability can be also calculated for situations in which the test data contains zero failures.

Although the focus of the dissertation is on the situation with zero failures both approaches will be briefly presented in this literature review:

\subsubsection{Reliability Estimation Based on Test Data Including Failures}

An estimate for the reliability function or its respective parameters can be made based on different types of test data:

- complete data $=>$ all items are tested until they fail

- type I censored data $=>$ the test is finished for all items that have not failed so far after a fixed test duration

- type II censored data $=>$ the test is finished after a fixed number of failures has occurred

- multiple censored data $=>$ the tests for the various items are stopped after different durations

Testing all items until they fail gives the best statistical information, but this takes a lot of time and requires a large budget. So typically, reliability inference is based on data with at least some items that did not fail, based on censored data. For more information about incomplete data and censoring see Bertsche (2008) (p. 215 et. seq.).

The next paragraphs first cover the non-parametric methods for reliability estimation including reliability of systems and then the estimation of reliability function parameters. 
Non-parametric methods for reliability estimation

There are many different methods to estimate the reliability of a population based on a sample put on test. If there is no prior information on the type of the underlying reliability distribution function non-parametric methods are used. Depending on the type of data (grouped vs. ungrouped or complete vs. censored data) different methods like Mean Rank Estimator, Product Limit Estimator, the Kaplan-Meier Estimator or the Rank Adjustment Method have to be applied. Ebeling (2010) (p. 308 et. seq.) can serve as a starting point to get deeper coverage of non-parametric methods.

All these non-parametric methods and estimators require test data that includes failures. This is not in alignment with the prime focus of this research work which is the inference based on zero-failure data.

Non-parametric system reliability estimation

A non-parametric procedure for the assessment of the reliability for systems with an unknown component reliability function is proposed by Ramírez-Márquez and Jiang (2006). The component test data can be utilized to estimate the component reliability and the variance of this reliability estimation. Basic formulas to estimate the system reliability and its variance when adding an additional component in an either serial or parallel configuration to a subsystem are included. Following this recursive methodology the reliability of a complex system built in a serial-parallel-configuration can be estimated as well as the variance of the reliability estimation by building up the system component by component. Finally they propose to calculate the confidence interval for the real value of system reliability based on Z-statistics. 
Like other non-parametric approaches the described methodology needs a large amount of failure data to be effective. This area falls outside of the focus of this research work which is on reliability statistics based on zero-failure data and typically with small sample sizes.

\section{Estimation of reliability function parameters based on test data}

Knowing the underlying reliability function of an item allows for the calculation of the reliability after a certain operating time $t$ or of the time after which a certain share of the population will still be in operation (e.g. $B_{I 0}$ for $90 \%$ or more still working). For the different types of reliability functions the best fit of the parameters for given test and failure data can be determined with the following methods ${ }^{12}$ :

- Graphical estimation of the parameters by plotting all data points into a probability chart that is adapted to the considered reliability distribution (e.g. normal or Weibull). Using the right probability paper leads to a straight line. This makes the graphical determination of the right parameters very easy.

- With normal regression analyses tools the best fitting curve can also be determined by regression analyses methodology. This method is called Mean Square Estimator (MSE) since the square of the error terms is minimized for the best fitting combination of the reliability function parameters.

- The Maximum Likelihood Estimator (MLE) method finds parameter values that maximize the likelihood function of the existing test data with respect to all parameters of the underlying distribution. The maximum of the likelihood function $L$ can be found by setting all first partial derivatives of $L$ with respect to each parameter equal to zero, and then solving for the parameters.

\footnotetext{
${ }^{12}$ see Ebeling (2010) p. 389 et. seq.
} 
The likelihood function $L$ for a reliability distribution with $k$ parameters $\theta_{l} . . \theta_{k}$ and $m$ parts on test (complete data) with failure time $t_{j}$ is given for:

$\operatorname{Max} L\left(\theta_{l}, \theta_{2}^{\prime}, \ldots, \theta_{k}\right)=\prod_{j=l}^{m} f\left(t_{j} \mid \theta_{l}, \theta_{2}, \ldots, \theta_{k}\right)$

The values of $\theta_{i}$ that maximize equation (2.25) are the maximum likelihood estimators (MLE). These can be found by solving the following set of simultaneous equations:

$\frac{\partial L}{\partial \theta_{i}}=0 \quad$ for all $i=1,2, . . k$

Solving these equations leads to the estimation for the parameters $\hat{\theta}_{l}, \hat{\theta}_{2}, \ldots, \hat{\theta}_{k}$. For more details on the MLE method see Pham (2006) (p. 18 et.seq.).

However, none of these methods (graphical, MSE, MLE) can be applied in the case of zero-failure test data.

\subsubsection{Determination of LCL of Reliability from Data with No Failure}

If there are no failures at all, it is not possible to reasonably estimate the value for reliability parameters or the reliability at a given time. Nelson (1985) explains that the MLE for reliability in the zero failure case is $100 \%$, which is not a realistic estimation. Therefore he proposes to use lower confidence limit (LCL) of the reliability at a certain confidence level $b$ instead. 
The actual reliability of an item is normally unknown but it is not a random variable. The random variable in reliability testing is the number of failures that occur if a sample is exposed to a certain test program. Based on the test durations and considering the fact that there are no failures, the $b$ LCL of reliability can be calculated. The interpretation of $b$ LCL of reliability is, that at this level of reliability (or lower) the probability of having zero failures in the testing is less than $b$. This also means that if the defined test program is repeated very often and the actual reliability is lower than the value that was assumed for the LCL, then at least $(1-b)$ of the repetitions would have one or more failures. On the other hand this means that if no failure occurred one has a really good indication (" $1-b$ confident") that the actual value of the (unknown) reliability is higher than the $b$ LCL.

If the parts are tested exactly for the time to which the statement on reliability is related to (i.e. lifetime testing) then the binomial distribution (2.27) can be applied to calculate the probability of a type II error (e.g. testing $m$ components with $x=0$ failures leads to): $b=\operatorname{Pr}\{$ type II error $\}=1-R^{m}$

This means that the LCL of the real value of the reliability $R$ having conducted $m$ tests with zero failures is:

$$
R_{L C L, b}=(1-b)^{1 / m}
$$

If the parts on test follow a Weibull distribution with shape parameter $\beta$ and the tests have a test time $t_{T}$ different from the reference time $t_{0}$ then $L R=\frac{t_{T}}{t_{0}}$ is the "lifetime ratio" and the reliability is ${ }^{13}$ :

$$
R_{L C L, b}\left(t_{0}\right)=[1-b]^{\frac{t_{0}^{\beta}}{m^{*} t_{T}^{\beta}}}=[1-b]^{\frac{1}{m^{*} L R^{\beta}}}
$$

\footnotetext{
${ }^{13}$ see Bertsche (2008)
} 
A two stage procedure for Weibull components was proposed by Wang and Lu (1992). In a first stage a sample size $m_{l}$ for the chosen confidence level $b$ and the required reliability $R_{\min }$ can be calculated.

$$
m_{1}=-\frac{\chi_{b ; 2}^{2}}{2 * L R^{\beta} * \ln \left[R_{\min }\left(t_{0}\right)\right]}
$$

If no failure has occurred after testing $m_{l}$ parts the product is accepted.

If one failure has occurred a second stage of testing with $m_{2}$ parts is conducted. Wang and Lu propose to accept the lot if no additional failure has happened within the next stage:

$$
m_{2}=M-m_{1}=\frac{\chi_{b ; 2}^{2}}{2 * L R^{\beta} * \ln \left[R_{\min }\left(t_{0}\right)\right]}-\frac{\chi_{b ; 4}^{2}}{2 * L R^{\beta} * \ln \left[R_{\min }\left(t_{0}\right)\right]}
$$

Lower Confidence Limit (LCL) of reliability with few or no failures for Weibull

Nelson (1985) proposed a method for the Weibull distribution with given $\beta$ and $r$ failures to calculate the $b$ LCL of reliability $R_{L C L, b}$, the scale factor $\theta_{L C L, b}$ and the lifetime $B_{X L C L, b}$. The $b \mathrm{LCL}$ of the scale factor is:

$$
\theta_{L C L, b}=\left(\frac{2 \sum_{j=1}^{m} t_{j}^{\beta}}{\chi_{b ; 2 r+2}^{2}}\right)^{I / \beta}
$$

This can be substituted into the Weibull equations (2.11) and (2.16) for reliability $R_{0}$ and lifetime $B_{X}$ :

$R_{L C L, b}\left(t_{0}\right)=e^{-\left(\frac{t_{0}}{\theta_{L C L, b}}\right)^{\beta}}=e^{-\left(\frac{t_{0}^{\beta *} \chi_{b ; 2 r+2}^{2}}{2 \sum_{j=1}^{m} t_{j}^{\beta}}\right)}$ 


$$
B_{x, L C L, b}=\theta_{L C L, b} *\left[-\ln \left(1-\frac{x}{100 \%}\right)\right]^{1 / \beta}=\left(\frac{-2 * \ln \left(1-\frac{x}{100 \%}\right) * \sum_{j=1}^{m} t_{j}^{\beta}}{\chi_{\text {conf } ; 2 r+2}^{2}}\right)^{1 / \beta}
$$

Since the LCL of the reliability for typical values of $b=0.05 \ldots 0.30$ is very low Nelson (1985) has proposed to substitute in $b=0.50$ for a more realistic expected value. But there is no further statistical argumentation for this approach.

The methodology by Nelson (1985) described above was further refined in different ways:

- Ke (1999) and Wang (1991) have provided tables for specific combinations of reliability and confidence level.

- The estimation of the Weibull parameter $\beta$ based on historic experience can be wrong. Huang and Porter (1991), Huang (1997) as well as Lu and Wang (2008) claimed that there is a single value $\beta_{0}$ which leads to a minimum reliability. So the methodology can be applied even if $\beta$ is unknown. On the other hand a sensitivity analyses could be conducted to evaluate the maximum error of wrongly guessing $\beta$. The value of the worst case $\beta_{0}$ can be iteratively determined by the Newton-Raphson method.

- If the testing in the laboratory is much tougher than the usage by the customer later in the field, it is sufficient that a lower reliability level is demonstrated compared to the reliability expected later in the field. Based on the approach above Lu and Rudy (2001) show how to quantify this effect in order to reduce the required number of samples on test and/or the test duration.

- Allmen and Lu (1993) compare two objectives or approaches to RDT. The one objective is to detect a certain non-conformity in testing that would occur with a specific rate afterwards. The other objective is the demonstration test to verify the required reliability at a certain confidence level. 
Different to that there are also methods presented for one-shot-devices. Bailey (1997) has listed six different reliability estimators for one-shot-devices and ordered them according to how conservative these estimators are. The reliability of one-shot-devices does not follow the Weibull distribution since it is not dependent on lifetime $t$. Each shot is an independent Bernoulli trial.

The methods presented so far in this section are suitable for situations in which the parts are either functioning or are instantaneously failing. The actual degradation of the system is not known to the test engineer. But Yang (2009) introduces a method called Degradation Bogey Testing where there is a certain metric that describes the status of degradation of the system prior to the occurrence of a failure. The system is functioning as long as the degradation metric is below a certain threshold. Based on the actual test results, meaning the measurement of the degradation metric at the different test times, the distribution of degradation at a specific operation time can be estimated.

Kim and Yum (2009) have introduced a method for unknown Weibull parameters. It is applicable for accelerated testing with known acceleration factor and type I censored data. A certain lot is accepted if no failure in testing occurs. If there are failures then the respective test statistic and the acceptance criteria are iteratively determined.

If there is certain information available on the reliability of e.g. a previous product then the shrinkage estimation methods of Baklizi and Ahmed (2008) and Jiang, Lim, Zuo and Guo (2010) consider both the reliability estimation of the current product and the reliability of the historic product. The shrinkage estimator is between these two reliability values. Whereas the first ones Baklizi and Ahmed (2008) do not explain how set the value of the shrinkage factor there is a procedure presented by the latter ones to calculate the shrinkage factor with a so called dissimilarity index. 


\subsection{Influence of RDT on Product Life Cycle Cost}

It is intuitive that more reliability growth testing will increase the probability that most issues of a new product will be discovered prior to its launch (otherwise the reliability growth testing would not be effective). Therefore the associated warranty cost of a scenario with intensive testing will be lower compared to a scenario with less testing. A model for minimization of the product cost from a buyer's perspective that includes design cost, validation cost, manufacturing cost, warranty cost and seller's profit was presented by Kleyner, Sandborn and Boyle (2004) and Kleyner and Sandborn (2008):

$$
\begin{array}{r}
\text { Design Cost } \\
+ \text { Validation Cost } \\
+ \text { Manufacturing Cost } \\
+ \text { Warranty Cost } \\
+ \text { Seller's Profit } \\
\hline \text { Buyer's cost }
\end{array}
$$

The part of the total cost that can be controlled by validation is indicated by writing in italics in the equation (2.35) above. It is the sum of the validation cost (dependent e.g. of the fixed cost per test unit and hourly costs for testing on rigs and/or personnel) and of the warranty cost. The latter depends on the warranty period, the repair cost of a failed unit and the share of units failed as difference from the reliability to $100 \%$.

Figure 2.3 shows the theoretical relation between warranty cost and development cost i.e. design plus validation cost. The total cost is $\mathrm{U}$-shape and there is a certain reliability level or extent of validation testing that leads to the minimum of the total cost. 


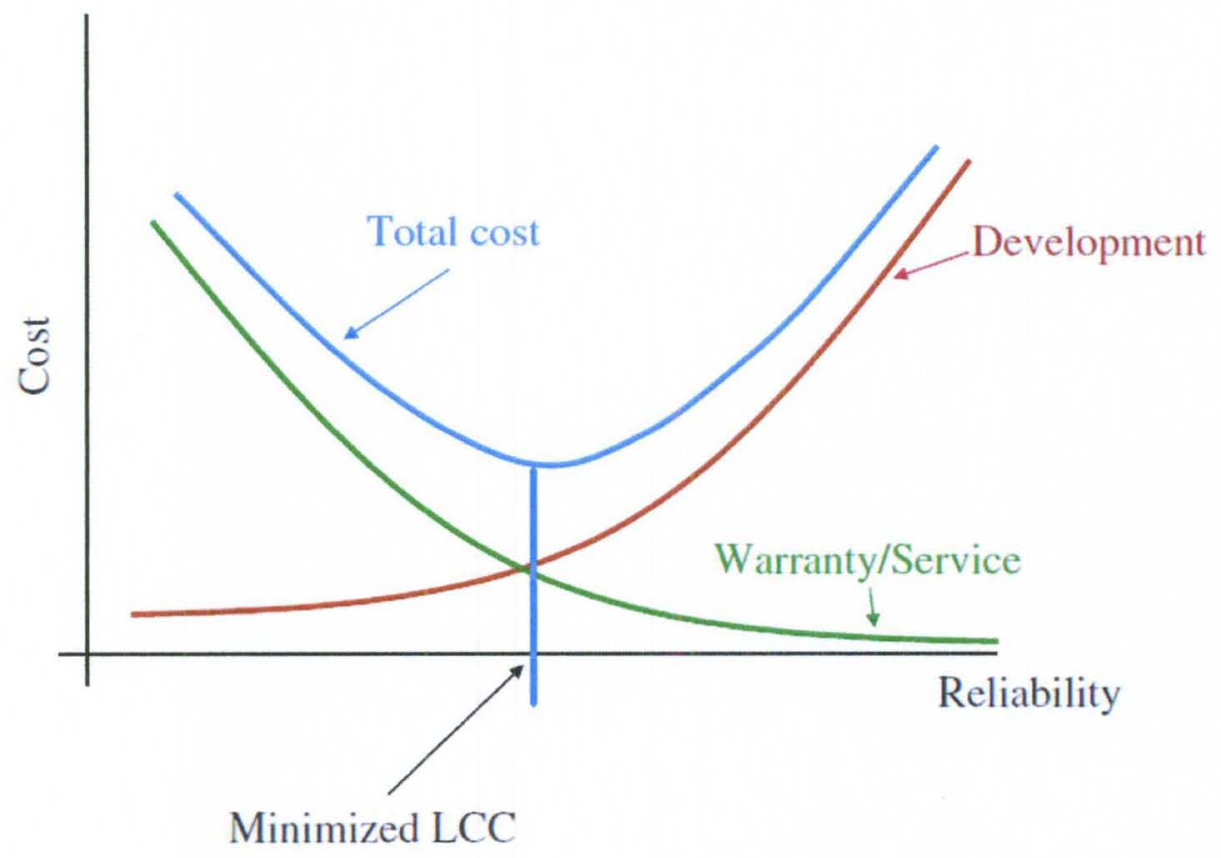

Figure 2.3 Theoretical product development cost versus reliability curve $\mathrm{e}^{14}$

Typically the term for warranty is subject to uncertainty. But it is the purpose of RDT and reliability growth to reduce the failure rate in the field and to reduce the induced warranty cost. In order to optimize the entire cost controllable by validation it is necessary to make assumptions on the relationship of the actual reliability to the extent of the accomplished test program.

Optimizing the RDT programs to meet reliability requirements in the most efficient way will be addressed in this work.

${ }^{14}$ source: Kleyner and Sandborn (2008) 


\subsection{Accelerated Lifetime Tests (ALT)}

One aspect of this dissertation is the use of Accelerated Lifetime Testing (ALT). This section provides a brief description of this topic.

If the lifetime of a certain product and the expectation of its reliability are very high then the RDT will take a lot of time and consume a large budget. But if the testing of the product takes place at a higher than nominal load then the test duration can be shortened significantly. ALT is the process of testing at a higher than normal stress level to simulate the entire product life in a shorter time with increased load.

To quantify the acceleration factor, a deep understanding of the physics of failure is required, especially of how the increased load reduces the lifetime or the wear-out failure period. This will vary strongly if the damaging mechanism is amplified by wear, temperature, pressure or mechanical stress. The Arrhenius model is a very frequently used model in the case where the influence of temperature is to be considered. As an alternative it is also possible to do lifetime tests at different stress levels and to determine the acceleration factor by regression analyses.

If the acceleration factor is overestimated the inference of RDT on product reliability will be too optimistic. 


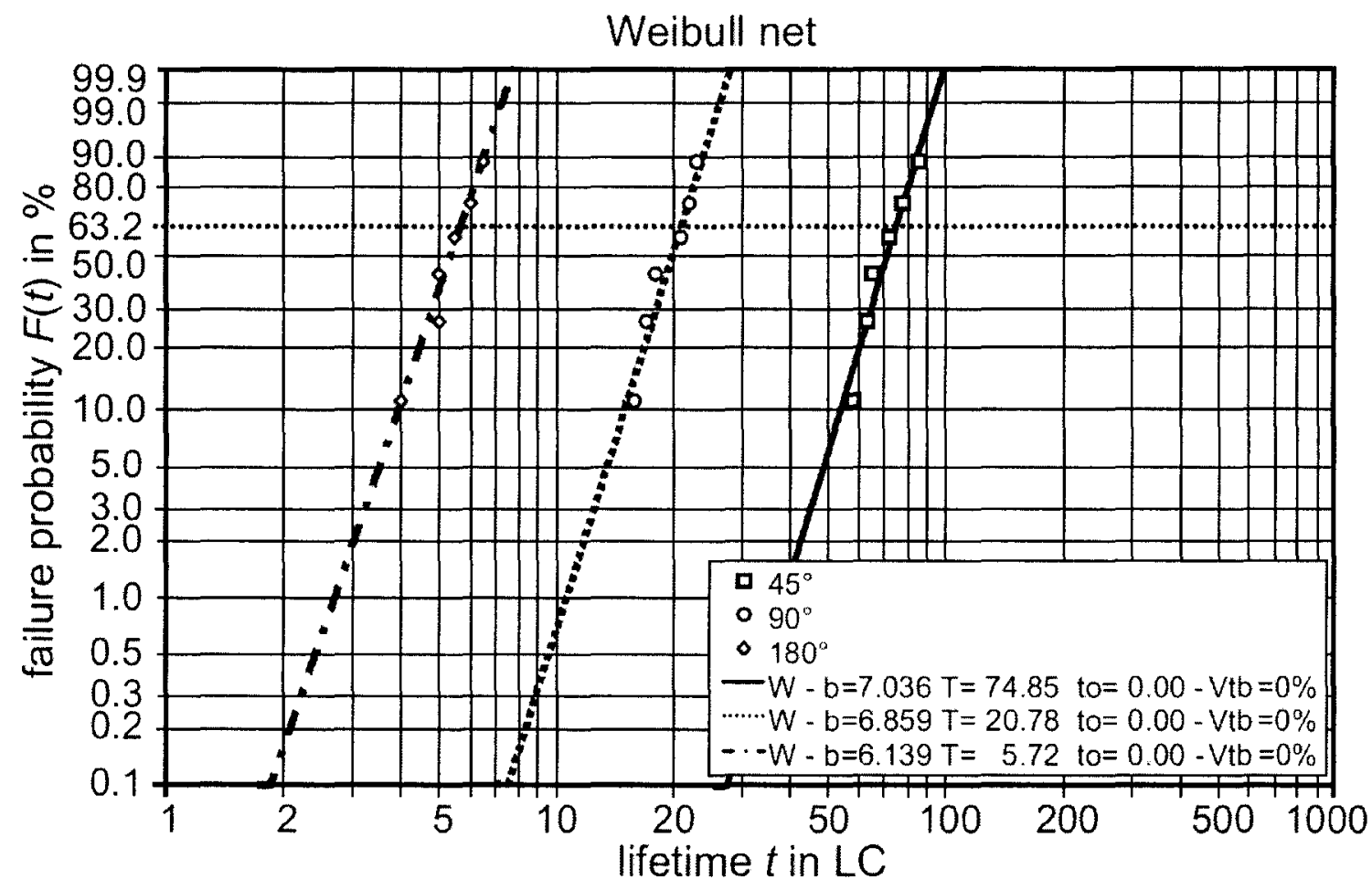

Figure 2.4 ALT - failure distribution (paperclip bending test with diff. angles) $)^{15}$

ALT with Weibull components at different load levels result in failure distributions on parallel lines in the Weibull diagram (see Figure 2.4). If the lines are not parallel it means that the failure mechanisms at the different load levels are not identical and the system is not tested with an adequate acceleration.

In the example above paperclips were bent with different bending angles. The straight regression lines in the Weibull chart are parallel; this means that the Weibull parameter $\beta$ is identical for each bending angle and it is possible to calculate an acceleration factor $A F$ between two different bending angles. If $45^{\circ}$ is reflecting the nominal load case than an acceleration by more than $A F=10$ can be achieved by bending up to $180^{\circ}$.

${ }^{15}$ source: Bertsche (2008) 
If systems with various components are tested, it is very often not possible to accelerate the testing for all components with the same acceleration factor $A F$. Some components can be ALT-tested by increasing the mechanical load or the vibration level. Other components suffer more from higher ambient temperature or exposure to a corrosive atmosphere. At the end of RDT the outcome may be a test protocol documenting that the various components were exposed to different effective test durations.

The literature on ALT models is extensive. This dissertation is not presenting a new ALT model but will consider the impact of ALT coinciding with the different effective testing durations for each component due to the different acceleration factors per test. For more on ALT, see Elsayed (2003).

\subsection{Confidence Limits on System Reliability}

An overview of methods to calculate the lower confidence limit of reliability of a system based on component reliability data existing at that time was given by O'Neill (1972). He has listed a total of 14 methods. None of them is specifically dedicated to Weibull components. Some of them just multiply the component LCL of reliability which leads to a very conservative and pessimistic LCL of system reliability.

Tian (2002) reviewed the existing literature on LCL of system reliability by considering more than 30 references. There are methods mentioned for the estimation of LCL as well as for its exact calculation. Most of the methods require data with observed failures. Tian (2002) states that there is no analytical method applicable for the LCL calculation of systems with Weibull components and suggests therefore the utilization of Monte Carlo Simulation (MCS). 
A method to calculate the LCL of reliability of a system with Weibull components was presented by Klyatis, Teskin and Fulton (2000). This method is not applicable for zero failure but works with complete and censored data. It is intended for situations in which the different components of the system were tested individually with different sample sizes and test durations. Based on the rate of censoring different factors have to be determined table-based. They are used to calculate a LCL of the reliability of the components and finally the system.

\subsection{Critique}

There is a large amount of literature for certain aspects of reliability engineering like reliability growth models or ALT. Many of the methods for the estimation of the reliability are only suitable when extensive test and failure data is available. Although the testing effort and the respective budgets in many industries (like the automotive industry) are comparatively large, the actual sample sizes and achieved mileages prior to product launch are often too low for these methods. Therefore instead of estimating the reliability, a LCL of reliability has to be calculated.

There is no adequate method known that allows for the determination of the LCL of the system reliability if the system consists of Weibull components and the reliability demonstration testing has experienced no failures.

Such a method could be integrated into a framework for optimizing an actual RDT program to meet certain target values for LCL of reliability or $B_{X}$-lifetime in the most efficient way. 


\section{FRAMEWORK FOR SYSTEM RELIABILITY ESTIMATION WITH ZERO-FAILURE DATA}

At the beginning of this chapter, important definitions to be used in the proposed framework are introduced. Next the underlying premises and assumptions are clarified. Then theorems of the LCL of system reliability and the system $B_{X}$-lifetime are presented. Finally the methodology is illustrated with an example at the end of the chapter.

\subsection{Definitions}

The proposed methodology is only applicable when reliability testing is conducted with zero failures.

Let $i$ denote a component $(i=1 . . n)$ and $j$ a test $(j=1 . . m)$. The respective test time of test $j$ is $t_{j}$. The acceleration factor $A F_{i j}$ for component $i$ in test $j$ is related to the nominal load under normal operating conditions. The Boolean variable $o_{i j}\left(o_{i j}=[0,1]\right)$ indicates the relevance of the outcome of test $j$ for component $i ; o_{i j}=1$ means that test $j$ was successful for component $i$ and the respective test time can be used in the computation of the reliability level whereas $o_{i j}=0$ means that this test result does not impact component $i$.

If single components, instead of entire systems, were tested, then $t_{i}$ is the test duration for component $i$ and $m_{i}$ is the number of components on test. This assumes that there exists only one type of test per component. 
Probability of zero failures in multiple test runs

The reliability of a Weibull item is defined according to equation (2.10). If a sample of $m$ items is tested in independent trials with duration $t_{j}(j=1,2, \ldots m)$ the probability of zeros failures in all $m$ tests is the product of the reliability at the individual test durations:

$$
\operatorname{Pr}(\text { Ofailures })=e^{-\left(\frac{t_{I}}{\theta}\right)^{\beta}} * \ldots * e^{-\left(\frac{t_{m}}{\theta}\right)^{\beta}}=\prod_{j=1}^{m} e^{-\left(\frac{t_{j}}{\theta}\right)^{\beta}}=e^{-\frac{\sum_{j=1}^{m}\left(t_{j}^{\beta}\right)}{\theta^{\beta}}}
$$

Weibull equivalent single test duration

As a shortcut, the Weibull equivalent single test duration $T W$ for an item with Weibull parameter $\beta$ and $m$ tests is introduced:

$T W=\beta \sqrt{\sum_{j=1}^{m}\left({ }_{t}^{\beta}{ }_{j}\right)}$

The Weibull equivalent single test duration, $T W$, represents the accumulation of all test durations for a given item. The reliability at TW is equivalent to the probability of having zero failures in all $m$ tests.

The dimension of TW is identical to the dimensions of the test durations $t_{j}$. It can either be based on time (hours) or on usage, such as mileage. It is obvious that for a certain set of test durations $t_{j}$ the value of $T W$ depends on the Weibull parameter $\beta$. For the exponential case $(\beta=1) T W$ is just the sum of all test durations.

With the definition of (3.2), the equation (3.1) for the probability of zero failures in $m$ tests can be simplified to: 
$\operatorname{Pr}($ O failures $)=e^{-\frac{T W^{\beta}}{\theta^{\beta}}}$

If a system is considered instead of a single item, and taking the definitions of acceleration factor $A F_{i j}$ and the variable $o_{i j}$ into account, the Weibull single test duration for component $i(i=1 . . n)$ is defined as follows:

$T W_{i}=\beta_{i} \sqrt{\sum_{j=1}^{m}\left(o_{i j} * A F_{i j} * t_{j}\right)^{\beta_{i}}}$

In equation (3.4) the actual duration of the tests are identical for all components. But since the acceleration factors $A F_{i j}$ can be different per test and per component, the contribution of each test to reliability demonstration of the components can also be different.

If each component $i$ of the system is tested with an individual test procedure with duration $t_{i}$ and a sample size of $m_{i}$ components, the Weibull equivalent single test duration $T W_{i}$ for this component $i$ is:

$T W_{i}=\sqrt[\beta_{i}]{t_{i}^{\beta_{i} * m_{i}}}=t_{i} * \beta_{i} m_{i}$

The introduction of Weibull equivalent single test duration $T W_{i}$ is a shortcut to keep subsequent equations shorter. For the frequent case of $\beta>1$ the longer test durations are weighted more in the calculation of $T W_{i}$ compared to the simple addition of the test durations.

For the Weibull factor $\beta=1$ (i.e. exponential distribution) $T W_{i}$ is identical to the sum of test durations. If there are components with different $\beta_{i}$ then the Weibull equivalent single test durations $T W_{i}$ will be different for each component even when all test durations are the same. Table 3.1 gives a numerical example to illustrate the usage of $T W_{i}$ for a specific component with the Weibull shape parameter $\beta_{i}=1.5$ : 


\begin{tabular}{|c|c|c|}
\hline & $t_{i}$ & $t_{i}^{\beta_{i}}$ \\
\hline & 1000 & 31622.8 \\
\hline & 1500 & 58094.8 \\
\hline & 1250 & 44194.2 \\
\hline & 800 & 22627.4 \\
\hline$\sum$ & 2000 & 89442.7 \\
\hline & 2550 & 245981.8 \\
\hline & 6550 & $\begin{array}{l}T W_{i}= \\
3925.9\end{array}$ \\
\hline
\end{tabular}

Table 3.1 Example to illustrate $T T_{i}$ and $T W_{i}$ for $\beta_{i}=1.5$

The Weibull equivalent single test duration $T W_{i}$ is the equivalent test duration of a single test that leads to the same probability of zero failures (i.e. reliability) as the time to run all $m$ tests together (on component $i$ ).

Simplifications of the LCL of single item reliability for the case of zero failures

For the zero-failure case $(r=0)$, equations (2.32) to (2.34) for the LCL of shape parameter, LCL of reliability and LCL of $B_{X}$ - lifetime can be further simplified by using equation (App. 2.4) from Appendix 2 with $\chi_{b ; 2}^{2}=2 \ln (1 / b)$ :

$\theta_{L C L, b}=\left(\frac{\sum_{j=1}^{m} t_{j}^{\beta}}{\ln (1 / b)}\right)^{1 / \beta}$ 
$R_{L C L, b}\left(t_{0}\right)=e^{-\left(\frac{t_{0}^{\beta} \ln (1 / b)}{\sum_{j=1}^{m} t_{j}^{\beta}}\right)}=e^{\left(\frac{t_{0}^{\beta} * \ln (b)}{\sum_{j=1}^{m} t_{j}^{\beta}}\right)}$

$$
B_{x, L C L, b}=\left(-\frac{\ln \left(1-\frac{x}{100 \%}\right) * \sum_{j=1}^{m} t_{j}^{\beta}}{\ln (1 / b)}\right)^{1 / \beta}=\left(\frac{\ln \left(1-\frac{x}{100 \%}\right) * \sum_{j=1}^{m} t_{j}^{\beta}}{\ln (b)}\right)^{1 / \beta}
$$

Substituting the Weibull equivalent single test duration $T W_{i}$ from (3.4) or (3.5) into equation (3.7) leads to the LCL of reliability of component $i, R_{C i ; L C L ; b ; i}$. Applying basic rules for exponentiation ${ }^{16}$ allows to further simplify:

$R_{C i ; L C L, b}\left(t_{0}\right)=e^{\left(\frac{t_{0}^{\beta_{i *} \ln (b)}}{T W_{i}^{\beta_{i}}}\right)}=b^{\left(\frac{t_{0}^{\beta_{i}}}{T W_{i}^{\beta_{i}}}\right)}$

A mathematical proof of equation (3.9) for single items can be found in Appendix 4.

$R_{C i ; L C L, b}\left(t_{0}\right)=b^{\left(\frac{t_{0}^{\beta_{i}}}{\sum_{j=1}^{m}\left(o_{i j}{ }^{*} A F_{i j}{ }^{*} t_{j}\right)^{\beta_{i}}}\right)}=b^{\left(\frac{t_{0}^{\beta_{i}}}{t_{i}^{\beta_{i} m_{i}}}\right)}$

${ }^{16}$ basic rules for exponentiation: $e^{\ln (b)}=b$ and $a^{\left(b^{*} c\right)}=\left(a^{b}\right)^{c}$ 
The respective LCL of $B_{X}$-lifetime $B_{X ; C i ; L C L ; b}$ of the component $i$ is:

$$
\begin{aligned}
& B_{X ; C i ; L C L ; b}=\left(\frac{\ln \left(1-\frac{x}{100 \%}\right) * T W_{i}^{\beta_{i}}}{\ln (b)}\right)^{1 / \beta_{i}} \\
& B_{X ; C i ; L C L ; b}=\left(\frac{\ln \left(1-\frac{x}{100 \%}\right) * \sum_{j=1}^{m}\left(o_{i j} * A F_{i j} * t_{j}\right)^{\beta_{i}}}{\ln (b)}\right)^{1 / \beta_{i}} \\
& B_{X ; C i ; L C L ; b}=\left(\frac{\ln \left(1-\frac{x}{100 \%}\right) * t_{i}^{\beta_{i} * m_{i}}}{\ln (b)}\right)^{1 / \beta_{i}}
\end{aligned}
$$

\subsection{Assumptions and Premises}

The proposed methodology is based on the following underlying assumptions:

1. Zero failures have occurred during testing:

This means that either no issues have arisen or that all failures are resolved through design or process changes.

2. All components follow a Weibull distribution with known shape parameter $\beta_{i}$ : $\beta_{i}$ is known from analysis of historical systems or can be estimated by experts. The components are arranged in a serial configuration. The failure behavior of all components is assumed to be mutually independent. 
3. Countermeasures for issues do not affect other components:

If an issue arises there will be a countermeasure introduced that only focuses on the failing component. We assume that the design changes do not have an influence on any other component.

4. The acceleration factors $A F_{i j}$ for the different tests and components are known.

5. If a sub-system of the system is a copy from another (already tested) system then the respective test durations $\left(T W_{i}\right)$ for this respective sub-system can be converted to the considered system.

6. Failures with a prototype specific root cause will be ignored:

- Countermeasures will be focused on prototype specific processes only and do not lead to a design change.

- The test time considered for this specific test and the component affected by the prototype specific failure will be zero (i.e. $o_{i j}=0$ ).

- The test time for components not affected by the failure can be calculated by considering the respective acceleration factors as if there has not been a failure.

7. If the design of a component has to be modified for implementing a countermeasure then the testing times of the respective component for tests conducted before implementation of the modification will be ignored.

8. The design level of the system that will be finally released had no failures during RDT since all issues occurred were fixed with countermeasures. 
Premise 7 is conservative since modifications or countermeasures normally do not result in a total change of concept for a component, but rather are a kind of evolutionary improvement steps. Therefore, a certain part of the validation could also be applied to the modified system, i.e. the effective validation time after implementing the countermeasure could be higher than zero.

On the other hand premise 8 is optimistic since this assumption means that all issues that occur will be fixed effectively. In reality not all implemented countermeasures will fix the issues on the first try.

Taking the previously mentioned premise 7 into account, the combined risk of premise 7 and 8 seems to be adequate. If there is a big concern that the countermeasure will not be effective there should be some pretesting prior to the restart of RDT with the modified component.

\subsection{First Theorem - LCL of Reliability of a System with Zero-Failure Data}

This section presents a theorem for the lower confidence limit of system reliability. This theorem is the basis for the framework for determination of the system reliability and for subsequent models for sensitivity analyses and optimization. An example will follow in the next section.

A Weibull component $i$ with shape parameter $\beta_{i}$ that is exposed to the Weibull equivalent single test duration $T W_{i}$ (as defined in equations (3.4) or (3.5)) with zero failures occurring has the following $b \mathrm{LCL}$ of component reliability $R_{C i ; L C L ; b}$ at duration $t_{0}$ according to (3.8): 
$R_{C i ; L C L ; b}\left(t_{0}\right)=e^{\left(\frac{t_{0}^{\beta_{i} * \ln (b)}}{T W_{i}^{\beta_{i}}}\right)}=b^{\left(\frac{t_{0}^{\beta_{i}}}{T W_{i}^{\beta_{i}}}\right)}$

Theorem:

A system consisting of $n$ Weibull components with shape parameter $\beta_{i}$ that is exposed to the Weibull equivalent single test duration $T W_{i}$ (as defined in equations (3.3) or (3.4)) with zero failures occurring has the following $b \mathrm{LCL}$ of system reliability $R_{S ; L C L ; b}$ at duration $t_{0}$ :

$$
R_{S ; L C L ; b}\left(t_{0}\right)=\operatorname{Min}_{i} R_{C i ; L C L ; b}\left(t_{0}\right)=\operatorname{Min}_{i}\left[b^{\left(\frac{t_{0}^{\beta_{i}}}{T W_{i}^{\beta_{i}}}\right)}\right]
$$

The equation above means that the LCL of system reliability is identical to the lowest LCL of component reliability calculated according to equation (3.14).

Depending on the different parameters $\beta_{i}$ the specific component being the limiting one for the calculation of $R_{S ; L C L ; b}$ can change at different points in time $t$. The confidence level $b$ is equal to the probability of a type II error. This failure type occurs if the real value for system reliability is lower than what was calculated as LCL of the system reliability. Given the situation that the real value for reliability is lower than $R_{S ; L C L ; b}$ then the probability of finishing RDT without any failures must be less or equal than $b$. This means that one can be sure with probability $(l-b)$ that the real value for system reliability is higher than $R_{S ; L C L ; b}$.

Based on pre-investigations there is an indication that the theorem (3.15) leads to a conservative value for the LCL of system reliability $R_{S ; L C L ; b}$. 
In the course of the dissertation (see Chapter 4) a mathematical proof will be presented that the theorem in equation (3.13) for the LCL of the system reliability $R_{S ; L C L ; b}$ is conservative.

For further explanation, the special case with $n=2$ identical components can be found in Appendix 2.

\subsection{Second Theorem - LCL of $B_{X}$ - lifetime of a System with Zero-Failure Data}

This section proposes a theorem for the lower confidence limit of the $B_{x}$ - lifetime. An example will follow in the next section.

A Weibull component $i$ with shape parameter $\beta_{i}$ that is exposed to the Weibull equivalent single test duration $T W_{i}$ (as defined in equations (3.4) or (3.5)) with zero failures occurring has the following $b \mathrm{LCL}$ of component $B_{X}$ - lifetime $B_{X ; C i ; L C L ; b}$ according to (3.11):

$B_{X ; C i ; L C L ; b}=\left(\frac{\ln \left(1-\frac{X}{100 \%}\right) * T W_{i}^{\beta_{i}}}{\ln (b)}\right)^{1 / \beta_{i}}$

Theorem:

A system consisting of $n$ Weibull components with shape parameter $\beta_{i}$ that is exposed to the Weibull equivalent single test duration $T W_{i}$ (as defined in equations (3.4) or (3.5)) with zero failures occurring has the following $b$ LCL of $B_{X}$-lifetime of the system $B_{X ; S ; L C L ; b}$ : 


$$
\begin{gathered}
B_{X ; S ; L C L ; b}=\underset{i}{\operatorname{Min}}\left[B_{X ; C i ; L C L ; b}\right] \\
B_{X ; S ; L C L ; b}=\operatorname{Min}\left[\left(\frac{\ln \left(1-\frac{X}{100 \%}\right) * T W_{i}^{\beta_{i}}}{\ln (b)}\right)^{1 / \beta_{i}}\right]
\end{gathered}
$$

Equation (3.17) means that the LCL of $B_{X}$ - lifetime is identical to the lowest LCL of component $B_{X}$-lifetime calculated according to equation (3.14).

Depending on the different parameters $\beta_{i}$ the specific component being the limiting one for the calculation of $B_{X ; S ; L C L ; b}$ can change for a different share $X$ of permitted systems that have failed.

LCL of $B_{X}$ - lifetime means:

Given the situation that the real value for system $B_{X}$ - lifetime is lower than $B_{X ; S ; L C L ; b}$, then the probability of finishing RDT without any failures is lower than $b$ (see Chapter 2.4). This means that one can be sure with probability $(1-b)$ that the real value for system $B_{X}$-lifetime is not lower than $B_{X ; S ; L C L ; b}$.

The proof of the theorem of the system $B_{X}$-lifetime in equation (3.17) will take place, together with the proof of the theorem of the system reliability $R_{S ; L C L ; b}$ according to equation (3.15), in Chapter 4. Before presenting those proofs, it is helpful to illustrate the theorems with a numerical example. 


\subsection{Example \& Background}

The following example is provided to illustrate the application of the theorems. It considers the validation of exhaust aftertreatment systems for trucks. The main purpose of these systems is the reduction of exhaust emission from truck diesel engines.

Soot particles are a major type of pollutant that must be removed by the exhaust aftertreatment system. They are collected in the particulate filter and burned there. Another important type of pollutants is nitrogen oxide $\mathrm{NO}_{\mathrm{X}}$ that is reduced to pure nitrogen and water on a SCR-cat. (selective catalyst reduction) by consuming aqueous urea solution. Both pollutants are limited by emission legislation.

The test engineer designs the validation program that must cover all components within the system. The tests take place on test benches and in durability test vehicles. The test engineer must have information on how to transform the test bench time into the equivalent vehicle mileage and on how to determine the specific acceleration or load factors for each component in each test cycle. The Weibull factor $\beta_{i}$ for each component is known from past data. Normally there is no single test procedure that allows for the same high acceleration factor on all components.

There are tests that basically help to validate the entire system whereas others are mainly focused on specific components. 


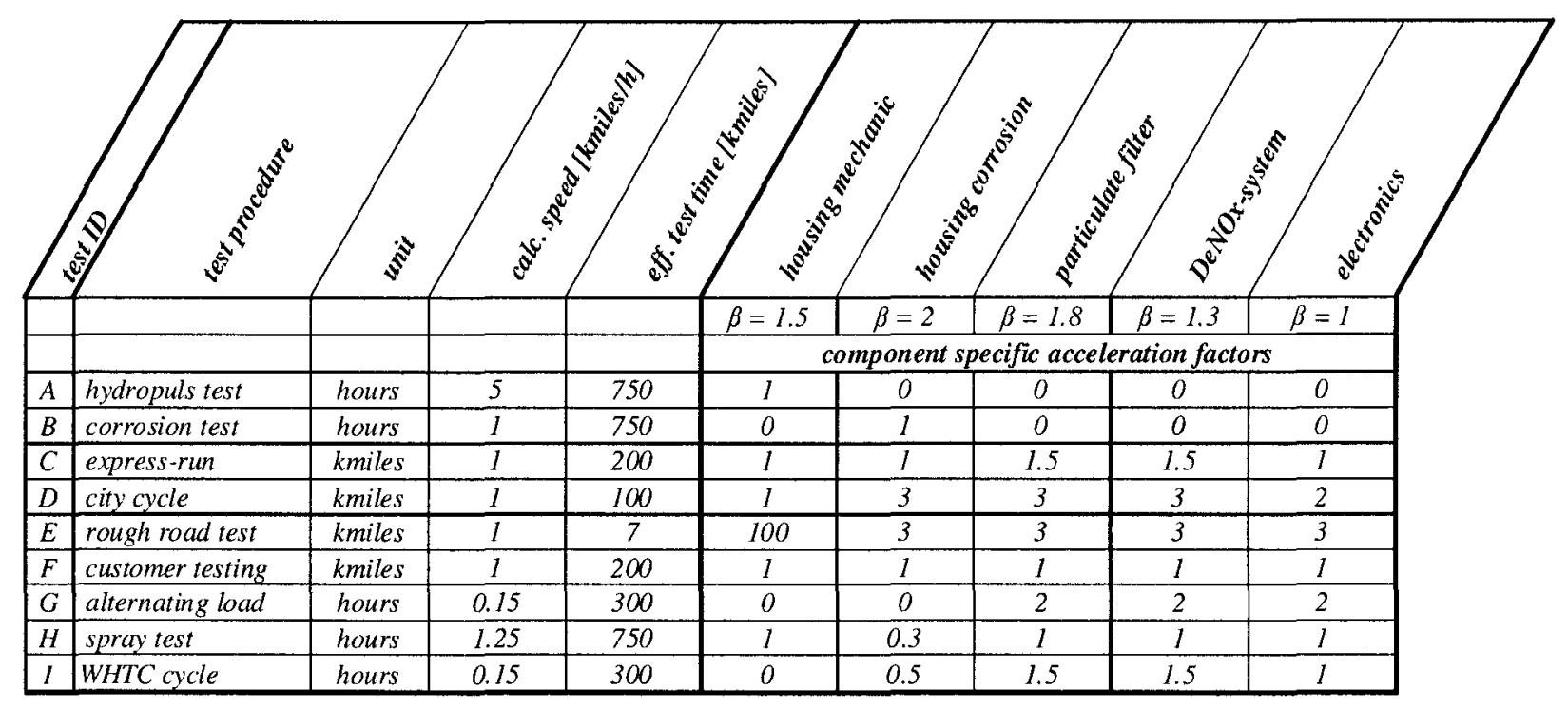

Table 3.2 Test Procedure Database

The documentation of the validation after conducting the RDT includes information on the test procedure and duration/mileage, as well as on the results. If a specific component fails, the test duration will not be taken into account completely when it comes to calculation of the demonstrated level of reliability. If there is change to a component (as a countermeasure to an observed issue) then all tests durations with the old design level for this component cannot be counted.

On any failure that occurs during testing, a decision has to be made on how much and which test times have to be ignored, which depends on the root cause and the kind of countermeasure.

The RDT documentation in the Table 3.3 belongs to the example from the context above and shows the available information after finishing the test program including durations and results. In this table, all test durations are transferred into the unit kmiles (1000 miles) based on actual duration and acceleration factor. 
If there was an incident or damage in the test it is indicated in the comment column and by a bracket [ ]. When the countermeasure has required a design change on a certain component then the respective test times prior to that change must not be considered and are put into brackets [ ] as well.

The bottom line shows the total effective mileage/duration that each component was exposed to during the entire validation program ignoring the durations that are written in brackets.

\begin{tabular}{|c|c|c|c|c|c|c|c|c|c|}
\hline 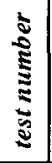 & 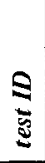 & 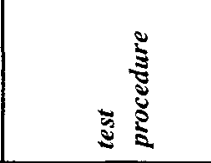 & $\stackrel{\bullet}{\Xi}$ & 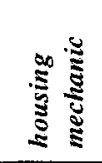 & 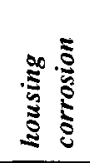 & 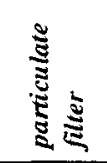 & 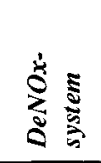 & 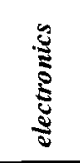 & \\
\hline & & & & $\beta=1.5$ & $\beta=2$ & $\beta=1.8$ & $\beta=1.3$ & $\beta=I$ & \\
\hline & & & & \multicolumn{5}{|c|}{ effective mileage (per component) } & comment/ test result \\
\hline 1 & $A$ & hydropuls test & kmiles & $1750]$ & 0 & 0 & 0 & 0 & $O K$ \\
\hline 2 & $H$ & spray test & kmiles & {$[750]$} & 2250 & {$[750]$} & 750 & 750 & crack; redesign of housing required \\
\hline 3 & $A$ & hydropuls test & kmiles & 750 & 0 & 0 & 0 & 0 & $O K$ \\
\hline 4 & $A$ & hydropuls test & kmiles & 750 & 0 & 0 & 0 & 0 & $O K$ \\
\hline 5 & $H$ & spray test & kmiles & 750 & 225 & {$[750]$} & 750 & 750 & $O K$ \\
\hline 6 & $F$ & customer testing & kmiles & 200 & 200 & {$[200]$} & 200 & 200 & filter cracked, redesign \\
\hline 7 & $G$ & alternating load & kmiles & 0 & 0 & 600 & {$[600]$} & 600 & DeNOx syst. failed due to prototyp built \\
\hline 8 & $H$ & spray test & kmiles & 750 & 225 & 750 & 750 & 750 & $O K$ \\
\hline 9 & $I$ & WHTC cycle & kmiles & 0 & 150 & [450] & 450 & 300 & filter damaged, human error operator \\
\hline 10 & $B$ & corrosion test & kmiles & 0 & 750 & 0 & 0 & 0 & $O K$ \\
\hline 11 & $D$ & city cycle & kniles & 100 & 300 & 300 & 300 & 200 & $O K$ \\
\hline 12 & $E$ & rough road test & kmiles & [700] & 21 & 21 & 21 & $2 I$ & housing cracked due to bad proto weld \\
\hline 13 & $D$ & city cycle & kmiles & 100 & 300 & 300 & 300 & 200 & $O K$ \\
\hline 14 & $C$ & express-run & kniles & 200 & 200 & 300 & 300 & 200 & $O K$ \\
\hline 15 & $l$ & WHTC cycle & kmiles & 0 & 150 & 450 & 450 & 300 & $O K$ \\
\hline 16 & $C$ & express-run & kmiles & 200 & 200 & 300 & 300 & 200 & $O K$ \\
\hline 17 & $F$ & customer testing & kmiles & 200 & 200 & 200 & 200 & 200 & $O K$ \\
\hline 18 & $F$ & customer testing & kmiles & 200 & 200 & 200 & 200 & 200 & $O K$ \\
\hline 19 & $F$ & customer testing & kniles & 200 & 200 & 200 & 200 & 200 & $O K$ \\
\hline & & $T T i$ & kmiles & 4400 & 5571 & 3621 & 5171 & 5071 & remark: only tests w/o issues are counted \\
\hline
\end{tabular}

Table 3.3 RDT documentation

Based on this information one has to make a statement on the demonstrated level of reliability. 
In Table 3.4, all durations in brackets [] were set to 0. Next, $T W_{i}$ is calculated for each component. This is substituted into equation (3.9) to calculate the LCL of component reliability. In this example a confidence level of $b=10 \%$ is applied.

\begin{tabular}{|c|c|c|c|c|c|c|c|c|}
\hline 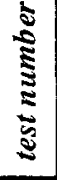 & $\begin{array}{l}\Xi \\
\vdots \\
\vdots \\
⿱ 亠 乂\end{array}$ & 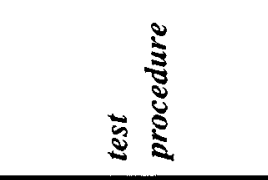 & $\stackrel{\Xi}{\Xi}$ & 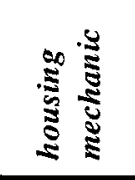 & 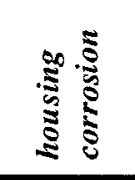 & 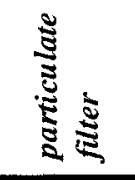 & 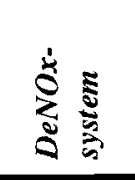 & \begin{tabular}{l}
3 \\
\multirow{3}{*}{} \\
$\frac{3}{0}$ \\
0
\end{tabular} \\
\hline & & & & $\beta=1.5$ & $\beta=2$ & $\beta=1.8$ & $\beta=1.3$ & $\beta=1$ \\
\hline & & & & \multicolumn{5}{|c|}{ effective mileage (per component) } \\
\hline$I$ & $A$ & hydropuls test & kmiles & 0 & 0 & 0 & 0 & 0 \\
\hline 2 & $H$ & spray test & kmiles & 0 & 2250 & 0 & 750 & 750 \\
\hline 3 & $A$ & hydropuls test & kmiles & 750 & 0 & 0 & 0 & 0 \\
\hline 4 & $A$ & hydropuls test & kmiles & 750 & 0 & 0 & 0 & 0 \\
\hline 5 & $H$ & spray test & kmiles & 750 & 225 & 0 & 750 & 750 \\
\hline 6 & $F$ & customer testing & kmiles & 200 & 200 & 0 & 200 & 200 \\
\hline 7 & $G$ & alternating load & kmiles & 0 & 0 & 600 & 0 & 600 \\
\hline 8 & $H$ & spray test & kmiles & 750 & 225 & 750 & 750 & 750 \\
\hline 9 & $I$ & WHTC cycle & kmiles & 0 & 150 & 0 & 450 & 300 \\
\hline 10 & $B$ & corrosion test & kmiles & 0 & 750 & 0 & 0 & 0 \\
\hline 11 & $D$ & city cycle & kmiles & 100 & 300 & 300 & 300 & 200 \\
\hline 12 & $E$ & rough road test & kmiles & 0 & 21 & 21 & 21 & 21 \\
\hline 13 & $D$ & city cycle & kmiles & 100 & 300 & 300 & 300 & 200 \\
\hline 14 & $C$ & express-run & kmiles & 200 & 200 & 300 & 300 & 200 \\
\hline 15 & $I$ & WHTC cycle & kmiles & 0 & 150 & 450 & 450 & 300 \\
\hline 16 & $C$ & express-run & kmiles & 200 & 200 & 300 & 300 & 200 \\
\hline 17 & $F$ & customer testing & kmiles & 200 & 200 & 200 & 200 & 200 \\
\hline 18 & $F$ & customer testing & kmiles & 200 & 200 & 200 & 200 & 200 \\
\hline 19 & $F$ & customer testing & kmiles & 200 & 200 & 200 & 200 & 200 \\
\hline & & $T W_{-} i$ & kmiles & 2171 & 2488 & 1410 & 2965 & 5071 \\
\hline & & Confidence Level & $b=0.1$ & & & & & \\
\hline & & $R \_L C L \_C(t 0=0)$ & $\%$ & $100.00 \%$ & $100.00 \%$ & $100.00 \%$ & $100.00 \%$ & $100.00 \%$ \\
\hline & & $R \_L C L_{-} C(t 0=50)$ & $\%$ & $99.20 \%$ & $99.91 \%$ & $99.44 \%$ & $98.87 \%$ & $97.76 \%$ \\
\hline & & $R \_L C L \_C(t O=100)$ & $\%$ & $97.75 \%$ & $99.63 \%$ & $98.05 \%$ & $97.23 \%$ & $95.56 \%$ \\
\hline & & $R \_L C L \_C(t 0=150)$ & $\%$ & $95.90 \%$ & $99.17 \%$ & $96.00 \%$ & $95.35 \%$ & $93.42 \%$ \\
\hline & & $R \_L C L \_C(t 0=200)$ & $\%$ & $93.76 \%$ & $98.52 \%$ & $93.38 \%$ & $93.32 \%$ & $91.32 \%$ \\
\hline & & $R \_L C L \_C(t 0=250)$ & $\%$ & $91.39 \%$ & $97.70 \%$ & $90.28 \%$ & $91.17 \%$ & $89.27 \%$ \\
\hline & & $R \_L C L \_C(t O=300)$ & $\%$ & $88.84 \%$ & $96.71 \%$ & $86.76 \%$ & $88.94 \%$ & $87.26 \%$ \\
\hline & & $R \_L C L \_C(t 0=350)$ & $\%$ & $86.15 \%$ & $95.55 \%$ & $82.91 \%$ & $86.66 \%$ & $85.31 \%$ \\
\hline & & $R \_L C L \_C(t O=400)$ & $\%$ & $83.35 \%$ & $94.22 \%$ & $78.79 \%$ & $84.34 \%$ & $83.39 \%$ \\
\hline
\end{tabular}

Table 3.4 Calculation of LCL of system reliability $R_{L C L, b, i}$ 
For example at duration $t_{0}=350$ kmiles the LCL of the reliability of component 1 to 5 is: $86.15 \% ; 95.55 \% ; 82.91 \% ; 86.66 \% ; 85.31 \%$

Multiplying these LCL of reliability would lead to a very low system reliability of only $50.45 \%$ :

$R_{\text {mult } ; L C L ; 10 \%}\left(t_{0}=350\right)=0.8615 * 0.9555 * 0.8291 * 0.8666 * 0.8531=0.5045$

According to the theorem (3.13) the LCL of system reliability is equal to the lowest of the system reliabilities. This leads to a much higher value of $82.91 \%$ :

$R_{S, L C L, b}\left(t_{0}=350\right)=\operatorname{Min}(0.8615 ; 0.9555 ; 0.8291 ; 0.8666 ; 0.8531)=0.8291$

It is obvious that the limiting component for LCL of system reliability can change depending on the considered time $t_{0}$. In this example up to $t_{0}=250$ component 5 is limiting. From $t_{0}=300$ and above component 3 determines the LCL of system reliability.

The resulting function for LCL of system reliability in respect to point $t$ is typically not a continuous function. So the LCL of system reliability does not follow the Weibull law although the individual components do. 
The following chart illustrates the LCL of system and component reliability depending on the duration $t_{0}$ :

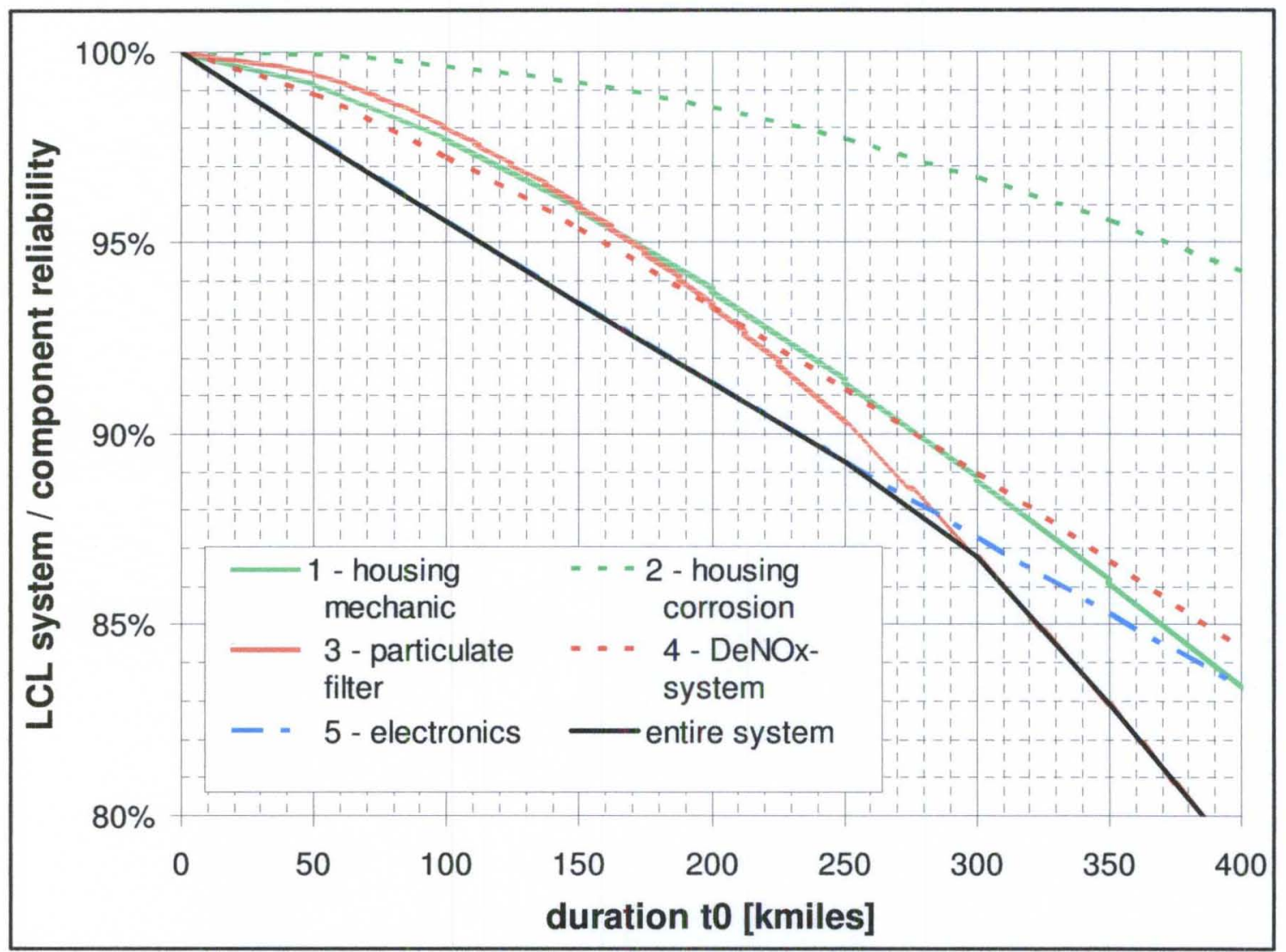

Figure 3.1 LCL of system and component reliability

Table 3.5 shows the LCL of system $B_{X}$-lifetime for different values of $X$, the maximum share of failed systems permitted, calculated according to equation (3.17). Similar to the calculation of the LCL of system reliability the limiting component can change depending on the value for $X$. In this example, component 5 is limiting up to $X=10 \%$. For $X=20 \%$ and above, component 3 determines the LCL of system $B_{X}$-lifetime. 


\begin{tabular}{|c|c|c|c|c|c|c|c|c|}
\hline & & $\stackrel{\Xi}{\Xi}$ & 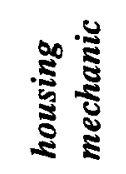 & 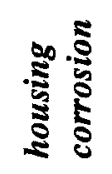 & 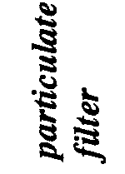 & 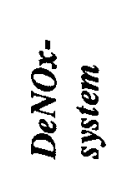 & 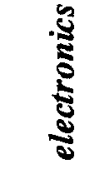 & เู \\
\hline & & & $\beta=1.5$ & $\beta=2$ & $\beta=1.8$ & $\beta=1.3$ & $\beta=1$ & \\
\hline$T W_{-} i$ & & kmiles & 2171 & 2488 & 1410 & 2965 & 5071 & \\
\hline B1-lifetime & $1 \%$ & kmiles & 58.0 & 164.4 & 68.9 & 45.4 & 22.1 & 22.1 \\
\hline B2 - Lifetime & $2 \%$ & kmiles & 92.3 & 233.1 & 101.5 & 77.6 & 44.5 & 44.5 \\
\hline B5 - Lifetime & $5 \%$ & kmiles & 171.8 & 371.4 & 170.4 & 158.9 & 113.0 & 113.0 \\
\hline B10-Lifetime & $10 \%$ & kmiles & 277.7 & 532.3 & 254.2 & 276.5 & 232.0 & 232.0 \\
\hline B20 - Lifetime & $20 \%$ & kmiles & 458.0 & 774.6 & 385.6 & 492.4 & 491.4 & 385.6 \\
\hline B30 - Lifetime & $30 \%$ & kmiles & 626.1 & 979.3 & 500.4 & 706.4 & 785.5 & 500.4 \\
\hline B50 - Lifetime & $50 \%$ & kmiles & 975.0 & 1365.2 & 723.8 & 1177.6 & 1526.5 & 723.8 \\
\hline
\end{tabular}

Table 3.5 Calculation of $b=10 \%$ LCL of system $B_{X}-$ lifetime

\subsection{Advantages of the Proposed Method to Calculate LCL of System Reliability}

If the validation of the theorems according to equations (3.15) and (3.17) is successful, then the following improvements for reliability considerations of systems after RDT with zero failures will be achieved:

- Making statements for LCL reliability for a system for different Weibull factors $\beta_{i}$ and component specific testing durations will be possible.

- Making statements for LCL $B_{X}$ - lifetime for a system for different Weibull factors $\beta_{i}$ and component specific testing durations will be possible.

- LCL of system reliability $R_{S, L C L, b}$ will be much higher than multiplying the LCL of each component according to the single component approach according to equation (3.6).

- The LCL of reliability and $B_{X}$ - lifetime can be integrated into a framework for further optimization of the test programs for RDT. 


\section{PROOF OF THE THEOREMS}

\subsection{Proof of the First Theorem (System Reliability) for $n=2$}

The first theorem according to equation (3.15) defines the $b$ LCL of system reliability $R_{S ; L C L ; b}$ at time $t_{0}$ of a system consisting of $n$ Weibull components (with shape parameter $\left.\beta_{i}\right)$. In this section we first consider a system with only two components $(n=2)$. The components of the system are exposed to the Weibull equivalent single test duration $T W_{i}$ with zero failures occurring during RDT as defined in Chapter 3.2. According to the theorem of equation (3.15) the LCL of system reliability is then:

$$
R_{S ; L C L ; b}\left(t_{0}\right)=\operatorname{Min}_{i}\left[e^{\left(\frac{t_{0}^{\beta_{i}} \ln (b)}{T W_{i}^{\beta_{i}}}\right)}\right]=\operatorname{Min}_{i}\left[b^{\left(\frac{t_{0}^{\beta_{i}}}{T W_{i}^{\beta_{i}}}\right)}\right]
$$

Figure 4.1 indicates the probability of zero failures in RDT depending on the actual system reliability. The higher the system reliability is, the higher is also the probability of zero failures during a certain RDT program.

According to Chapter 2.4 , the type II error is related to a test of the following hypothesis on the real value for reliability $R_{a, S}$ of the system:

- null hypothesis

$$
\begin{aligned}
& H_{0}: R_{a, S}\left(t_{0}\right)=R_{S ; L C L ; b}\left(t_{0}\right) \\
& H_{l}: R_{a, S}\left(t_{0}\right)<R_{S ; L C L ; b}\left(t_{0}\right)
\end{aligned}
$$

- alternative hypothesis

The null hypothesis will not be rejected if zero failures occur in RDT. This is a false decision when the real value $R_{a, S}$ is lower than $R_{S ; L C L ; b}\left(t_{0}\right)$. This is a type II error and should have a probability less or equal than the confidence level $b$. 
On the other hand, the null hypothesis $H_{0}$ will be rejected if one or more failures occur in RDT. This is erroneous decision if the actual reliability of the system $R_{a ; s}$ is equal or higher than what is stated in $H_{0}$. This is called a type I error.

The LCL on system reliability is related to a type II error. In equation (3.15), or (4.1) respectively, the variable $b$ denotes the risk of a type II error.

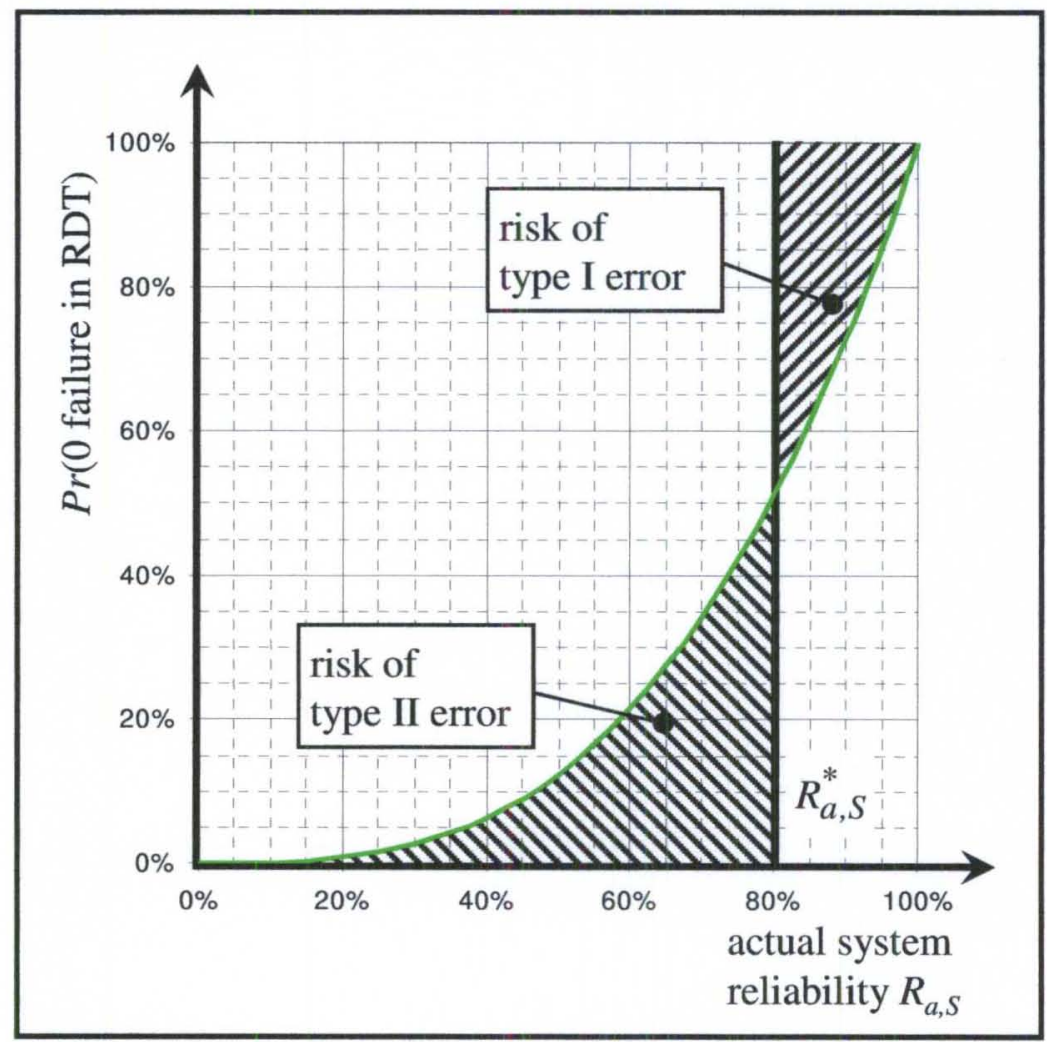

Figure 4.1 Probability of zero failure in RDT at different system reliability levels

Figure 4.2 illustrates the meaning of LCL: the probability of zero failures is less or equal to $b$ for the case that the actual system reliability is $R_{S ; L C L ; b}$. 


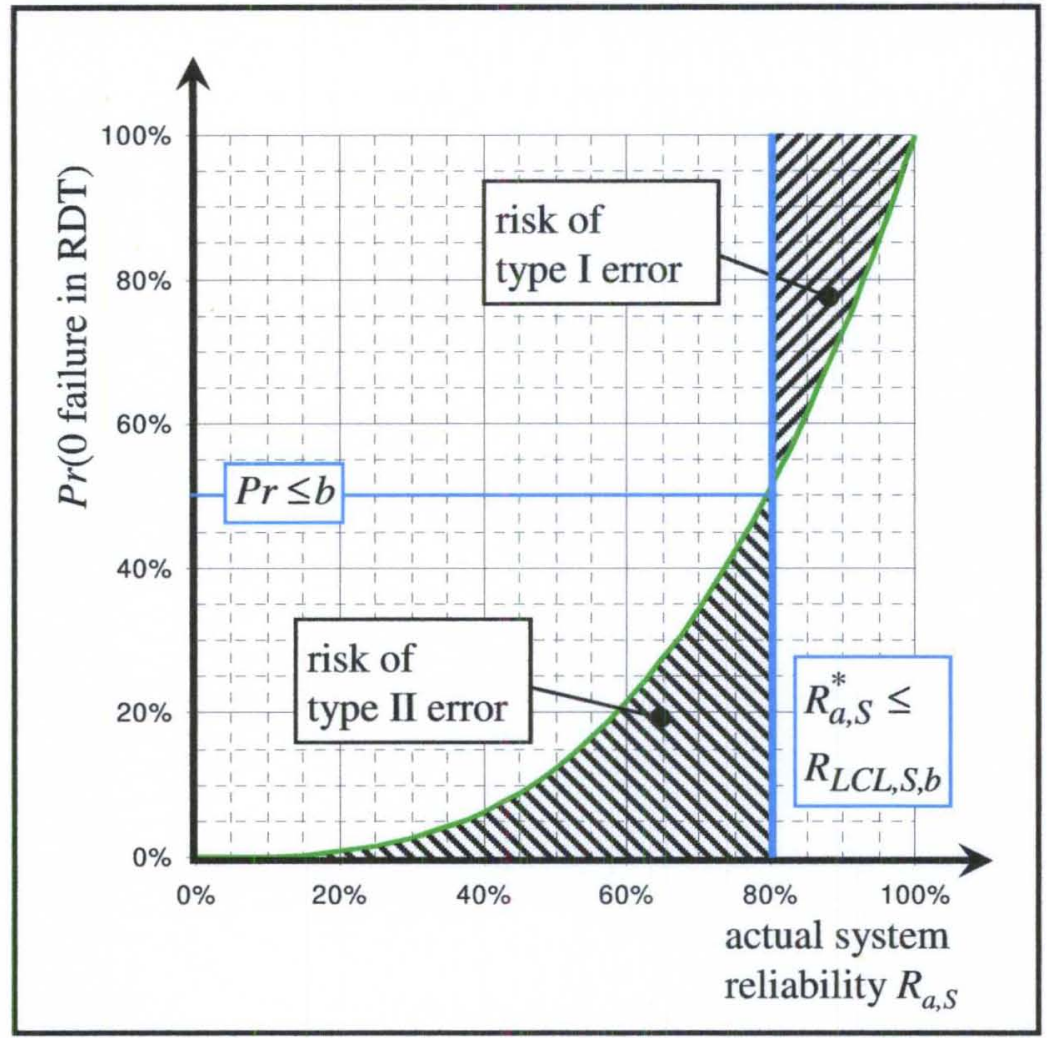

Figure 4.2 Illustration of the theorem for LCL of system reliability

Let $R_{a ; i}$ denote the actual reliability of the component $i$ and $\theta_{a ; i}$ the respective Weibull scale parameter. Each component follows the Weibull distribution according to equation (2.11). So the actual reliability of component $i$ at duration $t_{0}$ is given as:

$R_{a, i}\left(t_{0}\right)=e^{-\left(\frac{t_{0}}{\theta_{a, i}}\right)^{\beta_{i}}}$

The actual reliability $R_{a ; S}$ for the serial system with two components at duration $t_{0}$ is then:

$R_{a, S}\left(t_{0}\right)=\prod_{i=1}^{2} e^{-\left(\frac{t_{0}}{\theta_{a, i}}\right)^{\beta_{i}}}=e^{-\left[\left(\frac{t_{0}}{\theta_{a, 1}}\right)^{\beta_{1}}+\left(\frac{t_{0}}{\theta_{a, 2}}\right)^{\beta_{2}}\right]}$ 
The probability of zero failures occurring on component $i$ during the entire RDT program with $j=1 . . m$ tests is:

$\operatorname{Pr}($ Ofailures; comp $i)=\prod_{j=1}^{m} e^{-\left(\frac{t_{i j}}{\theta_{a, i}}\right)^{\beta_{i}}}=e^{-\sum_{j=l}^{m}\left(\frac{t_{i j}}{\theta_{a, i}}\right)^{\beta_{i}}}=e^{-\left(\frac{T W_{i}}{\theta_{a, i}}\right)^{\beta_{i}}}$

The probability of zero failures occurring on the two components of the system is then:

$\operatorname{Pr}($ Ofailures, Sys $)=\prod_{i=1}^{2} \prod_{j=1}^{m} e^{-\left(\frac{t_{i j}}{\theta_{a, i}}\right)^{\beta_{i}}}=\prod_{i=1}^{2} e^{-\sum_{j=1}^{m}\left(\frac{t_{i j}}{\theta_{a, i}}\right)^{\beta_{i}}}$

$\operatorname{Pr}($ Ofailures, Sys $)=e^{-\left[\left(\frac{T W_{1}}{\theta_{a, 1}}\right)^{\beta_{1}}+\left(\frac{T W_{2}}{\theta_{a, 2}}\right)^{\beta_{2}}\right]}$

To prove the theorem (4.1), that the following statement has to be verfied:

The probability of zero failures occurring in RDT is less or equal than $b$ for the case that the actual value of the system reliability $R_{a ; S}\left(t_{0}\right)$ is less or equal to $R_{S ; L C L ; b}\left(t_{0}\right)$.

Since the probability of zero failures in RDT increases with the actual system reliability, it is sufficient to verify this for the case of $R_{a ; S}\left(t_{0}\right)$ being equal to $R_{S ; L C L ; b}\left(t_{0}\right)$.

For the first part the probability of zero failures occurring is less or equal than $b$ is defined by the following equation:

$\operatorname{Pr}($ O failures, Sys $)=\prod_{i=1}^{2} e^{-\left(\frac{T W_{i}}{\theta_{a, i}}\right)^{\beta_{i}}}=e^{-\left[\left(\frac{T W_{1}}{\theta_{a, 1}}\right)^{\beta_{1}}+\left(\frac{T W_{2}}{\theta_{a, 2}}\right)^{\beta_{2}}\right]} \leq b$ 
The second part of statement above sets the actual system reliability $R_{a ; s}\left(t_{0}\right)$ equal to $R_{S ; L C L ; b}\left(t_{0}\right) .:$

$$
R_{a ; S}\left(t_{0}\right)=e^{-\left[\left(\frac{t_{0}}{\theta_{a, 1}}\right)^{\beta_{1}}+\left(\frac{t_{0}}{\theta_{a, 2}}\right)^{\beta_{2}}\right]}=R_{S ; L C L ; b}\left(t_{0}\right)
$$

According to the theorem and (4.1) $R_{S ; L C L ; b}\left(t_{0}\right)$ is:

$$
R_{S ; L C L ; b}\left(t_{0}\right)=\operatorname{Min}_{i}\left[e^{\left(\frac{t_{0}^{\beta_{i} * \ln (b)}}{T W_{i}^{\beta_{i}}}\right)}\right]=\operatorname{Min}_{i}\left[b^{\left(\frac{t_{0}^{\beta_{i}}}{T W_{i}^{\beta_{i}}}\right)}\right]
$$

Let component $i=1$ be the limiting component that determines the value of the LCL of system reliability according to equation (4.8). Considering $0<b<1$ this leads to:

$\frac{t_{0}^{\beta_{1}}}{T W_{1}^{\beta_{1}}} \geq \frac{t_{0}^{\beta_{2}}}{T W_{2}^{\beta_{2}}}$

The LCL of system reliability (4.8) with $i=l$ as the limiting component is then:

$$
R_{S ; L C L ; b}\left(t_{0}\right)=\operatorname{Min}_{i}\left[b^{\left(\frac{t_{0}^{\beta_{i}}}{T W_{i}^{\beta_{i}}}\right)}\right]=b^{\left(\frac{t_{0}^{\beta_{I}}}{T W_{I}^{\beta_{I}}}\right)}
$$

Substituting (4.10) into (4.7):

$$
e^{-\left[\left(\frac{t_{0}}{\theta_{a, I}}\right)^{\beta_{1}}+\left(\frac{t_{0}}{\theta_{a, 2}}\right)^{\beta_{2}}\right]}=b^{\left(\frac{t_{0}^{\beta_{I}}}{T W_{1}^{\beta_{I}}}\right)}
$$


Solving (4.11) for $b$ :

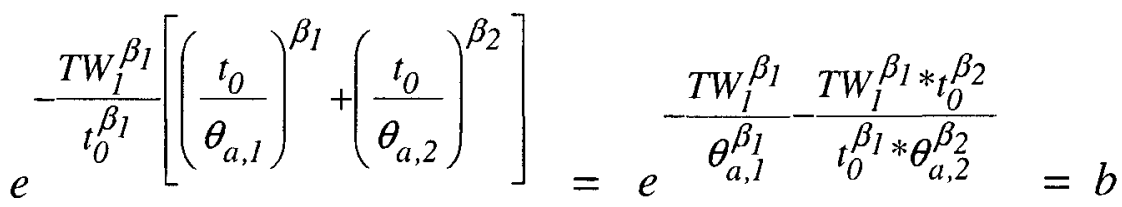

Substituting (4.12) into inequality (4.6):

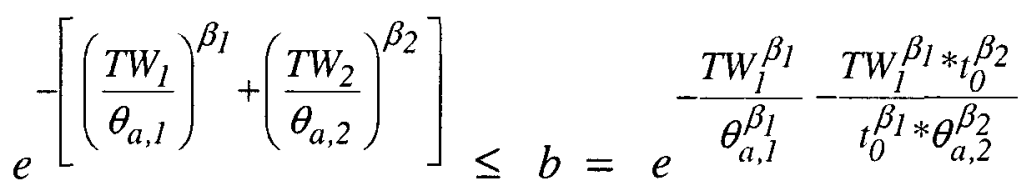

Inequality (4.13) can be simplified by taking the log on both sides:

$$
\begin{aligned}
& -\left(\frac{T W_{1}}{\theta_{a, 1}}\right)^{\beta_{1}}-\left(\frac{T W_{2}}{\theta_{a, 2}}\right)^{\beta_{2}} \leq-\frac{T W_{l}^{\beta_{1}}}{\theta_{a, 1}^{\beta_{1}}}-\frac{T W_{l}^{\beta_{1} * t_{0}^{\beta_{2}}}}{t_{0}^{\beta_{l} * \theta_{a, 2}^{\beta_{2}}}} \\
& -\frac{T W_{2}^{\beta_{2}}}{\theta_{a, 2}^{\beta_{2}}} \leq-\frac{T W_{1}^{\beta_{1} *} t_{0}^{\beta_{2}}}{t_{0}^{\beta_{1}} \theta_{a, 2}^{\beta_{2}}} \\
& -\left(\frac{T W_{2}}{t_{0}}\right)^{\beta_{2}} \leq-\left(\frac{T W_{1}}{t_{0}}\right)^{\beta_{l}}
\end{aligned}
$$

The inequality (4.14) is equivalent to inequality (4.9) and is therefore fulfilled as long as component $i=1$ is the limiting component for the determination of the LCL of system reliability. Of course this proof would also be valid if component $i=2$ was the limiting component for the LCL system of system reliability. In this case all indices in (4.9) to (4.14) would be switched from 1 to 2 and vice versa.

\section{Consequence:}

Since the inequality (4.14) is a correct statement the theorem is mathematically proven for two components $(n=2)$. 


\subsection{General Proof of the First Theorem (System Reliability) ( $n$ Components)}

In the following the proof of the first theorem is extended from two components to the general case of $n$ components.

The first theorem according to equation (3.15) defines the $b$ LCL of system reliability $R_{S ; L C L ; b}$ at time $t_{0}$ of a system consisting of $n$ Weibull components (with shape parameter $\beta_{i}$ ). The components of systems are exposed to the total Weibull equivalent single test duration $T W_{i}$ with zero failures occurring during RDT. So the following equation has to be proven for the general case with $n$ components:

$R_{S ; L C L ; b}\left(t_{0}\right)=\operatorname{Min}_{i}\left[e^{\left(\frac{t_{0}^{\beta_{i} * \ln (b)}}{T W_{i}^{\beta_{i}}}\right)}\right]=\operatorname{Min}_{i}\left[b^{\left(\frac{t_{0}^{\beta_{i}}}{T W_{i}^{\beta_{i}}}\right)}\right]$

The areas for type I and type II error were illustrated in Figure 4.1 and Figure 4.2 in the previous section. Let $R_{a ; i}$ denote the actual reliability of component $i$ and $\theta_{a ; i}$ the respective scale parameter. Each component is assumed to have a Weibull life distribution. So the respective reliability at duration $t_{0}$ yields to:

$R_{a, i}\left(t_{0}\right)=e^{-\left(\frac{t_{0}}{\theta_{a, i}}\right)^{\beta_{i}}}$

The reliability of the serial system of Weibull components is illustrated in Figure 4.3. Its actual reliability $R_{a ; S}$ at duration $t_{0}$ is then:

$R_{a, S}\left(t_{0}\right)=\prod_{i=1}^{n} e^{-\left(\frac{t_{0}}{\theta_{a, i}}\right)^{\beta_{i}}}=e^{-\sum_{i=1}^{n}\left(\frac{t_{0}}{\theta_{a, i}}\right)^{\beta_{i}}}$ 


\section{Serial System with n Weibull Components}

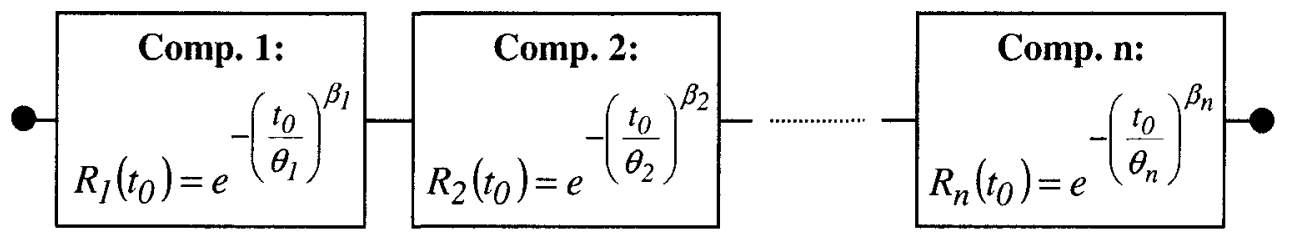

$$
R_{S}\left(t_{0}\right)=\prod_{i=1}^{n} e^{-\left(\frac{t_{0}}{\theta_{i}}\right)^{\beta_{i}}}=e^{-\sum_{i=1}^{n}\left(\frac{t_{0}}{\theta_{i}}\right)^{\beta_{i}}}
$$

Figure 4.3 Reliability of a serial system with Weibull components

The probability of zero failure occurring on component $i$ during the entire RDT program with $j=1 . . m$ tests is:

$\operatorname{Pr}($ O failures; comp $i)=\prod_{j=1}^{m} e^{-\left(\frac{t_{i j}}{\theta_{a, i}}\right)^{\beta_{i}}}=e^{-\sum_{j=1}^{m}\left(\frac{t_{i j}}{\theta_{a, i}}\right)^{\beta_{i}}}=e^{-\left(\frac{T W_{i}}{\theta_{a, i}}\right)^{\beta_{i}}}$

The probability of zero failure occurring on all components $i=1 . . n$ is then:

$\operatorname{Pr}($ O failures, Sys $)=\prod_{i=l}^{n} \prod_{j=l}^{m} e^{-\left(\frac{t_{i j}}{\theta_{a, i}}\right)^{\beta_{i}}}=\prod_{i=l}^{n} e^{-\sum_{j=l}^{m}\left(\frac{t_{i j}}{\theta_{a, i}}\right)^{\beta_{i}}}=\prod_{i=l}^{n} e^{-\left(\frac{T W_{i}}{\theta_{a, i}}\right)^{\beta_{i}}}$ 
To prove the theorem (4.15), the following statement has to be verified:

The probability of zero failures occurring in RDT is less or equal than $b$ for the case that the actual value of the system reliability $R_{a ; S}\left(t_{0}\right)$ is less or equal to $R_{S ; L C L ; b}\left(t_{0}\right)$.

Since the probability of zero failures in RDT increases with the actual system reliability, it is sufficient to verify this for the case of $R_{a ; S}\left(t_{0}\right)$ being equal to $R_{S ; L C L ; b}\left(t_{0}\right)$.

For the first part the probability of zero failures occurring is less or equal than $b$ is defined by the following equation:

$\operatorname{Pr}($ Ofailures, Sys $)=\prod_{i=1}^{n} e^{-\left(\frac{T W_{i}}{\theta_{a, i}}\right)^{\beta_{i}}}=e^{-\sum_{i=1}^{n}\left(\frac{T W_{i}}{\theta_{a, i}}\right)^{\beta_{i}} !} \leq b$

The second part of statement above sets the actual system reliability $R_{a ; S}\left(t_{0}\right)$ equal to $R_{S ; L C L ; b}\left(t_{0}\right)$ :

$$
R_{a, S}\left(t_{0}\right)=e^{-\sum_{i=1}^{n}\left(\frac{t_{0}}{\theta_{a, i}}\right)^{\beta_{i}} !}=R_{S ; L C L ; b}\left(t_{0}\right)
$$

According to (4.15) $R_{S ; L C L ; b}\left(t_{0}\right)$ is:

$$
R_{S ; L C L ; b}\left(t_{0}\right)=\operatorname{Min}_{i}\left[b^{\left(\frac{t_{0}^{\beta_{i}}}{T W_{i}^{\beta_{i}}}\right)}\right]
$$


Substituting (4.22) into (4.21):

$$
e^{-\sum_{i=1}^{n}\left(\frac{t_{0}}{\theta_{a, i}}\right)^{\beta_{i}}}=\operatorname{Min}_{i}\left[b^{\left(\frac{t_{0}^{\beta_{i}}}{T W_{i}^{\beta_{i}}}\right)}\right]
$$

Let $Y_{i}$ be: $Y_{i}=\left(\frac{T W_{i}^{\beta_{i}}}{t_{0}^{\beta_{i}}}\right)=\left(\frac{T W_{i}}{t_{0}}\right)^{\beta_{i}}$ with $Y_{i}>0$.

High values of $Y_{i}$ mean that the Weibull equivalent single test duration $T W_{i}$, which is reflecting the entire testing of a certain component $i$, is large in comparison to the time $t_{0}$. Substituting this into (4.23) yields to:

$$
e^{-\sum_{i=1}^{n}\left(\frac{t_{0}}{\theta_{a, i}}\right)^{\beta_{i}}}=\operatorname{Min}_{i}\left[b^{\left(\frac{t_{0}^{\beta_{i}}}{T W_{i}^{\beta_{i}}}\right)}\right]=\operatorname{Min}_{i}\left[b^{\left(l / Y_{i}\right)}\right]
$$

The confidence level $b$ is defined for $0<b<1$. So the term $b^{\left(1 / Y_{i}\right)}$ is smaller if the value of $Y_{i}$ gets smaller. With $Y_{\min }=\operatorname{Min}_{i}\left[Y_{i}\right]$ the equation (4.24) can be transformed into:

$$
e^{-\sum_{i=1}^{n}\left(\frac{t_{0}}{\theta_{a, i}}\right)^{\beta_{i}}}=\operatorname{Min}_{i}\left[b^{\left(1 / Y_{i}\right)}\right]=b^{\left(1 / Y_{\min }\right)}
$$


Solving (4.25) for $b$ results in:

$$
\left[e^{\left.-\sum_{i=1}^{n}\left(\frac{t_{0}}{\theta_{a, i}}\right)^{\beta_{i}}\right]^{Y_{\min }}}=e^{-\sum_{i=1}^{n}\left[Y_{\min } *\left(\frac{t_{0}}{\theta_{a, i}}\right)^{\beta_{i}}\right]}=b\right.
$$

Substituting (4.26) into (4.20) gives:

$$
e^{-\sum_{i=l}^{n}\left(\frac{T W_{i}}{\theta_{a, i}}\right)^{\beta_{i}}} \leq b=e^{-\sum_{i=l}^{n}\left[Y_{\min } *\left(\frac{t_{0}}{\theta_{a, i}}\right)^{\beta_{i}}\right]}
$$

Substituting $T W_{i}^{\beta_{i}}=t_{0}^{\beta_{i}} * Y_{i}$ into (4.27) yields to:

$$
e^{-\sum_{i=l}^{n}\left[Y_{i} *\left(\frac{t_{0}}{\theta_{a, i}}\right)^{\beta_{i}}\right]} \leq e^{-\sum_{i=l}^{n}\left[Y_{\min } *\left(\frac{t_{0}}{\theta_{a, i}}\right)^{\beta_{i}}\right]}
$$

Taking the log and multiplying with (-1) on both sides:

$$
\sum_{i=1}^{n}\left[Y_{i} *\left(\frac{t_{0}}{\theta_{a, i}}\right)^{\beta_{i}}\right] \geq \sum_{i=1}^{n}\left[Y_{\min } *\left(\frac{t_{0}}{\theta_{a, i}}\right)^{\beta_{i}}\right] \text { with } t_{0}, \theta_{a, i}, \beta_{i}>0
$$

(4.29) will be fulfilled if the following statement is fulfilled for each component $i$ :

$$
Y_{i} *\left(\frac{t_{0}}{\theta_{a, i}}\right)^{\beta_{i}} \geq Y_{\min } *\left(\frac{t_{0}}{\theta_{a, i}}\right)^{\beta_{i}} \text { (for all components } i \text { ) }
$$


The following term is positive for all components $\mathrm{i}$ :

$$
\left(\frac{t_{0}}{\theta_{a, i}}\right)^{\beta_{i}} \geq 0 \text { with } t_{0}, \theta_{a, i}, \beta_{i}>0
$$

Therefore (4.30) can be further simplified to:

$$
Y_{i} \geq Y_{\min }(\text { for all components } i)
$$

The inequality (4.32) is per definition fulfilled for all components $i$. This means also that inequality (4.30) and subsequently also inequality (4.29) are fulfilled.

\section{Consequence:}

Since the inequality (4.32) and (4.29) are correct statements, the first theorem according to (3.15) is mathematically proven.

Figure 4.4 shows that $R_{S ; L C L ; b}$, the $b$ LCL of system reliability, is the minimum of the $b$ LCL of component reliability defined according to equation (3.10). It is obvious that the limiting component that determines the LCL of system reliability can change depending on the considered duration $t_{0}$ the reliability statement is related to. 


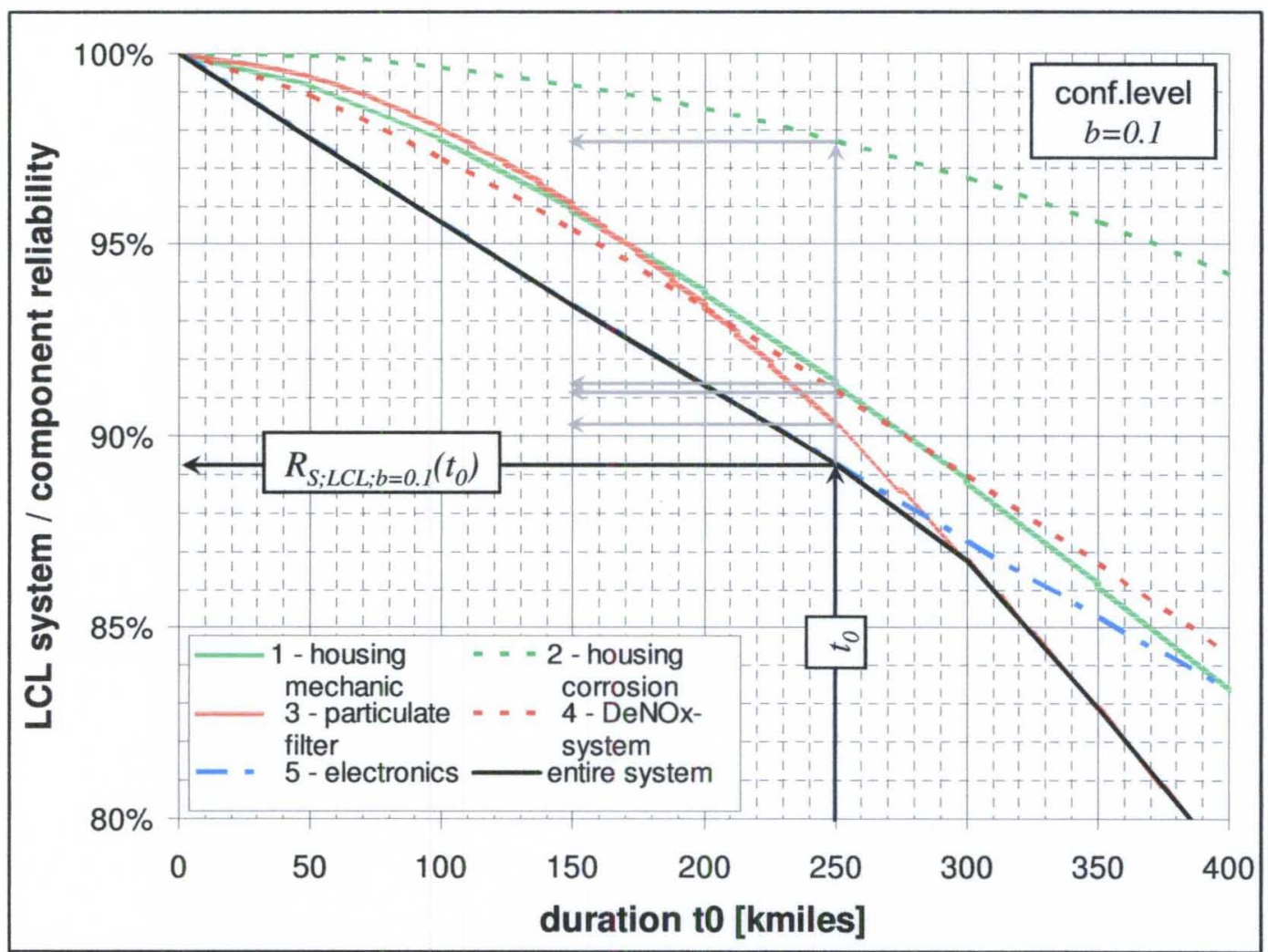

Figure 4.4 LCL for system reliability as the minimum of LCLs of component reliabilities

\subsection{Proof of the Second Theorem (System $B_{X}$ - lifetime)}

The second theorem, as formulated in equation (3.17), is related to the $b$ LCL of $B_{X}$ lifetime of a system consisting of $n$ Weibull components (with shape parameter $\beta_{i}$ ). The components of the system are exposed to the Weibull equivalent single test duration $T W_{i}$ with zero failures occurring during RDT. Equation (3.17) states:

$$
B_{X ; S ; L C L ; b}=\operatorname{Min}_{i}\left[\left(\frac{\ln \left(1-\frac{x}{100 \%}\right) * T W_{i}^{\beta_{i}}}{\ln (b)}\right)^{1 / \beta_{i}}\right]
$$


The $b$ LCL of $B_{X}$ - lifetime of a Weibull item that was tested with zero failures is ${ }^{17}$ :

$B_{X ; L C L ; b}=\frac{T W *\left[1-\frac{X}{100 \%}\right]^{1 / \beta}}{\ln (1 / b)^{1 / \beta}}$

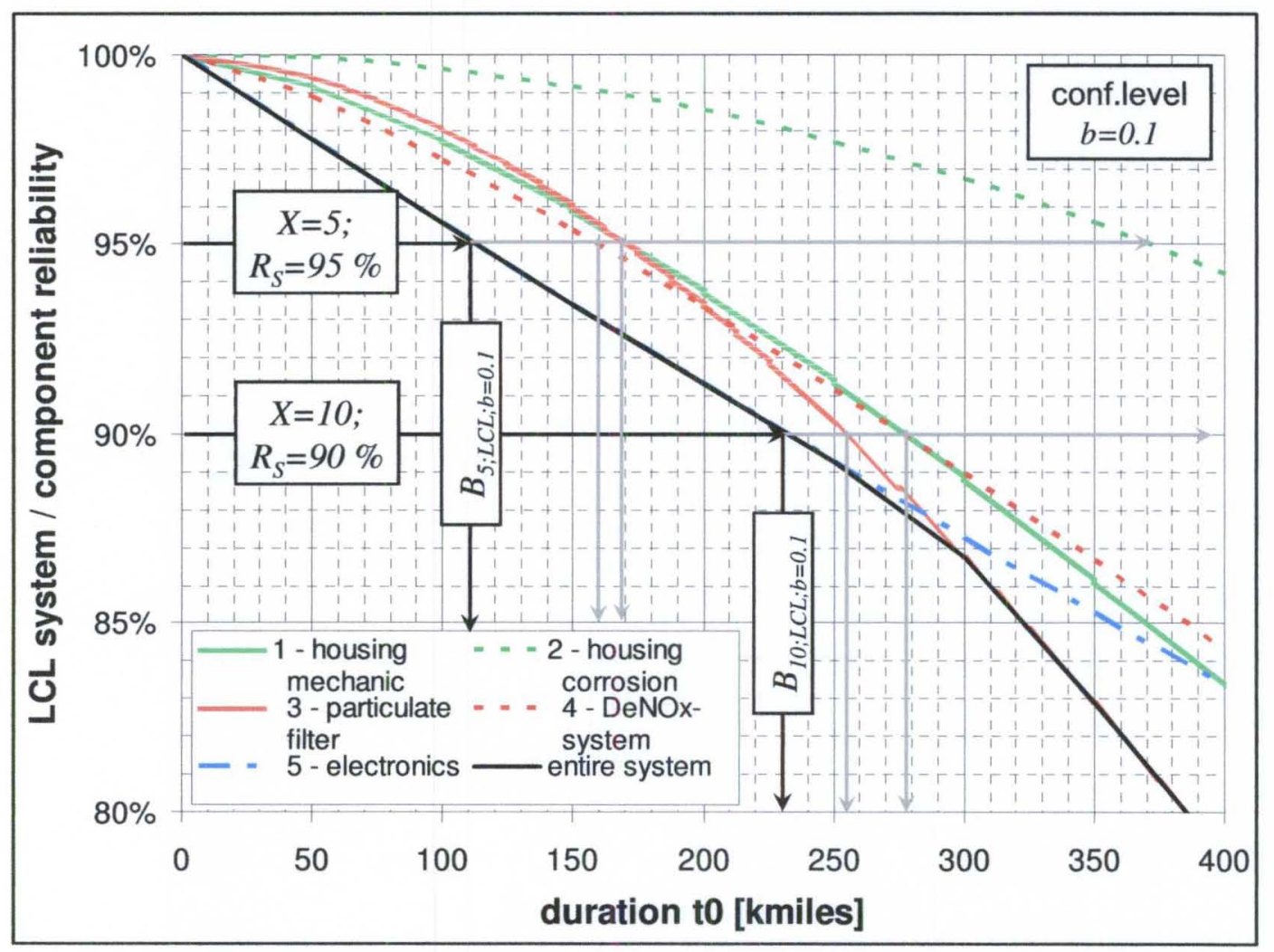

Figure 4.5 LCL of system $B_{10}$ as the minimum of the LCLs of component $B_{10}$

${ }^{17}$ see equation (App.4.10) in Appendix 4 
Figure 4.5 shows the same curves as Figure 4.4 above has showed. But differently to the case before, the diagram is now horizontally entered at a given reliability level which is exactly $(100 \%-X \%)$. In this example the reliability is 0.9 since $X=10$. There are different durations at which the LCL of component reliability is 0.9 . The lowest of these durations is the $b$ LCL of system $B_{X}$ - lifetime. This is exactly what is stated by the second theorem.

Since the first theorem was proven mathematically and this is the basis for both Figure 4.4 and Figure 4.5, there is no need to additionally prove the second theorem mathematically. The proof of the second theorem goes on with logical deduction based on the graphs in Figure 4.5. This is possible since the $B_{X}$ - lifetime, being the inversion of the CDF, is closely related to the reliability.

The reason for not needing a second mathematical proof is the relation between the reliability and $B_{X}$ - lifetime that goes along the cumulative distribution function CDF. The $B_{X}$ - lifetime is the inverse function of the CDF and the reliability is $l$ minus the CDF.

Comparable to the system reliability, it depends on the value of $X$ (the maximum share of systems that have failed), which one is the limiting component for the calculation of the LCL of $B_{X}$ - lifetime. 


\section{SENSITIVITY OF THE WEIBULL SHAPE PARAMETER ESTIMATION}

\subsection{Introduction to $\beta$ Sensitivity}

In the previous chapters the Weibull shape parameters $\beta_{i}$ for the different components were considered to be known for the case where the $b \mathrm{LCL}$ of system reliability $R_{S ; L C L ; b}$ is calculated (according to equation (3.15)). This could be, for example, based on past experience with similar components or the basic understanding of the system and the respective failure behavior.

In this chapter a model for the analysis of the influence of the component Weibull shape parameters $\beta_{i}$ on the $b$ LCL of system reliability $R_{S ; L C L ; b}$ will be developed. It allows us to quantify the sensitivity of the estimation of $\beta_{i}$.

We propose a non-linear program (NLP) with the objective LCL of system reliability $R_{S ; L C L ; b}$ to calculate the sensitivity of $\beta_{i}$. The decision variables of the NLP are the component Weibull parameters $\beta_{i}$. Each $\beta_{i}$ is between an upper and a lower bound, i.e. $\beta_{\min ; i} \leq \beta_{i} \leq \beta_{\max ; i}$. As described above for $\beta_{i}$, the upper and lower bounds of $\beta_{m i n ; i}$ and $\beta_{\text {max } ;}$, also have to be determined by past experience or system knowledge. But it is of course much easier to estimate an interval rather than a fixed value. This means that the associated risk resulting from an erroneous estimation is significantly lower and the respective statements are much more on the conservative side. So the loss in total system knowledge - meaning a range for $\beta_{i}$ instead of fixed values - results in a lower LCL of system reliability. 
The model applies to the case of finding the lowest value of the LCL of reliability $R_{S ; L C L ; b}$ is within the possible ranges of the $\beta_{i}$. The optimization model searches for the values of the component Weibull shape parameters that result in the lowest LCL of system reliability with the given test durations $t_{j}$ and acceleration factors $A F_{i j}$. It was mentioned in section 2.5 .2 of the literature review ${ }^{18}$ that there is there is a single value of the Weibull parameter $\beta$ that leads to the minimum LCL of reliability. Note that opposed to the model presented here, the cited papers in this area are related to single items only and not to systems.

Since the first theorem, (3.15), is based on similar approaches for zero failures as the mentioned research on single Weibull items and the LCL of system reliability is just the lowest of them, there is consequently a single value $\beta_{i *}$ of one specific component $i^{*}$ that determines the LCL of system reliability. This component $i *$ is then the limiting component for the LCL of system reliability and the respective LCL of component reliability is the overall lowest among all components. Depending on the upper and lower bounds of $\beta_{i}$ as well as on the duration $t_{0}$ the limiting component can change (but does not have to). If there is no limitation of the component Weibull parameters (i.e. $\beta_{i ; \min } \approx 0$; $\beta_{i ; m a x} \approx \infty$ ), the limiting component $i^{*}$ for the LCL of system reliability is the same for all values of the duration $t_{0}$.

\subsection{Optimization Model to Consider $\beta$ Sensitivity}

The respective NLP for the $b$ LCL of system reliability considers the uncertainty of $\beta_{i}$. It minimizes the $b$ LCL of system reliability, using the component Weibull parameters $\beta_{i}$ as the decision variables.

\footnotetext{
${ }^{18}$ see Huang and Porter (1991), Huang (1997) as well as Lu and Wang (2008)
} 
The parameters of the NLP (5.1) are:

- information about the conducted reliability tests:

test durations $t_{j}$, acceleration factors $A F_{i j}$, boolean variables to indicate the test relevance $o_{i j}$

- $\beta_{\min ; i}$ and $\beta_{\max ; i}$, the upper and lower bound for $\beta_{i}$ :

- duration $t_{0}$

- confidence level $b$

The resulting NLP is given in the equation set (5.1):

$$
\begin{aligned}
& \text { Minimize } R_{S ; L C L ; b} \\
& \text { subject to } \\
& \text { a) } R_{S ; L C L ; b}\left(t_{0}\right)=\operatorname{Min}_{i}\left[\begin{array}{c}
\left(\frac{t_{0}^{\beta_{i}}}{T W_{i}^{\beta_{i}}}\right) \\
\end{array}\right] \\
& \text { b) } T W_{i}=\sqrt[\beta]{\sum_{j=l}^{m}\left(t_{j} * A F_{i j} * o_{i j}\right)^{\beta_{i}}} \quad \text { for } i=1, \ldots, n \\
& \text { c) } \beta_{\min ; i} \leq \beta_{i} \leq \beta_{\max ; i} \quad \text { for } i=1, . ., n \\
& \text { d) } t_{0} ; A F_{i j} ; \beta_{i} \geq 0 \text { for } i=1, . ., n ; \text { for } j=1, . ., m \\
& \text { e) } 0 \leq b \leq 1 \\
& \text { f) } o_{i j}=[0 ; 1] \quad \text { for } i=1, . ., n ; \text { for } j=1, . ., m
\end{aligned}
$$


Where:

a) calculation of the objective, $R_{S ; L C L ; b}$, according to the first theorem (3.15)

b) calculation of the Weibull equivalent single test duration $T W_{i}$ according to (3.5) for all components $i$

c) $\beta_{i}$ must be between $\beta_{m i n ; i}$ and $\beta_{m a x ; i}$, the upper and lower bounds for all components $i$

d) the reference time $t_{0}$, each acceleration factor $A F_{i j}$ and all Weibull parameters $\beta_{i}$ must not be negative

e) the confidence level $b$ must be between $0 \%$ and $100 \%$

f) each test is either counted for a certain component or is obsolete

The function for $B_{X}$ - lifetime is closely related to the reliability function since it is the inversion of the CDF. Therefore the optimization model for $B_{X}$ - lifetime can be set up in a comparable way. NLP (5.2) is the respective model for the $b$ LCL of $B_{X}$ - lifetime $B_{X ; S ; L C L ; b}$, that considers the uncertainty of $\beta_{i}$.

Where:

a) calculation of the objective, $B_{X ; S ; L C L ; b}$, according to the second theorem (3.17).

b) calculation of the Weibull equivalent single test duration $T W_{i}$ according to (3.5) for all components $i$ (like NLP (5.1))

c) $\beta_{i}$ must be between $\beta_{\text {min;i }}$ and $\beta_{\max ; i}$, the upper and lower bounds for all components $i$ (like NLP (5.1))

d) each acceleration factor $A F_{i j}$ and all Weibull parameters $\beta_{i}$ must not be negative

e) the confidence level $b$ must be between $0 \%$ and $100 \%$ (like NLP (5.1))

f) $X$, the share of system that fail up to duration $B_{X}$

g) each test is either counted for a certain component or is obsolete (like NLP (5.1)) 
Minimize $\quad B_{X ; S ; L C L ; b}$

subject to

a) $B_{X ; S ; L C L ; b}=\operatorname{Min}_{i}\left[\left(\frac{\ln \left(1-\frac{X \%}{100 \%}\right) * T W_{i}^{\beta_{i}}}{\ln (b)}\right)^{1 / \beta_{i}}\right]$

b) $T W_{i}=\sqrt[\beta_{i}]{\sum_{j=1}^{m}\left(t_{j} * A F_{i j} * o_{i j}\right)^{\beta_{i}}} \quad$ for $i=1, . ., n$

c) $\beta_{\text {min } ; i} \leq \beta_{i} \leq \beta_{\max ; i} \quad$ for $i=1, \ldots, n$

d) $A F_{i j} ; \beta_{i} \geq 0 \quad$ for $i=1, . ., n ;$ for $j=1, . ., m$

e) $0 \leq b \leq 1$

f) $0 \leq X \leq 100$

g) $o_{i j}=[0 ; 1] \quad$ for $i=1, . ., n ;$ for $j=1, \ldots, m$ 
The NLP (5.2) for $B_{X}$ - lifetime, like the model for system reliability, is a minimization with the $\beta_{i}$ being the decision variables. The parameters are very similar to those in (5.1) but include the share $X$ instead of the duration $t_{0}$. So the parameters of the NLP (5.2) for $B_{X}$ are:

- information about the conducted reliability tests: test durations $t_{j}$, acceleration factors $A F_{i j}$, boolean variables to indicate the test relevance $o_{i j}$

- upper and lower bound for $\beta_{i}: \beta_{\min ; i}$ and $\beta_{\max ; i}$

- maximum share of system that are allowed to fail: $X$

- confidence level $b$

\subsection{Application of the Model to Consider $\beta$ Sensitivity (Example)}

Here we apply the two models to the example that was introduced in Chapter 3. In Chapter 3 the Weibull shape parameters $\beta_{i}$ for each component $i$ were fixed values. Now the example is extended, such that $\beta_{i}$ is a variable and $\beta_{\max , i}$ and $\beta_{\min , i}$ are the upper and lower bounds for each component Weibull parameter $\beta_{i}$. Table 5.1 compares the fixed values for $\beta_{i}$ to the upper and lower bound:

\begin{tabular}{|c|c|c|c|c|c|}
\hline$\beta \_$min & 1.3 & 1.75 & 1.5 & 1.25 & 0.8 \\
\hline$\beta \_$max & 1.7 & 2.3 & 2 & 1.35 & 1.2 \\
\hline \hline$\beta \_$fixed & 1.5 & 2 & 1.8 & 1.3 & 1 \\
\hline
\end{tabular}

Table 5.1 Comparison of fixed values for $\beta_{i}$ vs. upper and lower bound 
The NLP 5.1 is programmed in LINGO $^{\mathrm{TM}}$, an universal solver package. Since the objective function is discontinuous including a minimum-operation, the global solver of LINGO $^{\mathrm{TM}}$ was used to prevent stops after finding solutions that are only locally (but not globally) optimal. The software package and the computer hardware used in the analysis is specified in Appendix 6.

\section{System Reliability}

NLP (5.1) is a minimization and therefore any increase in the permitted area for the decision can only lead to the same or lower values for the objective function. So in any case the $b$ LCL of system reliability will be lower (or equal) than the value determined with fixed values for the component $\beta_{i}$ as long as the fixed value is a part of the interval between lower and upper bound. Figure 5.1 compares the resulting $b$ LCL of system reliability for both cases fixed and variable component $\beta_{i}$.

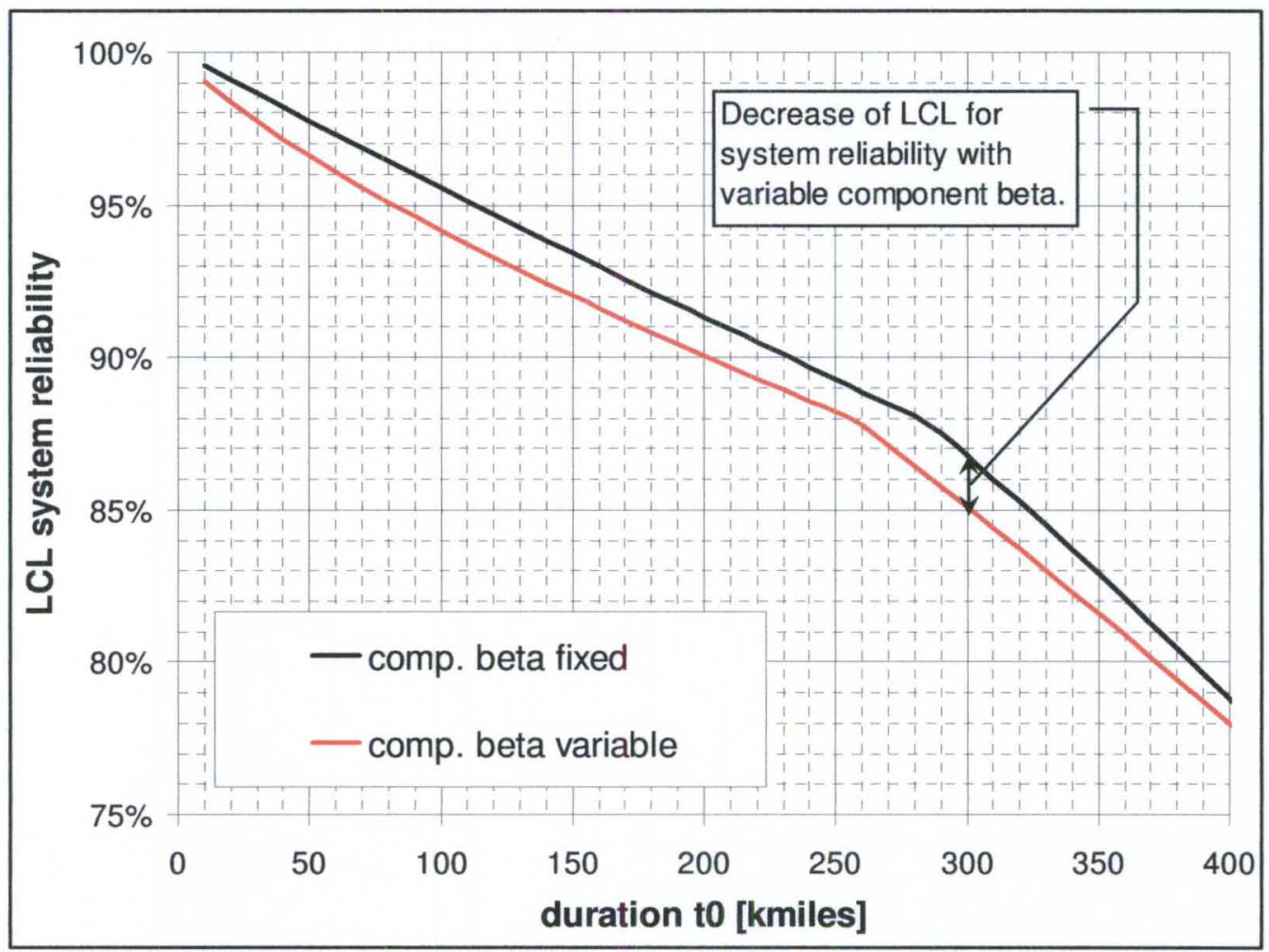

Figure 5.1 Comparison of $b$ LCL of system reliability with fixed and variable $\beta_{i}$ 


\begin{tabular}{|c|c|c|c|c|c|c|c|c|}
\hline 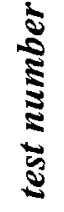 & 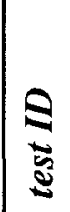 & 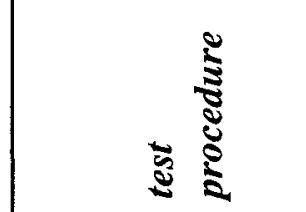 & 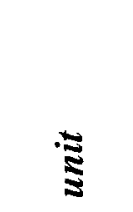 & 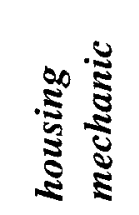 & 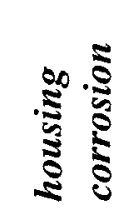 & 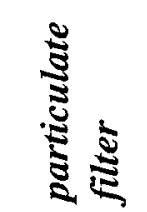 & 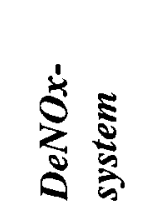 & 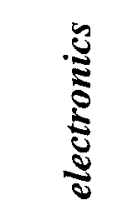 \\
\hline & & & & \multicolumn{5}{|c|}{ effective mileage (per component) } \\
\hline 1 & $A$ & hydropuls test & kmiles & 0 & 0 & 0 & 0 & 0 \\
\hline 2 & $H$ & spray test & kmiles & 0 & 2250 & 0 & 750 & 750 \\
\hline 3 & $A$ & hydropuls test & kmiles & 750 & 0 & 0 & 0 & 0 \\
\hline 4 & $A$ & hydropuls test & kmiles & 750 & 0 & 0 & 0 & 0 \\
\hline 5 & $H$ & spray test & kmiles & 750 & 225 & 0 & 750 & 750 \\
\hline 6 & $F$ & customer testing & kmiles & 200 & 200 & 0 & 200 & 200 \\
\hline 7 & $G$ & alternating load & kmiles & 0 & 0 & 600 & 0 & 600 \\
\hline 8 & $H$ & spray test & kmiles & 750 & 225 & 750 & 750 & 750 \\
\hline 9 & $I$ & WHTC cycle & kmiles & 0 & 150 & 0 & 450 & 300 \\
\hline 10 & $B$ & corrosion test & kmiles & 0 & 750 & 0 & 0 & 0 \\
\hline 11 & $D$ & city cycle & kmiles & 100 & 300 & 300 & 300 & 200 \\
\hline 12 & $E$ & rough road test & kmiles & 0 & 21 & 21 & 21 & 21 \\
\hline 13 & $D$ & city cycle & kmiles & 100 & 300 & 300 & 300 & 200 \\
\hline 14 & $C$ & express-run & kmiles & 200 & 200 & 300 & 300 & 200 \\
\hline 15 & $I$ & WHTC cycle & kmiles & 0 & 150 & 450 & 450 & 300 \\
\hline 16 & $C$ & express-run & kmiles & 200 & 200 & 300 & 300 & 200 \\
\hline 17 & $F$ & customer testing & kmiles & 200 & 200 & 200 & 200 & 200 \\
\hline 18 & $F$ & customer testing & kmiles & 200 & 200 & 200 & 200 & 200 \\
\hline 19 & $F$ & customer testing & kmiles & 200 & 200 & 200 & 200 & 200 \\
\hline \multicolumn{3}{|c|}{ lower bound $\beta$} & $\beta \_\min$ & 1.3 & 1.75 & 1.5 & 1.25 & 0.8 \\
\hline \multicolumn{3}{|r|}{ upper bound $\beta$} & $\beta \_\max$ & 1.7 & 2.3 & 2 & 1.35 & 1.2 \\
\hline \multicolumn{3}{|r|}{ Duration to } & kmiles & \multicolumn{5}{|c|}{400} \\
\hline \multicolumn{3}{|r|}{ Conf. Level $b$} & - & \multicolumn{5}{|c|}{0.10} \\
\hline \multicolumn{3}{|c|}{$\beta$ for $L C L$ system $R$} & $\beta \_L C L$ & 1.30 & 1.75 & 1.50 & 1.25 & 0.85 \\
\hline \multicolumn{3}{|c|}{$T W_{-} i$} & kmiles & 2674 & 2666 & 1765 & 3189 & 8014 \\
\hline \multicolumn{3}{|r|}{$R \_L C L \_C(t 0)$} & $\%$ & $82.30 \%$ & $92.01 \%$ & $78.00 \%$ & $84.21 \%$ & $83.32 \%$ \\
\hline \multicolumn{3}{|r|}{$R \_C L \_S(t 0)$} & $\%$ & \multicolumn{5}{|c|}{$78.00 \%$} \\
\hline
\end{tabular}

Table 5.2 LCL of system reliability at $t_{0}=400$ with variable and fixed $\beta_{i}$ 
Table 5.2 compares in detail the calculation of the $b$ LCL of system reliability with variable and fixed component $\beta_{i}$ at duration $t_{0}=400$. In both cases, fixed and variable $\beta_{i}$, component 3 , the particulate filter, is the limiting component for the LCL of system reliability. Compared to the scenario with a fixed Weibull parameter of this component $\beta_{3, \text { fixed }}=1.8$ the NLP finds its minimum for $\beta_{3, \min }=1.5$ which is exactly the lower bound of this component Weibull parameter. The lower total knowledge in the system behavior leads to a reduction of the $b \mathrm{LCL}$ of system reliability from $78.79 \%$ to $78.00 \%$ at the considered duration $t_{0}=400$ kmiles.

\section{$\underline{\text { System }} \mathrm{B}_{\mathrm{X}}$ - lifetime}

Like the system reliability, the $b$ LCL of $B_{X}$ - lifetime is also reduced if there is a range for component $\beta_{i}$ instead of fixed values within the respective range. The respective values of the $B_{X}$ - lifetime can be calculated with NLP (5.2). Table 5.3 shows that with the ranges that were introduced in Table 5.1 there is a reduction of the $b \mathrm{LCL}$ of $B_{10}$ - lifetime from 232 kmiles to 201 kmiles. 


\begin{tabular}{|c|c|c|c|c|c|c|c|c|}
\hline 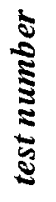 & $\begin{array}{l}8 \\
\text { vas }\end{array}$ & 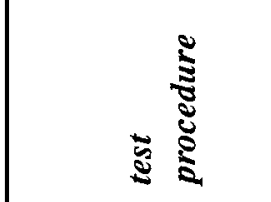 & $\stackrel{\vec{\Xi}}{\mathbf{\Xi}}$ & 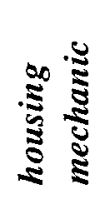 & $\begin{array}{r}5 \\
5 \\
5 \\
5 \\
5 \\
5 \\
5 \\
5 \\
5\end{array}$ & 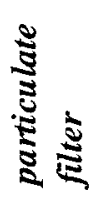 & 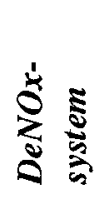 & 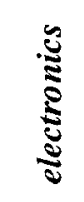 \\
\hline & & & & \multicolumn{5}{|c|}{ effective mileage (per component) } \\
\hline 1 & $A$ & hydropuls test & kmiles & 0 & 0 & 0 & 0 & 0 \\
\hline 2 & $H$ & spray test & kmiles & 0 & 2250 & 0 & 750 & 750 \\
\hline 3 & $A$ & hydropuls test & kmiles & 750 & 0 & 0 & 0 & 0 \\
\hline 4 & $A$ & hydropuls test & kmiles & 750 & 0 & 0 & 0 & 0 \\
\hline 5 & $H$ & spray test & kmiles & 750 & 225 & 0 & 750 & 750 \\
\hline 6 & $F$ & customer testing & kmiles & 200 & 200 & 0 & 200 & 200 \\
\hline 7 & $G$ & alternating load & kmiles & 0 & 0 & 600 & 0 & 600 \\
\hline 8 & $H$ & spray test & kmiles & 750 & 225 & 750 & 750 & 750 \\
\hline 9 & $I$ & WHTC cycle & kmiles & 0 & 150 & 0 & 450 & 300 \\
\hline 10 & $B$ & corrosion test & kmiles & 0 & 750 & 0 & 0 & 0 \\
\hline 11 & $D$ & city cycle & kmiles & 100 & 300 & 300 & 300 & 200 \\
\hline 12 & $E$ & rough road test & kmiles & 0 & 21 & 21 & 21 & 21 \\
\hline 13 & $D$ & city cycle & kmiles & 100 & 300 & 300 & 300 & 200 \\
\hline 14 & $\bar{C}$ & express-run & kmiles & 200 & 200 & 300 & 300 & 200 \\
\hline 15 & $I$ & WHTC cycle & kmiles & 0 & 150 & 450 & 450 & 300 \\
\hline 16 & $C$ & express-run & kmiles & 200 & 200 & 300 & 300 & 200 \\
\hline 17 & $F$ & customer testing & kmiles & 200 & 200 & 200 & 200 & 200 \\
\hline 18 & $F$ & customer testing & kmiles & 200 & 200 & 200 & 200 & 200 \\
\hline 19 & $F$ & customer testing & kmiles & 200 & 200 & 200 & 200 & 200 \\
\hline \multicolumn{3}{|c|}{ lower bound $\beta$} & $\beta \_$min & 1.3 & 1.75 & 1.5 & 1.25 & 0.8 \\
\hline \multicolumn{3}{|c|}{ upper bound $\beta$} & $\beta \_\max$ & 1.7 & 2.3 & 2 & 1.35 & 1.2 \\
\hline \multicolumn{3}{|c|}{ share of units failed $X$} & $\%$ & \multicolumn{5}{|c|}{10} \\
\hline \multicolumn{3}{|c|}{ Conf. Level b } & - & \multicolumn{5}{|c|}{0.10} \\
\hline \multicolumn{3}{|r|}{$\beta$ for $L C L B x$} & $\beta \_L C L$ & 1.3 & 1.75 & 1.5 & 1.25 & 0.8 \\
\hline \multicolumn{3}{|r|}{$T W_{-} i$} & kmiles & 2674 & 2666 & 1765 & 3189 & 9516 \\
\hline \multicolumn{3}{|r|}{ var. $\beta B x_{-} C$} & kmiles & 249 & 457 & 226 & 270 & 201 \\
\hline \multicolumn{3}{|r|}{ var. $\beta B x_{-} S$} & kmiles & \multicolumn{5}{|c|}{201} \\
\hline \multicolumn{3}{|r|}{$\beta$ fixed } & $\beta \_L C L$ & 1.5 & 2 & 1.8 & 1.3 & $I$ \\
\hline \multicolumn{3}{|r|}{$T W_{-} i$} & kmiles & 2171 & 2488 & 1410 & 2965 & 5071 \\
\hline \multicolumn{3}{|r|}{ fixed $\beta B x_{-} C$} & kmiles & 278 & 532 & 254 & 276 & 232 \\
\hline & & fixed $\beta B x_{-} S$ & kmiles & \multicolumn{5}{|c|}{232} \\
\hline
\end{tabular}

Table 5.3 LCL of $B_{X}$ - lifetime $(X=10)$ with variable and fixed $\beta_{i}$ 
Variation of $\underline{\beta}$

Figure 5.2 shows that for the duration $t_{0}=400 \mathrm{kmiles}$, there is a single value of the Weibull parameter $\beta_{o p t, i}$ which leads to a minimum of the component reliability $R_{C ; i ; L C L ; b}$. If $\beta_{o p t, i}$ is not within the permitted range of $\beta_{i}$, i.e. $\beta_{o p t, i}<\beta_{\min , i}$ or $\beta_{o p t, i}>\beta_{\max , i}$ respectively, the minimum is reached either for $\beta_{\min , i}$ or for $\beta_{\max , i}$, whichever of these two is closer to $\beta_{\text {opt }, i}$.

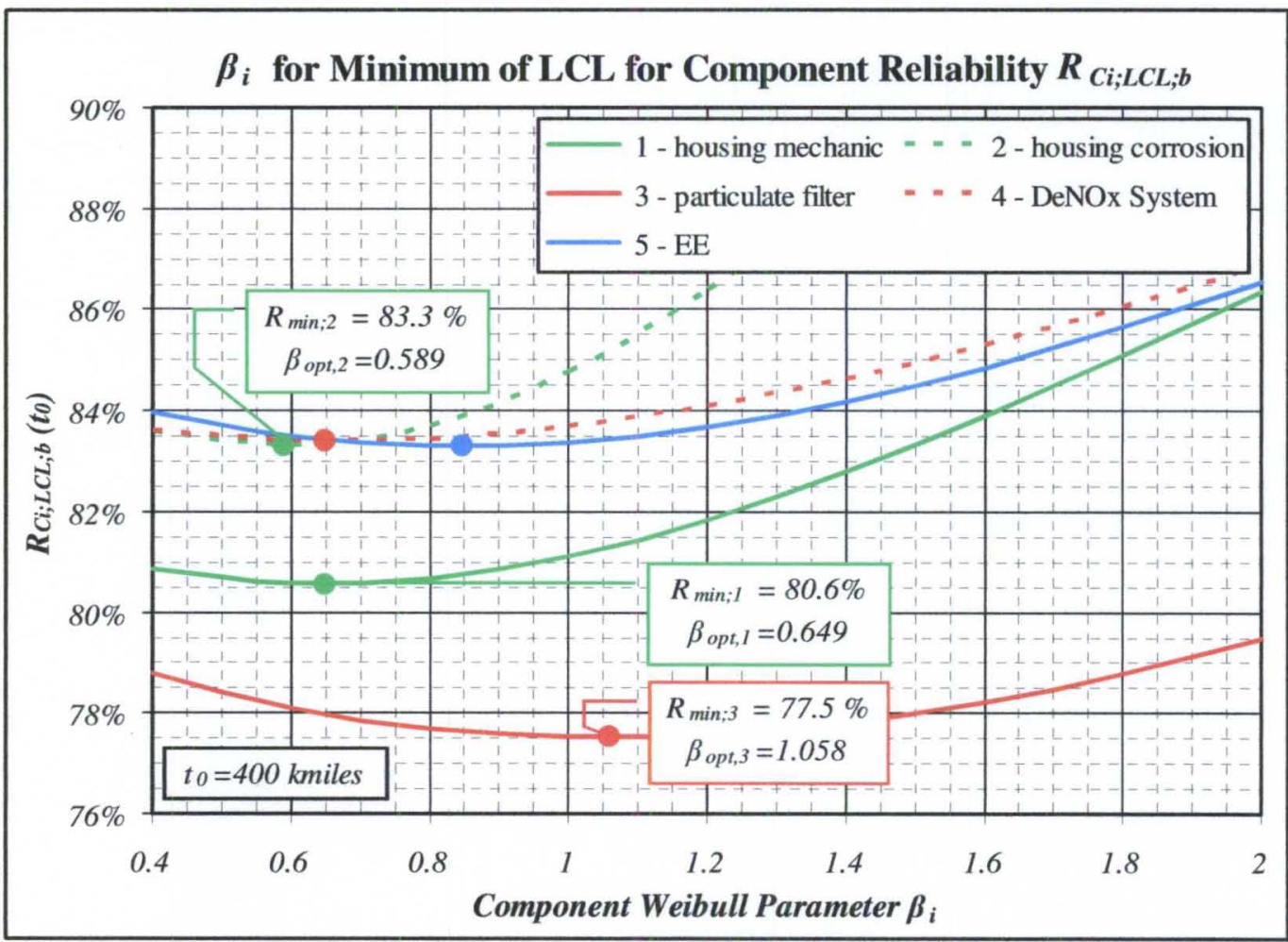

Figure 5.2 Minimum of $b$ LCL of system reliability with unrestricted $\beta_{i}$

If there is no limiting constraint on $\beta_{i}$ (except to non-negativity) the $b$ LCLs of the component reliability of all components $i$ are exactly at the respective minimum $\beta_{\text {opt }, i}$. The $b$ LCL of system reliability is then the lowest value of the LCLs of reliability at $\beta_{o p t, i}$ among all components $i$ as shown in the Table 5.4. For the given example this results in $R_{S ; L C L ; b}=77.53 \%$ at the duration $t_{0}=400$ kmiles and for $b=0.1$. The comparable value with the previously assumed fixed values for the Weibull parameter $\beta_{i}$ at the same duration is $78.79 \%$ (see Table 3.4 ). 


\begin{tabular}{|c|c|c|c|c|c|}
\hline 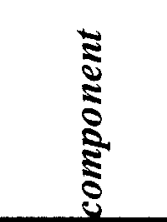 & 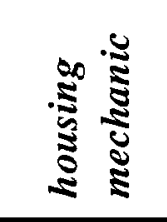 & 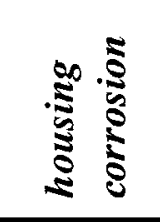 & 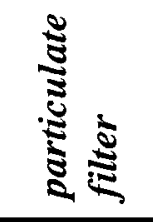 & 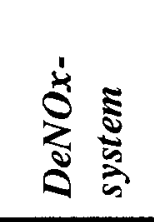 & 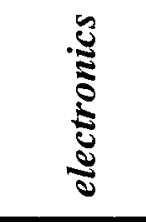 \\
\hline$\beta_{o p t ; i}$ & 0.649 & 0.589 & 1.058 & 0.649 & 0.846 \\
\hline$R_{C i ; L C ; b}$ & $80.56 \%$ & $83.32 \%$ & $77.53 \%$ & $83.40 \%$ & $83.32 \%$ \\
\hline$R_{S ; L C ; b}$ & \multicolumn{5}{|c|}{$77.53 \%$} \\
\hline
\end{tabular}

Table 5.4 $\quad b=0.1 \mathrm{LCL}$ of system reliability $\left(t_{0}=400\right)$ with unrestricted $\beta_{i}$

\subsection{Remark on Efficient Optimization Model Solution}

NLP (5.1) for system reliability or (5.2) for $B_{X}$ - lifetime respectively are highly non-linear. The objective function is discontinuous since it includes a minimumoperation. Together, this leads to a very complex optimization problem with long processing times. Solving the NLPs directly as defined in equation system (5.1) or (5.2) can lead to very long solution times if a universal solver software package like the general solver of $\mathrm{LINGO}^{\mathrm{TM}}$ is applied. Therefore, the following strategy is proposed to reduce the processing time significantly:

- Find the Weibull parameter $\beta_{o p t, i}$ for each component $i$ that leads to the minimum of the LCL of reliability or $B_{X}$ - lifetime of the respective component. This optimization, with just one variable at a time, can be solved within seconds using an adequate solver software package.

- Calculate the LCLs of component reliability or component $B_{X}$ - lifetime for the value of the Weibull parameter $\beta_{o p t, i}$ that was determined in the step before.

- Compare the LCLs of component reliability or component $B_{X}$ - lifetime. The minimum of them is the LCL of system reliability or the LCL of system $B_{X}$ - lifetime. 


\section{OPTIMIZATION OF RDT PROGRAMS}

\subsection{Relevant Cost Information for Decision Making}

Relevant information for decision making is characterized by the following attributes ${ }^{19}$ :

- the information is related to future cost or revenue

- the information must allow to distinguish between two or more alternatives

Thus information concerning cost that have already been spent in the past and thus can no longer be influenced, i.e. sunk cost, is not relevant for decision making. Optimization of the RDT program is considered to be a rolling planning process. At each moment during the RDT program, the reliability already demonstrated can be calculated considering the accumulated mileage and the failures that have occurred. As mentioned before, the cost spent in the past to achieve this level of reliability demonstration is not relevant, with respect to optimizing future RDT activities. Only the future spending for RDT that still can be influenced is relevant for the decision making process.

For the optimization of the future RDT program, the available test procedures must be known, including information about test cost, mileage and the component specific stress factors. All of this information is documented in a test procedure database. It includes test information such as:

- fixed and variable costs for a given test type

- minimum and maximum permitted sample sizes for a given test type

- minimum and maximum test durations for a given test type

\footnotetext{
${ }^{19}$ see: Horngreen, Sundem, Stratton, Burgstahler and Schatzberg (2008), p. 198
} 
Based on this information, different RDT scenarios can be described and evaluated in terms of the associated cost and the reliability level that can be demonstrated. The purpose of the optimization is to find out the best RDT plan to meet the reliability targets in the most efficient way or to achieve the highest reliability level within the given constraints on budget or prototype availability.

\subsection{Model for Confidence Limit of Total Cost Influenced by Validation}

As mentioned in Chapter 2.6 the total product cost over the lifecycle can be influenced by the extent of validation:

- More effort spent in validation leads to higher reliability and thus less warranty cost.

- Less effort in validation reduces the required overall budget for validation and development but increases the warranty cost.

The relationship between the extent of reliability on the one hand and reliability and warranty cost on the other hand is not clear - at least not in the phase in which the decision on the RDT program has to be made.

The upper part of Figure 6.1 shows the increasing LCL of reliability depending on the extent of testing. This relationship is qualitatively true for single items as well as for systems. For each length of testing it is not clear what the real value of reliability actually is. It is only clear that with the confidence level $b$ the real value is higher than the LCL. All efficient RDT programs are on a line that represents solutions of the NLP that will be

introduced in the next section. Any RDT program that leads to a LCL of reliability below the marked area is not efficient in terms of creating the highest contribution to reliability demonstration with a certain consumption of resources. 
The warranty cost (the cost of unreliability) can be calculated by multiplying the cost per failure and the reliability which reflects the frequency of failure in the field and the total production volume. Of course, the cost per failure depends on the actual failure mode. For systems, the cost of a each failure depends also from on the affected component of the system. But for reasons of simplicity it is assumed that all types of failures create the same cost per incident.

Assuming a constant cost per failure, the LCL of reliability (which is a lower limit) can be transformed into an upper confidence limit (UCL) on warranty cost. With an increasing extent of validation testing the reliability gets higher, and we see a reduction of the UCL of warranty cost. The cost for validation is typically proportional to the extent of validation testing. The cost curve for validation and the possible area of the warranty cost limited by the UCL are shown in the middle diagram of Figure 6.1.

The sum of both, the cost for validation and the warranty cost, yields a sum of total cost that can be influenced by validation (depending on the extent of validation testing). The area of this sum at each validation level goes from the respective validation cost to the upper confidence limit of warranty plus validation cost. This is illustrated in the lower part of Figure 6.1.

The UCL of the total cost has a minimum value. If possible, it is recommended to test to exactly this point. Of course the actual reliability can be higher than the LCL. In this case the associated warranty cost is lower and it would have economical advantages if the testing effort was reduced. But such a decision can put the profitability of the product at risk since it is not clear from the very beginning if reliability really is higher than the LCL. 

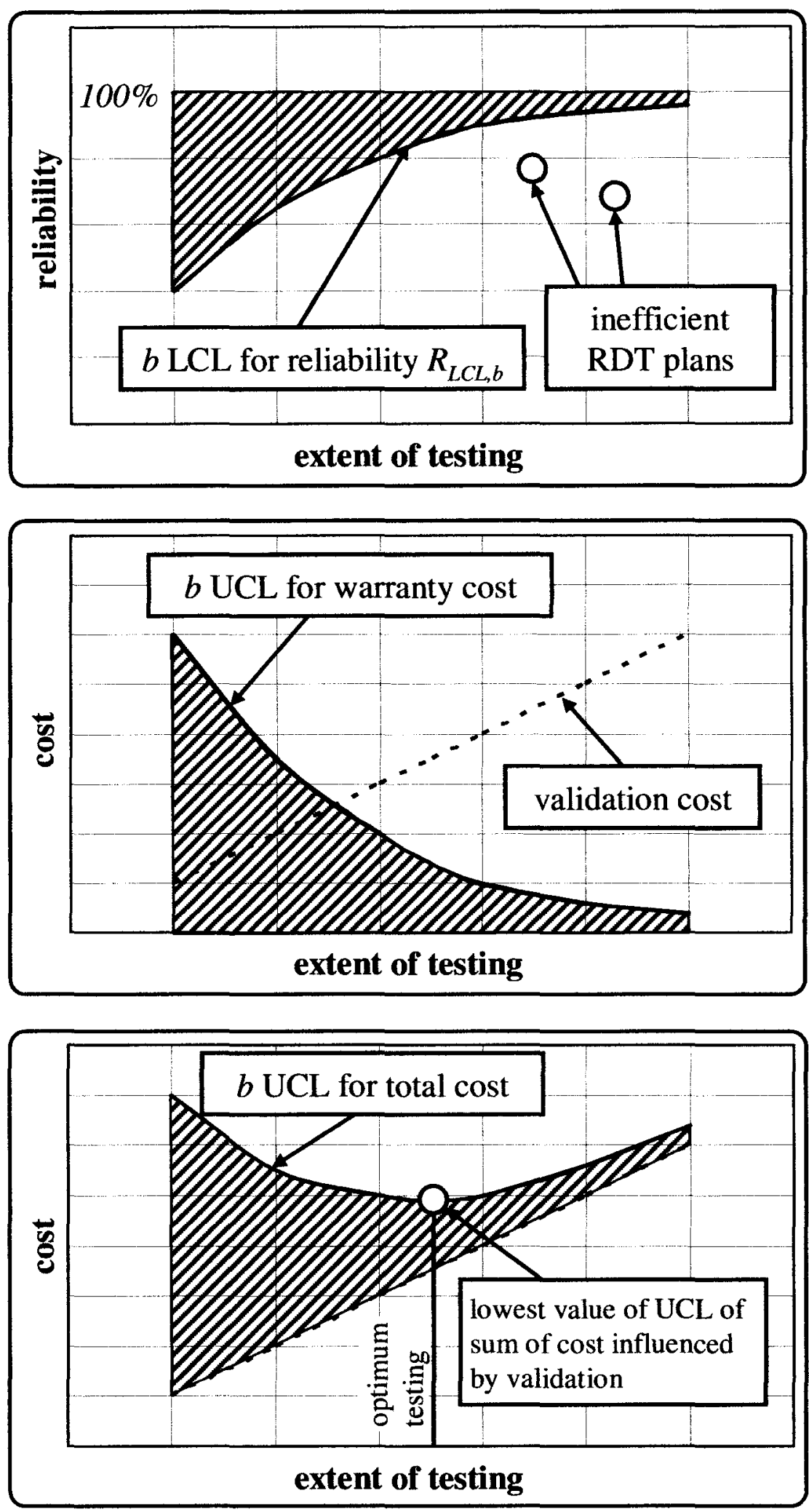

Figure 6.1 LCL of reliability and UCL for warranty and total cost 


\subsection{Model for Optimizing RDT Plans with Respect to the LCL of Reliability}

All efficient RDT plans lead to the highest possible level of the system reliability for the given budget constraints. This means that they maximize the LCL of system reliability within the given constraints of the validation budget or limitations concerning minimum and maximum test durations or sample sizes for a certain test type. The optimization model is a highly non-linear mixed-integer NLP. The variable $i(i=1 . . n)$ denotes a component. Tests that are already finished are indicated with the variable $j\left(j=1 . . m_{F}\right)$ and the index $F$. Future test, that are so far only planned, are denoted with the variable $k$ ( $\left.k=1 . . m_{P}\right)$ and the index $P$.

The decision variables of the optimization problem are:

- duration of the planned test according to test type $k: t_{P k}$

- $\quad$ sample size for the test according to test type $k: l_{P k}$

The parameters of the NLP are:

- duration (i.e. reference duration for the statement on system reliability): $t_{0}$

- confidence level: $b$

- component Weibull shape parameter of component $i: \beta_{i}$

- information about the already finished reliability tests:

test durations $t_{F i j}$ that indicate the relevant test time of test $j$ for component $i$ (remark: $t_{F i j}$ already considers acceleration factors $A F_{F i j}$ and the Boolean variable $o_{F i j}$ for indication if the test is obsolete or relevant for component $i$ )

- lower and upper limit for the test duration and sample size according to test type $k: t_{P, \min k}$ and $t_{P, \max k}, l_{P, \min k}$ and $l_{P, \max k}$

- calculatory speed to transform the duration of test $k$ from a hourly base into a vehicle mileage equivalent: $v_{P k}$

- acceleration factor of test $k$ for component $i: A F_{P i k}$

- fixed cost of one test according to test type $k: C_{f k}$ 
- variable cost of test type $k$ for one unit of test duration (time or mileage): $C_{v k}$

- maximum available validation budget for all future tests: $C_{t \max }$

The NLP to determine the RDT plan with optimal system reliability is:

Maximize $R_{S ; L C L ; b}$

subject to

a) $R_{S ; L C L ; b}\left(t_{0}\right)=\operatorname{Min}\left[b^{\left(\frac{t_{0}^{\beta_{i}}}{T W_{i}^{\beta_{i}}}\right)}\right]$

b) $T W_{i}^{\beta_{i}}=\sum_{j=1}^{m_{F}} t_{F i j} \beta_{i}$

$$
+\sum_{k=1}^{m_{P}}\left[l_{P k} *\left(t_{P k} * v_{P k} * A F_{P i k}\right)^{\beta_{i}}\right] \quad \text { for } i=1, \ldots, n
$$

c) $C_{t k}=l_{P k} *\left(C_{f k}+C_{v k} * t_{P k}\right) \quad$ for $k=1, . ., m_{P}$

d) $\sum_{k=1}^{m_{P}} C_{t k} \leq C_{t \max }$

e) $t_{P, \min k} \leq t_{P k} \leq t_{P, \max k} \quad$ for $k=1, \ldots, m_{P}$

f) $l_{P, \min k} \leq l_{P k} \leq l_{P, \max k} \quad$ for $k=1, \ldots, m_{P}$

g) $l_{P k}=[0 ; 1 ; 2 ; 3 ; \ldots .$.$] for k=1, \ldots, m_{P}$

h) $t_{0} ; A F_{P i k} ; v_{P k} ; \beta_{i} \geq 0 \quad$ for $i=1, . ., n ;$ for $k=1, . ., m_{P}$

i) $0 \leq b \leq 1$ 
Where:

a) calculation of the objective, $R_{S ; L C L ; b}$, according to the first theorem (3.15).

b) calculation of the Weibull equivalent single test duration $T W_{i}$ according to (3.5) for all components $i$ considering completed and future tests

c) calculation of the total cost (variable and fixed costs) for all tests $k$

d) constraint that the grand total cost of all test costs is within the budget

e) all tests $k$ meet the constraints on minimum and maximum duration

f) all tests $k$ meet the constraints on minimum and maximum sample size

g) the sample size of all tests $k$ are integer

h) the reference time $t_{0}$, each acceleration factor $A F_{i j}$, the calculatory speed $v_{P k}$ of each test $k$ and all Weibull parameters $\beta_{i}$ must not be negative

i) the confidence level $b$ must be between $0 \%$ and $100 \%$

All the solutions of the NLP (6.1) for a certain validation budget are optimal RDT plans since they all lead to the maximum LCL of system reliability within the existing constraints. The respective points of each solution of the NLP lead to the curve that limits the marked area in the upper diagram of Figure 6.1.

The NLP of (6.1) is very complex to solve since it includes operations to the power of $\beta_{i}$ as well as operations to the power of $\left(1 / \beta_{i}\right)$. This requires significant solver processing times using a universal solver like $\mathrm{LINGO}^{\mathrm{TM}}$, even for the case of only $n=5$

components. Due to the minimum operation the optimization within LINGO $^{\mathrm{TM}}$ has to be done with the "global solver". Other although faster solvers algorithms face the risk of only finding local optimal solutions. 


\subsection{Application of the RDT Optimization Model (example)}

The application of the model is illustrated in the example given about exhaust gas

aftertreatment systems that was introduced before. Table 6.1 shows the documentation of tests that are already finished. The respective times of certain tests are different for the various components since the test were performed including component specific acceleration factors. The component Weibull shape parameters $\beta_{i}$ and the confidence level $b$ are also given in Table 6.1. All reliability statements are related to the duration $t_{0}=400$ kmiles.

\begin{tabular}{|c|c|c|c|c|c|c|c|}
\hline 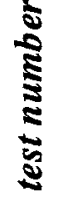 & 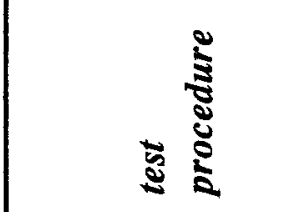 & $\stackrel{\mathbb{\Xi}}{\mathbf{\Xi}}$ & 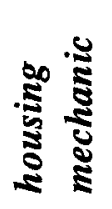 & 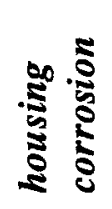 & 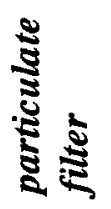 & $\sum_{\Delta}^{\vdots}$ & 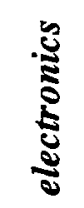 \\
\hline & & & \multicolumn{5}{|c|}{ effective mileage (per component) } \\
\hline 1 & hydropuls test & kmiles & 0 & 0 & 0 & 0 & 0 \\
\hline 2 & spray test & kmiles & 0 & 2250 & 0 & 750 & 750 \\
\hline 3 & hydropuls test & kmiles & 750 & 0 & 0 & 0 & 0 \\
\hline 4 & hydropuls test & kmiles & 750 & 0 & 0 & 0 & 0 \\
\hline 5 & spray test & kmiles & 750 & 225 & 0 & 750 & 750 \\
\hline 6 & customer testing & kmiles & 200 & 200 & 0 & 200 & 200 \\
\hline 7 & alternating load & kmiles & 0 & 0 & 600 & 0 & 600 \\
\hline 8 & spray test & kmiles & 750 & 225 & 750 & 750 & 750 \\
\hline 9 & WHTC cycle & kmiles & 0 & 150 & 0 & 450 & 300 \\
\hline 10 & corrosion test & kmiles & 0 & 750 & 0 & 0 & 0 \\
\hline 11 & city cycle & kmiles & 100 & 300 & 300 & 300 & 200 \\
\hline 12 & rough road test & kmiles & 0 & 21 & 21 & 21 & 21 \\
\hline 13 & city cycle & kmiles & 100 & 300 & 300 & 300 & 200 \\
\hline 14 & express-run & kmiles & 200 & 200 & 300 & 300 & 200 \\
\hline 15 & WHTC cycle & kmiles & 0 & 150 & 450 & 450 & 300 \\
\hline 16 & express-run & kmiles & 200 & 200 & 300 & 300 & 200 \\
\hline 17 & customer testing & kmiles & 200 & 200 & 200 & 200 & 200 \\
\hline 18 & customer testing & kmiles & 200 & 200 & 200 & 200 & 200 \\
\hline 19 & customer testing & kmiles & 200 & 200 & 200 & 200 & 200 \\
\hline & Veibull Parameter & $\beta$ & 1.5 & 2.0 & 1.8 & 1.3 & 1.0 \\
\hline & Duration t0 & kmiles & 400 & & & & \\
\hline & Conf. Level b & - & 0.10 & & & & \\
\hline
\end{tabular}

Table 6.1 RDT optimization: documentation of already finished tests 
The durations of the completed tests of Table 6.1 can be transformed to the Weibull equivalent single test durations $T W_{F i}$ for all components $i$ with equation (3.16) and the $b$ LCL of component reliability at $t_{0}$ as shown in the Table 6.2. Applying the first theorem of (3.15) the test data lead to the $b$ LCL of system reliability $R_{S ; L C L ; b}=78.89 \%$ at $t_{0}=400$ kmiles with the particulate filter being the limiting component.

\begin{tabular}{|c|c|c|c|c|c|c|}
\hline$T W_{F i}$ of finished tests & kmiles & 2170.6 & 2488.3 & 1410.2 & 2965.4 & 5071.0 \\
\hline$R \_L C L \_C i$ & - & $83.35 \%$ & $94.22 \%$ & $78.79 \%$ & $84.34 \%$ & $83.39 \%$ \\
\hline$R \_L C L \_S$ & - & $78.79 \%$ & \multicolumn{1}{|l}{} \\
\hline
\end{tabular}

Table 6.2 RDT optimization: $T W_{F i}$ and $R_{S ; L C L ; b}$ based on the finished tests

The demonstrated reliability level is not considered to be sufficient. So it is decided to run an additional RDT program. There is an additional validation budget of $\$ 5,000,000$ available for the extension of RDT. The set of possible RDT programs is listed in the test procedure database as shown in Table 6.3. A part of these tests is conducted on test benches and not in a vehicle. This leads to an hourly basis for the duration instead of a mileage basis Table 6.3 also includes the ALT acceleration factors for the different components in all test procedures.

\begin{tabular}{|c|c|c|c|c|c|c|c|}
\hline 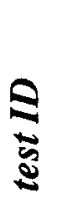 & 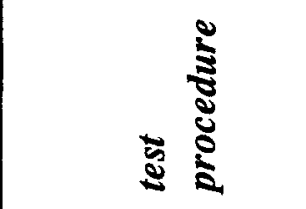 & $\stackrel{\stackrel{\vec{\Xi}}{\mathbf{Z}}}{ }$ & 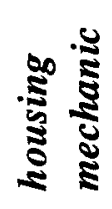 & 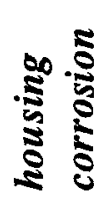 & 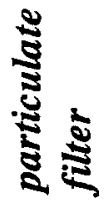 & 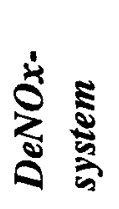 & 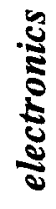 \\
\hline$A$ & hydropuls test & hours & 1 & 0 & 0 & 0 & 0 \\
\hline$B$ & corrosion test & hours & 0 & 1 & 0 & 0 & 0 \\
\hline$C$ & express-run & kmiles & 1 & 1 & 1.5 & 1.5 & 1 \\
\hline$D$ & city cycle & kmiles & 1 & 3 & 3 & 3 & 2 \\
\hline$E$ & rough road test & kmiles & 100 & 3 & 3 & 3 & 3 \\
\hline$F$ & customer testing & kmiles & 1 & $I$ & 1 & 1 & 1 \\
\hline$G$ & alternating load & hours & 0 & 0 & 2 & 2 & 2 \\
\hline$H$ & spray test & hours & 10 & 0.3 & 1 & 1 & 1 \\
\hline$I$ & WHTC cycle & hours & 0 & 0.5 & 1.5 & 1.5 & 1 \\
\hline
\end{tabular}

Table 6.3 RDT optimization: test procedure database - acceleration factors 
There are fixed and variable costs associated with the different test procedures as shown in the Table 6.4. The variable cost is related to one unit of the test duration. This unit is either 1 kmile or 1 hour. The tests that have an hourly base for the duration must be transformed into a vehicle mileage with the calculated speed conversion that is test specific.

There are constraints on the minimum and the maximum duration of each test. The upper limit of the test duration can be defined for example by the time left to the planned release and launch of the respective product. Other limitations are related to the minimum and maximum sample size for the different test procedures. Possible reasons for a lower limit of the sample size for a certain test type are needs for certain tests procedures besides RDT resulting from the DVP\&R-plan. The upper limit of sample size can be, for example, driven by the maximum capacity to operate prototype vehicles. In addition to the limitations on the sample size per test there is also a limitation of the total sample size of all tests to thirty units due to prototype building capacity restrictions.

\begin{tabular}{|c|c|c|c|c|c|c|c|c|c|}
\hline$\frac{8}{3}$ & 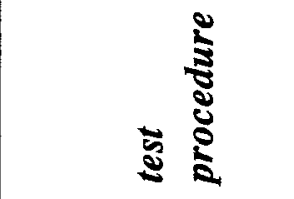 & 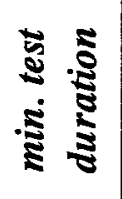 & 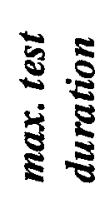 & $\stackrel{5}{5}$ & 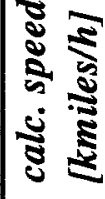 & 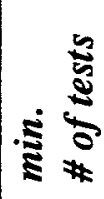 & 苛 & 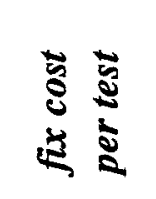 & 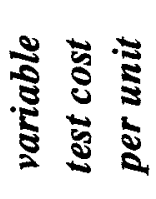 \\
\hline $\bar{A}$ & hydropuls test & 50 & 300 & hours & 5 & $\overline{0}$ & 5 & 80,000 & 300 \\
\hline $\bar{B}$ & corrosion test & 100 & 2000 & hours & 1 & 0 & 5 & 50,000 & 30 \\
\hline $\bar{C}$ & express-run & 30 & 300 & kmiles & 1 & $\overline{0}$ & 10 & 150,000 & 1,250 \\
\hline$D$ & city cycle & 10 & 120 & kmiles & $\overline{1}$ & 0 & 10 & 150,000 & 2,500 \\
\hline$E$ & rough road test & 2 & 15 & kmiles & 1 & $\overline{0}$ & 10 & 150,000 & 5,000 \\
\hline$F$ & customer testing & 25 & 225 & kmiles & $I$ & 0 & 10 & 150,000 & 100 \\
\hline$G$ & alternating load & 250 & 4000 & hours & 0.15 & 0 & 5 & 80,000 & 200 \\
\hline $\bar{H}$ & spray test & 250 & 4000 & hours & 0.15 & $\overline{0}$ & 5 & 80,000 & 200 \\
\hline$I$ & WHTC cycle & 250 & 4000 & hours & 0.15 & 0 & 5 & 80,000 & 220 \\
\hline
\end{tabular}

Table 6.4 RDT optimization: test procedure database - constraints and cost

The NLP (6.1) is solved with LINGO ${ }^{\mathrm{TM}}$. Since the NLP contains a minimum operation it is necessary to apply the global solver to avoid a situation that the solver finds solutions that are only locally optimal. 
Considering the described information about test procedures and the constraints including the available validation budget of $\$ 5,000,000$, Table 6.5 shows the solution that maximizes the LCL of system reliability.

\begin{tabular}{|c|c|c|c|c|c|}
\hline 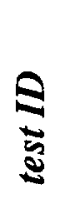 & 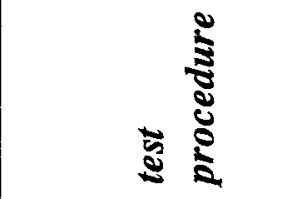 & క气 & $\stackrel{\Xi}{\mathbf{\Xi}}$ & $\frac{5}{5}$ & 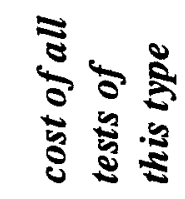 \\
\hline $\bar{A}$ & hydropuls test & 51 & hours & 0 & 0 \\
\hline$B$ & corrosion test & 101 & hours & 0 & 0 \\
\hline$C$ & express-run & 31 & kmiles & 0 & 0 \\
\hline$D$ & city cycle & 10 & kmiles & 0 & 0 \\
\hline$E$ & rough road test & 3 & kmiles & 0 & 0 \\
\hline$F$ & customer testing & 225 & kmiles & 1 & 172,500 \\
\hline$G$ & alternating load & 4,000 & hours & 5 & $4,400,000$ \\
\hline $\bar{H}$ & spray test & 1,738 & hours & 1 & 427,500 \\
\hline$I$ & WHTC cycle & 251 & hours & 0 & 0 \\
\hline
\end{tabular}

Table 6.5 RDT optimization: optimized validation program

The optimal solution includes the following tests:

- 1 unit on "customer testing" for the maximum duration duration of $225 \mathrm{kmiles}$

- 5 units on "alternating load test" for the maximum possible time of 4,000 hours

- I unit on "spray test" for the time of 1,738 hours

The following Table 6.6 shows the consequences of the additional testing on the LCL of system reliability with a value of $92.34 \%$ and electronics being the limiting component. The budget restriction with $\$ 5,000,000$ is a binding constraint whereas the limitation thirty additional tests is not binding. 


\begin{tabular}{|c|c|c|c|c|c|}
\hline & 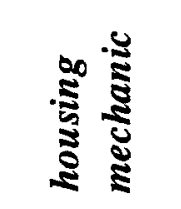 & 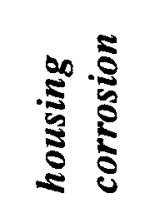 & 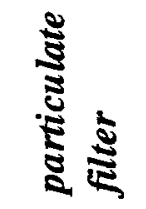 & 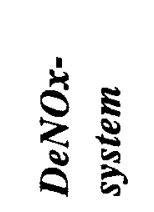 & 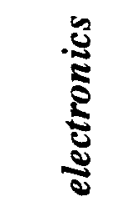 \\
\hline$R \_L C L \_C i$ & $92.54 \%$ & $94.27 \%$ & $95.18 \%$ & $93.71 \%$ & $92.34 \%$ \\
\hline Max. Number of Tests & 30 & & & & \\
\hline Max.Validation Budget & $5,000,000$ & & & & \\
\hline$R \_L C L \_S$ & $92.34 \%$ & & & & \\
\hline Add. Validation Cost & $5,000,000$ & & & & \\
\hline
\end{tabular}

Table 6.6 RDT optimization: achieved LCL of system reliability with add. testing

The optimization results for different budget constraints are shown in Table 6.7. With no additional testing the LCL of system reliability is $78.79 \%$. With an additional validation budget of $\$ 2,000,000$ the LCL of system reliability is significantly improved to $88.55 \%$, i.e. the complement of the reliability is almost cut into half. The additionally required effort to achieve even further improvements of the LCL of reliability is progressively increasing. With the validation budget of $\$ 20,875,000$ the LCL of system reliability then reaches its maximum value since above of this budget other constraints instead of the budget get limiting.

\begin{tabular}{|c|c|c|}
\hline $\begin{array}{c}\text { Add. Validation } \\
\text { Budget [\$] }\end{array}$ & R_LCL_S & $\begin{array}{c}\text { LINGO Solver } \\
\text { Time[h:m:s] }\end{array}$ \\
\hline 0 & $78.79 \%$ & $0: 00: 00$ \\
\hline 100,000 & $78.79 \%$ & $0: 00: 01$ \\
\hline 200,000 & $79.46 \%$ & $0: 00: 02$ \\
\hline 500,000 & $82.44 \%$ & $0: 00: 12$ \\
\hline $1,000,000$ & $86.31 \%$ & $0: 00: 47$ \\
\hline $2,000,000$ & $88.55 \%$ & $0: 03: 32$ \\
\hline $5,000,000$ & $92.34 \%$ & $0: 05: 56$ \\
\hline $10,000,000$ & $94.38 \%$ & $0: 06: 22$ \\
\hline $15,000,000$ & $95.28 \%$ & $0: 07: 45$ \\
\hline $20,875,000$ & $95.73 \%$ & $0: 00: 07$ \\
\hline
\end{tabular}

Table 6.7 RDT optimization: achieved reliability depending on budget 
Table 6.7 also shows the required processor time of the LINGO $^{\mathrm{TM}}$ global solver to solve the NLP for $n=5$ components and $m_{P}=9$ available test procedures in the test procedure database. With the given complexity of the problem, i.e. the number of components and test procedures, the time required to solve the NLP is significantly influenced by the available budget for validation, ranging from approximately $10 \mathrm{~s}$ to more than $400 \mathrm{~s}$. The data on processor time is related to a computer system with 2010 hardware as described in Appendix 5.

For a significantly higher complexity of the system with more components and test procedures the exact solution of the NLP may not be possible within a suitable time. If so, dedicated procedures to find a reasonably good approximation have to be developed. Such procedures, which could for example be based on genetic algorithms, are not discussed within this research work.

Figure 6.2 illustrates the relationship between the validation effort and the LCL of system reliability as described above as well as the required processor time to solve the NLP with LINGO ${ }^{\mathrm{TM}}$. 


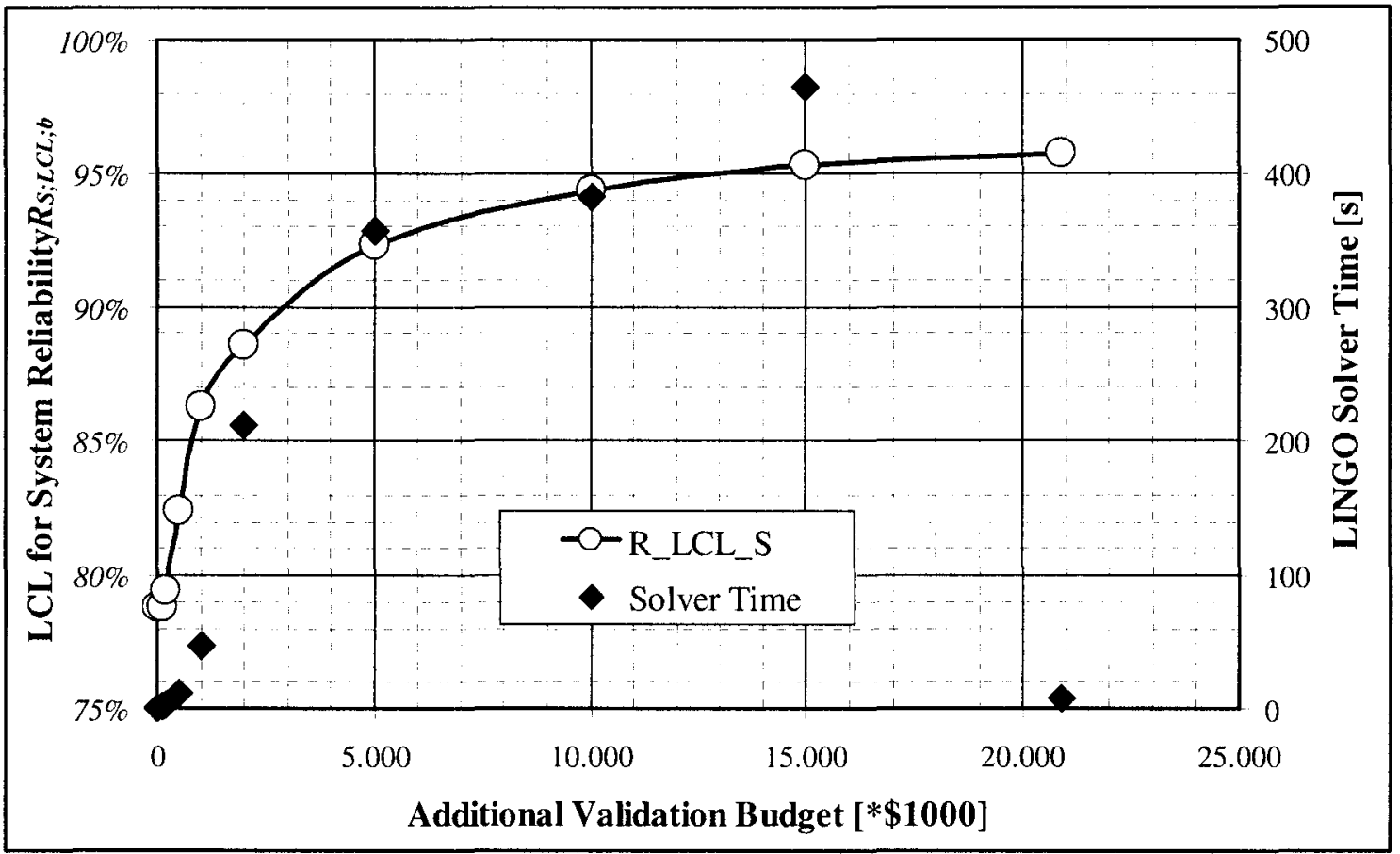

Figure 6.2 LCL of system reliability for different validation budgets

\subsection{Model for Optimizing RDT Plans with Respect to Cost}

In this section, a model is presented that can be used to minimize the validation $\operatorname{cost} C_{t}$ for achieving a certain required level of the LCL of system reliability. As discussed before, the variable $i(i=1 . . n)$ denotes a component. Tests that are already finished are indicated with the variable $j\left(j=l . . m_{F}\right)$ and the index $F$. Future tests, that are only planned so far, are denoted with the variable $k\left(k=1 . . m_{P}\right)$ and the index $P$. The result is an efficient RDT plan, similar to the last model.

The decision variables of the optimization problem are:

- duration of the planned test according to test type $k: t_{P k}$

- $\quad$ sample size for the test according to test type $k: l_{P k}$ 
The parameters of the NLP are:

- duration (i.e. reference for the statement on system reliability): $t_{0}$,

- confidence level: $b$

- component Weibull shape parameter of component $i: \beta_{i}$

- information about the finished reliability tests:

test durations $t_{F i j}$ that indicate the relevant test duration of test $j$ for component $i$ (remark: $t_{F i j}$ already considers acceleration factors $A F_{F i j}$ and the Boolean variable $o_{F i j}$ for indication if the test is obsolete or relevant for component $i$ )

- lower and upper bound for the test duration and the sample size according to test type $k: t_{P, \min k} / t_{P, \max k}$ and $l_{P, \min k} / l_{P, \max k}$

- calculatory speed to transform the duration of test $k$ from a hourly base into a vehicle mileage equivalent: $v_{P}$

- acceleration factor of test $k$ for component $i: A F_{P i k}$

- fixed cost of one test according to test type $k$ : $C_{f k}$

- variable cost of test type $k$ for one unit of test duration (time or mileage): $C_{v k}$

- required LCL of system reliability tests: $R_{S ; L C L ; b ; m i n}$ 
Thus the resulting NLP to determine the RDT plan with minimal validation cost is:

\section{Minimize $C_{t}$}

subject to

a) $R_{S ; L C L ; b}\left(t_{0}\right)=\operatorname{Min}_{i}\left[b^{\left(\frac{t_{0}^{\beta_{i}}}{T W_{i}^{\beta_{i}}}\right)}\right] \geq R_{S ; L C L ; b ; \min }$

b) $T W_{i}^{\beta_{i}}=\sum_{j=l}^{m_{F}} t_{F i j} \beta_{i}$

$$
+\sum_{k=1}^{m_{P}}\left[l_{P k} *\left(t_{P k} * v_{P k} * A F_{P i k}\right)^{\beta_{i}}\right] \quad \text { for } i=1, \ldots, n
$$

c) $C_{t k}=l_{P k} *\left(C_{f k}+C_{v k} * t_{P k}\right)$ for $k=1, \ldots, m_{P}$

d) $\sum_{k=1}^{m_{P}} C_{t k}=C_{t}$

e) $t_{P, \min k} \leq t_{P k} \leq t_{P, \max k} \quad$ for $k=1, \ldots, m_{P}$

f) $l_{P, \min k} \leq l_{P k} \leq l_{P, \max k} \quad$ for $k=1, . ., m_{P}$

g) $l_{P k}=[0 ; 1 ; 2 ; 3 ; \ldots .$.$] \quad for k=1, . ., m_{P}$

h) $t_{0} ; A F_{P i k} ; v_{P k} ; \beta_{i} \geq 0 \quad$ for $i=1, . ., n ;$ for $k=1, . ., m_{P}$

i) $0 \leq b \leq 1$ 
Where:

a) calculation of the $R_{S ; L C L ; b}$ according to the first theorem (3.15). It has to be higher than the required $R_{S ; L C L ; b, \min }$.

b) calculation of the Weibull equivalent single test duration $T W_{i}$ according to (3.5) for all components $i$ considering completed and future tests

c) calculation of the total cost (variable and fixed costs) for all tests $k$

d) calculation of the objective, the grand total cost of all tests

e) all tests $k$ meet the constraints on minimum and maximum duration

f) all tests $k$ meet the constraints on minimum and maximum sample size

g) the sample size of all tests $k$ are integer

h) the reference time $t_{0}$, each acceleration factor $A F_{i j}$, the calculatory speed $v_{P k}$ of each test $k$ and all Weibull parameters $\beta_{i}$ must not be negative

i) the confidence level $b$ must be between $0 \%$ and $100 \%$

All the solutions of the NLP (6.1) of section 6.3 for a certain validation budget are optimal RDT plans since they all lead to the maximum LCL of system reliability within the existing constraints. This also applies for the solution of NLP (6.2) that minimizes cost. The respective points for each solution of the NLP lead to the curve that limits the marked area in the upper diagram of Figure 6.1.

\subsection{Model for Optimizing RDT Plans with Respect to LCL of $B_{X}$ - lifetime}

Comparable to the approaches above it is also possible to formulate a NLP for the optimization of RDT plans with respect to the LCL of $B_{X}$ - lifetime. Like before the variable $i(i=1 . . n)$ denotes a component. Tests that are already finished are indicated with the variable $j\left(j=1 . . m_{F}\right)$ and the index $F$. Future tests, that are only planned so far, are denoted with the variable $k\left(k=1 . . m_{P}\right)$ and the index $P$. 
The decision variables of the optimization problem are:

- duration of the planned test according to test type $k: t_{P k}$

- $\quad$ sample size for the test according to test type $k: l_{P k}$

The parameters of the NLP are:

- share of systems that are allowed to fail before duration $B_{X}: X$

- confidence level: $b$

- component Weibull shape parameter of component $i: \beta_{i}$

- information about the finished reliability tests:

test durations $t_{F i j}$ that indicate the relevant test time of test $j$ for component $i$ (remark: $t_{F i j}$ already considers acceleration factors $A F_{F i j}$ and the Boolean variable $o_{F i j}$ for indication if the test is obsolete or relevant for component $i$ )

- lower and upper limit for the test duration according to test type $k$ : $t_{P, \min k}$ and $t_{P, \max k}$

- lower and upper limit for the sample size according to test type $k$ : $l_{P, \min k}$ and $l_{P, \max k}$

- calculatory speed to transform the duration of test $k$ from a hourly base into a vehicle mileage equivalent: $v_{P k}$

- acceleration factor of test $k$ for component $i: A F_{P i k}$

- fixed cost of one test according to test type $k: C_{f k}$

- variable cost of test type $k$ for one unit of test duration (time or mileage): $C_{v k}$

- maximum validation budget for planned all tests: $C_{t \max }$

The following NLP (6.3) allows determining the RDT plan with the highest LCL of $B_{X}$ lifetime within the given constraints including total validation budget. 


$$
\begin{aligned}
& \text { Maximize } B_{X ; S ; L C L ; b} \\
& \text { subject to } \\
& \text { a) } B_{X ; S ; L C L ; b}=\operatorname{Min}_{i}\left[\left(\frac{\ln \left(1-\frac{x}{100 \%}\right) * T W_{i}^{\beta_{i}}}{\ln (b)}\right)^{l / \beta_{i}}\right] \\
& \text { b) } T W_{i}^{\beta_{i}}=\sum_{j=1}^{m_{F}} t_{F i j} \beta_{i} \\
& +\sum_{k=1}^{m_{P}}\left[l_{P k} *\left(t_{P k} * v_{P k} * A F_{P i k}\right) \beta_{i}\right] \quad \text { for } i=1, . ., n \\
& \text { c) } C_{t k}=l_{P k} *\left(C_{f k}+C_{v k} * t_{P k}\right) \text { for } k=1, \ldots, m_{P} \\
& \text { d) } \sum_{k=1}^{m_{P}} C_{t k} \leq C_{t \max } \\
& \text { e) } t_{P, \min k} \leq t_{P k} \leq t_{P, \max k} \quad \text { for } k=1, \ldots, m_{P} \\
& \text { f) } l_{P, \min k} \leq l_{P k} \leq l_{P, \max k} \quad \text { for } k=1, \ldots, m_{P} \\
& \text { g) } l_{P k}=[0 ; 1 ; 2 ; 3 ; \ldots . .] \quad \text { for } k=1, . ., m_{P} \\
& \text { h) } t_{0} ; A F_{P i k} ; v_{P k} ; \beta_{i} \geq 0 \quad \text { for } i=1, . ., n ; \text { for } k=1, . ., m_{P} \\
& \text { i) } 0 \leq b \leq 1
\end{aligned}
$$

For an explanation of the constraints see NLP (6.1). The NLP is very similar with the exception that NLP (6.3) optimizes with respect to LCL of $B_{X}$ - lifetime. 
Likewise as for the LCL of system reliability it is also possible to invert the optimization to find the minimum validation cost for a given target on LCL of system $B_{X}$ - lifetime. The respective NLP is given in (6.4).

Minimize $C_{t}$

subject to

a) $B_{X ; S ; L C L ; b}=\operatorname{Min}_{i}\left[\left(\frac{\ln \left(1-\frac{x}{100 \%}\right) * T W_{i}^{\beta_{i}}}{\ln (b)}\right)^{1 / \beta_{i}}\right] \geq B_{X ; S ; L C L ; b ; \operatorname{Min}}$

b) $T W_{i}^{\beta_{i}}=\sum_{j=l}^{m_{F}} t_{F i j} \beta_{i}$

$+\sum_{k=1}^{m_{P}}\left[l_{P k} *\left(t_{P k} * v_{P k} * A F_{P i k}\right) \beta_{i}\right]$ for $i=1, \ldots, n$

c) $C_{t k}=l_{P k} *\left(C_{f k}+C_{v k} * t_{P k}\right) \quad$ for $k=1, . ., m_{P}$

d) $\sum_{k=1}^{m_{P}} C_{t k}=C_{t}$

e) $t_{P, \min k} \leq t_{P k} \leq t_{P, \max k} \quad$ for $k=1, . ., m_{P}$

f) $l_{P, \min k} \leq l_{P k} \leq l_{P, \max k} \quad$ for $k=1, \ldots, m_{P}$

g) $l_{P k}=[0 ; 1 ; 2 ; 3 ; \ldots .$.$] \quad for k=1, . ., m_{P}$

h) $t_{0} ; A F_{P i k} ; v_{P k} ; \beta_{i} \geq 0 \quad$ for $i=1, . ., n$; for $k=1, . ., m_{P}$

i) $0 \leq b \leq 1$ 
For an explanation of the constraints see NLP (6.2). The NLP is very similar with the exception that constraint a) in NLP (6.4) is on LCL of $B_{X}$ - lifetime instead of system reliability.

\subsection{Disadvantages of Identical Test Duration for all Units}

In all situations in which components suffer wear, aging or other forms of degradations, an increasing failure rate can be observed. This is represented with a Weibull parameter $\beta$ greater than 1 . For these components longer test durations contribute disproportionately to the reliability proof according to the definition of the Weibull equivalent single test duration $T W_{i}$ in equation (3.4).

Since the number of tests per definition can only be integer, the following situation can occur while optimizing the RDT program:

If the test duration in the optimal solution according to one of the NLPs (6.1) to (6.4) was not at the maximum test duration and the sample size is greater than 1 , there might be an even better solution if all units except one are tested to the maximum possible duration. The duration of the remaining unit then will be used to fine-tune the respective reliability to fulfill the constraints like minimum required reliability.

This will be illustrated in an example. The starting point is the example introduced above in section 6.4. The information about completed tests and the resulting LCL of system reliability is shown in Table 6.8 . 


\begin{tabular}{|c|c|c|c|c|c|c|}
\hline & & 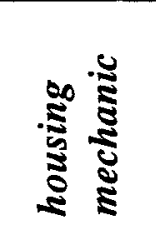 & 焉 & 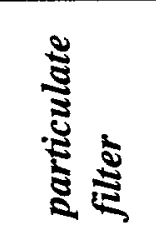 & 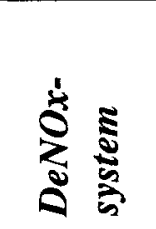 & 芯 \\
\hline Weibull Parameter & $\bar{\beta}$ & 1.5 & 2.0 & 1.8 & 1.3 & 1.0 \\
\hline Duration to & kmiles & 400 & & & & \\
\hline Conf. Level $b$ & - & 0.10 & & & & \\
\hline TWi finished tests & kmiles & 2170.6 & 2488.3 & 1410.2 & 2965.4 & 5071.0 \\
\hline$R \_L C L \_C i$ & - & $83.35 \%$ & $94.22 \%$ & $78.79 \%$ & $84.34 \%$ & $83.39 \%$ \\
\hline$R \_L C L \_S$ & - & $78.79 \%$ & & & & \\
\hline
\end{tabular}

Table 6.8 Demonstrated LCL of reliability prior to additional RDT

Based on the completed durability tests, a LCL of system reliability of $78.79 \%$ was proven with the confidence level $b=0.10$. This system reliability demonstration is not considered to be high enough. Therefore additional validation testing should be conducted. For simplification in this example there is just one test procedure available, the so called "express-run". Table 6.9 shows the ALT acceleration factors that can be achieved with this test procedure in comparison to the duty cycle of the reference customer application.

\begin{tabular}{|c|c|c|c|c|c|c|c|}
\hline 㫋 & 离 & 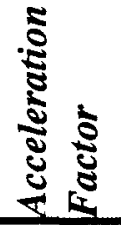 & 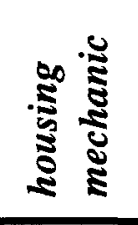 & 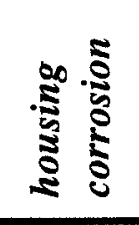 & 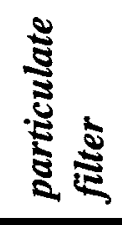 & 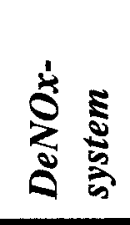 & 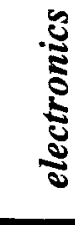 \\
\hline$C$ & express-run & $A F$ & 1 & 1 & 1.5 & 1.5 & 1 \\
\hline
\end{tabular}

Table 6.9 ALT acceleration factors for express-run

The constraints for RDT planning on sample size and duration of the durability tests as well as the cost associated with the test procedure "express-run" are listed in the Table 6.10: 


\begin{tabular}{|c|c|c|}
\hline & $\min$ & $\max$ \\
\hline duration & 30 & 300 \\
\hline sample size & 0 & 10 \\
\hline fixed costs (per test) & \multicolumn{2}{|c|}{$\$ 150,000$} \\
\hline variable costs (per kmile) & \multicolumn{2}{|c|}{$\$ 1,250$} \\
\hline
\end{tabular}

Table 6.10

Constraints and Cost for Express-Run

The direct application of the NLPs according to (6.1) or (6.2) would lead to the relationship between additional validation budget and LCL of system reliability as illustrated in Figure 6.3. Each curve in this figure represents a different number of units on test and shows the test cost versus the resulting reliability contribution for different test durations between the minimum and maximum constraints as defined above.

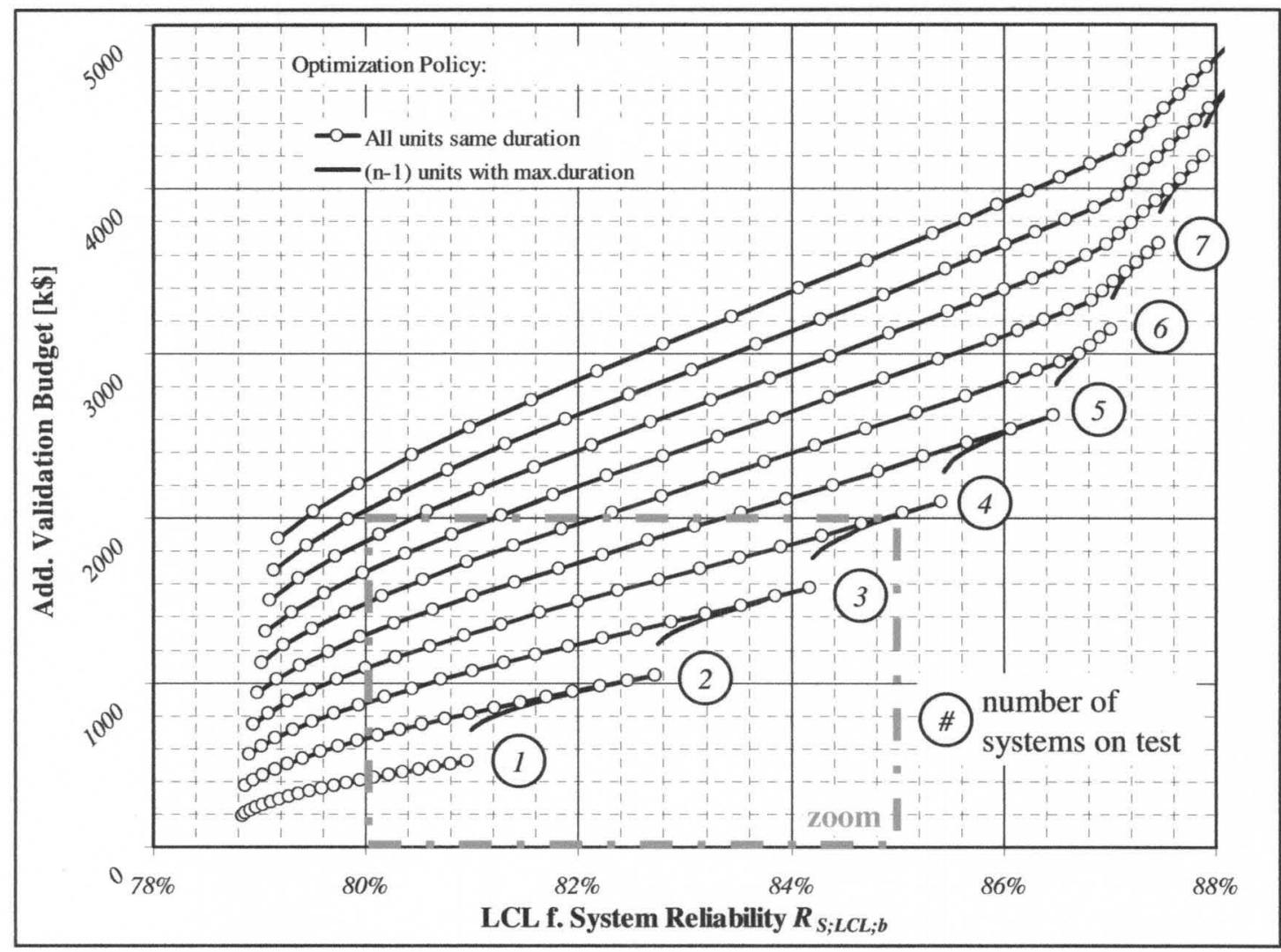

Figure 6.3 Relationship between LCL of system reliability and validation cost 
There is a large increase in cost at the end of each line that represents the maximum test duration. If the respective reliability is not sufficient to meet the constraints, an additional unit will have to be put on test. An improved policy for such situations is recommended to account for the disproportionate influence of test duration for $\beta>1$ :

- All units except one are put on the to the maximum possible test duration.

- The duration of the remaining unit is used to fine-tune the LCL of system reliability.

Figure 6.3 illustrates the curves for this policy for test optimization. This is illustrated even more clearly in the next Figure 6.4 that zooms into the diagram of Figure 6.3. The areas where a modified optimization policy increases the efficiency of the RDT program are shaded.

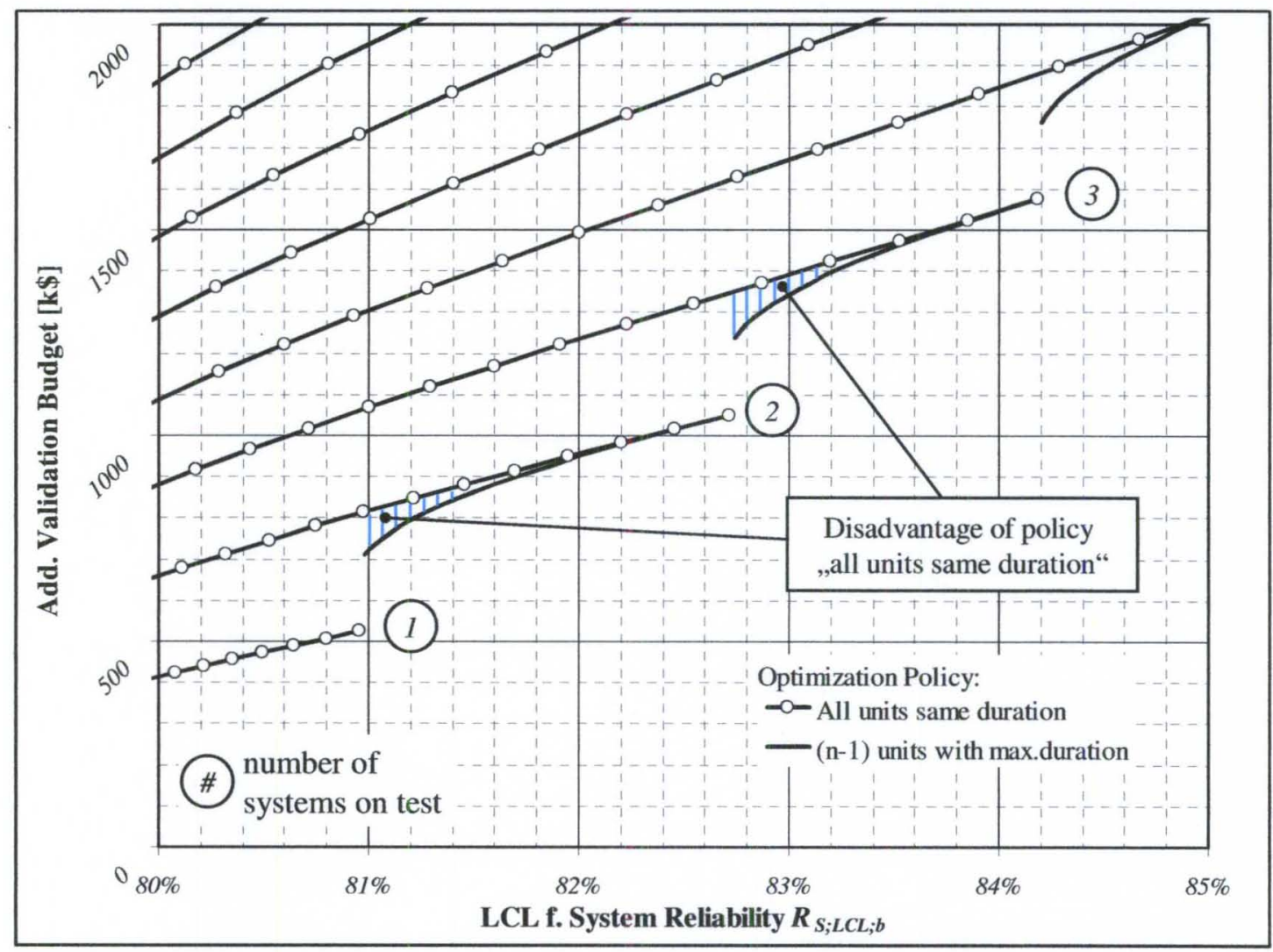

Figure 6.4 Advantage of modified optimization policy 
The modifications to optimize according to the proposed policy are shown in NLP (6.5). This NLP is related to an optimization with respect to the LCL of system reliability and is based on NLP (6.1).

A similar modification of the NLP to consider the improved optimization policy could also take place in three other situations:

- optimization with respect to cost to achieve the required system reliability

- optimization with respect to $B_{X}$ - lifetime within validation budget constraints

- optimization with respect to cost for a required $B_{X}$ - lifetime

The decision variables of the modified optimization problem considering the improved policy are:

- duration of the planned tests according to test type $k: t_{P k}$

- $\quad$ sample size for the test according to test type $k: l_{P k}$

- duration of one additional planned test according to test type $k: t_{P *}$

- boolean variable to indicate if there is one additional planned test [ yes / no ] according to test type $k$ that has a modified duration: $l_{P * k}$

The parameters of the NLP are:

- duration (i.e. reference for the statement on system reliability): $t_{0}$

- confidence level: $b$

- component Weibull shape parameter of component $i: \beta_{i}$

- information about the finished reliability tests: test durations $t_{F i j}$ that indicate the relevant test time of test $j$ for component $i$ (remark: $t_{F i j}$ already considers acceleration factors $A F_{F i j}$ and the Boolean variable $o_{F i j}$ for indication if the test is obsolete or relevant for component $i$ )

- lower and upper limit for the test duration according to test type $k$ : $t_{P, \min k}$ and $t_{P, \max k}$

- lower and upper limit for the sample size according to test type $k$ : $l_{P, \min k}$ and $l_{P, \max k}$ 
- calculatory speed to transform the duration of test $k$ from a hourly base into a vehicle mileage equivalent: $v_{P k}$

- $\quad$ acceleration factor of test $k$ for component $i: A F_{P_{i k}}$

- fixed cost of one test according to test type $k$ : $C_{f k}$

- variable cost of test type $k$ for one unit of test duration (time or mileage): $C_{v k}$

- maximum validation budget for planned all tests: $C_{t \max }$

Where:

a) calculation of the objective, $R_{S ; L C L ; b}$, according to the first theorem (3.15).

b) calculation of the Weibull equivalent single test duration $T W_{i}$ according to (3.5) for all components $i$ considering completed and future tests

c) calculation of the total cost (variable and fixed costs) for all tests $k$

d) constraint that the grand total cost of all test costs is within the budget

e) all tests $k$ meet the constraints on minimum and maximum duration

f) all tests $k$ meet the constraints on minimum and maximum sample size

g) the sample size of all tests $k$ are integer; there is either a single test of type $k$ with different duration or not

h) the reference time $t_{0}$, each acceleration factor $A F_{i j}$, the calculatory speed $v_{P k}$ of each test $k$ and all Weibull parameters $\beta_{i}$ must not be negative

i) the confidence level $b$ must be between $0 \%$ and $100 \%$

The NLP to determine the RDT plan with optimal system reliability according to the improved optimization policy is: 
Maximize $R_{S ; L C L ; b}$

subject to

a) $R_{S ; L C L ; b}\left(t_{0}\right)=\operatorname{Min}_{i}\left[b^{\left(\frac{t_{0}^{\beta_{i}}}{T W_{i}^{\beta_{i}}}\right)}\right]$

b) $T W_{i}^{\beta_{i}}=\sum_{j=1}^{m_{F}} t_{F i j} \beta_{i}+\sum_{k=1}^{m_{P}}\left[l_{P k} *\left(t_{P k} * v_{P k} * A F_{P i k}\right) \beta_{i}\right]$ $+\sum_{k=1}^{m_{P}}\left(l_{P^{*} k} * t_{P^{*} k} * v_{P k} * A F_{P i k}\right)^{\beta_{i}} \quad$ for $i=1, \ldots, n$

c) $C_{t k}=\left(l_{P * k}+l_{P k}\right) *\left(C_{f k}+C_{v k} * t_{P k}\right) \quad$ for $k=1, \ldots, m_{P}$

d) $\sum_{k=1}^{m_{P}} C_{t k} \leq C_{t \max }$

e) $t_{P, \min k} \leq t_{P k} \leq t_{P, \max k} \quad$ for $k=1, . ., m_{P}$

f) $l_{P, \min k} \leq l_{P^{*} k}+l_{P k} \leq l_{P, \max k} \quad$ for $k=1, \ldots, m_{P}$

g) $l_{P k}=[0 ; 1 ; 2 ; 3 ; \ldots ..] ; l_{P^{*} k}=[0 ; 1] \quad$ for $k=1, . ., m_{P}$

h) $t_{0} ; A F_{P i k} ; v_{P k} ; \beta_{i} \geq 0 \quad$ for $i=1, \ldots, n ;$ for $k=1, . ., m_{P}$

i) $0 \leq b \leq 1$ 
As mentioned above the NLPs (6.2) to (6.4) for optimization with respect to other variables than LCL of system reliability could be modified in a similar way as in (6.1) to (6.5) with the introduction of $l_{P^{*} k}$ and $t_{P^{*} k}$. An optimization model with respect to validation cost based on (6.2) will be presented in Chapter 7.5 in an even more extended way that additionally considers the influence of customer usage in different segments.

Unfortunately the drawback associated with this improved policy for RDT optimization is the additional complexity of the mixed-integer non-linear optimization problem. This can increase the solution time significantly. The reason for this is the fact that in parallel to each integer variable $l_{P k}$ an additional Boolean variable $l_{P^{*} k}$ now has to be considered. Thus for large size RDT optimization problems with a large number of components and possible test procedures it is not recommended to apply this policy for the sake of processor time of the solver software (for example LINGO $^{\mathrm{TM}}$ ).

All NLP (6.1) through (6.5) could also be combined with the NLP (5.1) from section 5.2 to consider the $\beta$ sensitivity. But this is not recommended since there is a very high additional complexity and therefore a significant increase in processing time. A compromise could be an approach that optimizes the RDT program first with fixed $\beta_{i}$ assumed. Afterwards based on the optimization result a $\beta$ sensitivity study is conducted to analyze the robustness of the solution concerning variations in $\beta$. Depending on the outcome of the $\beta$ sensitivity study a second optimization run follows with adapted values for the fixed $\beta_{i}$. 


\subsection{Integration of RDT Optimization into the Reliability Growth Process}

As presented in Chapter 2.3, Reliability Growth is an iterative process for the validation of a product that starts after finishing the design. Prototypes are built and tested in an RDT program until failures occur. The root causes of these failures are identified and adequate countermeasures are implemented, either in the design or in subsequent processes like manufacturing or assembly. Updated parts are tested again in the RDT program until they fail again. The iterative process of testing, failing, analyzing and correcting goes on until the product has achieved its targeted reliability. This is illustrated in the left part of Figure 6.5.

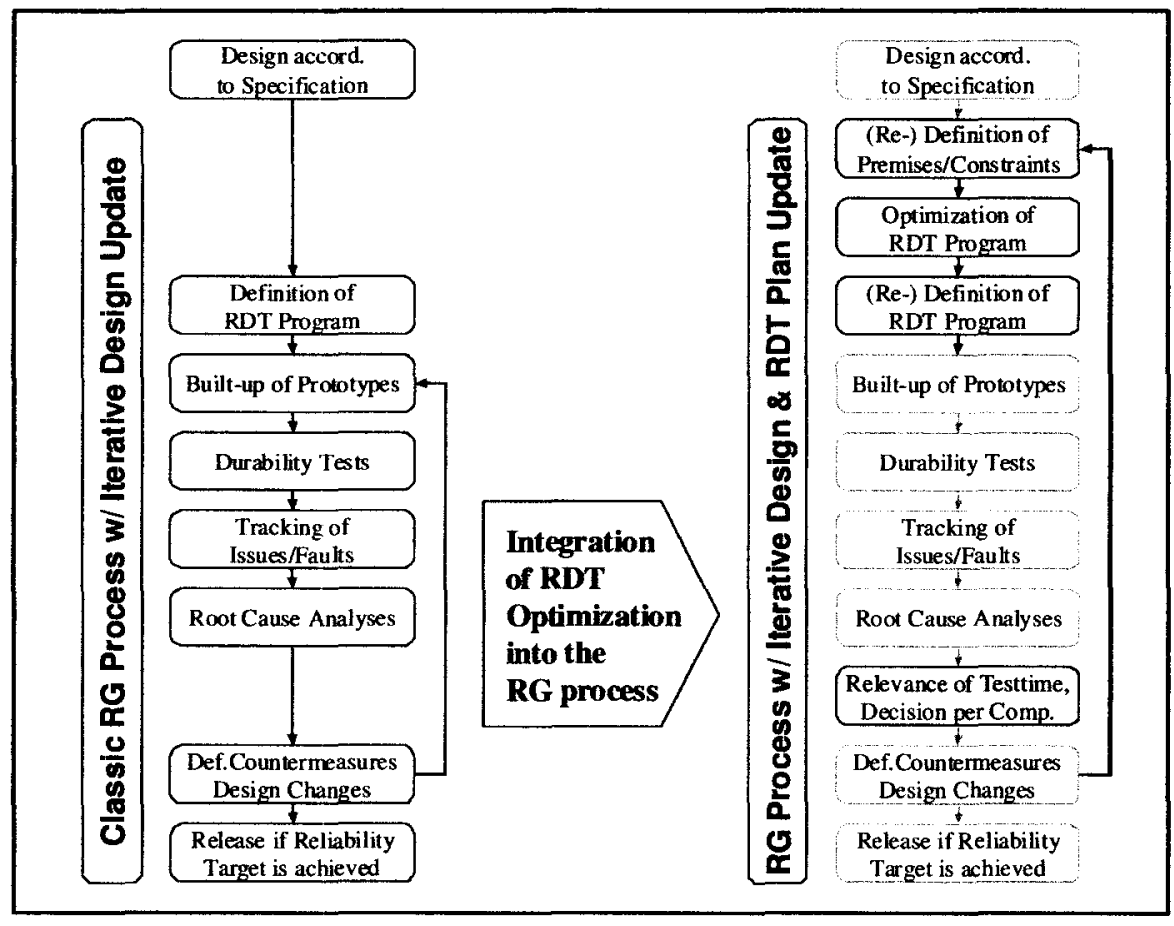

Figure 6.5 Integration of RDT Optimization into the Reliability Growth Process 
The right hand side of Figure 7.1 shows a new approach that includes the optimization of the RDT Program using the NLPs introduced in Chapter 6. Building prototypes and testing within the RDT program is comparable to the classic approach with the exception that the RDT program is optimized prior to the start of the durability runs to make sure that it is efficient. Such an "optimization" is often made intuitively by the quality engineer and not as an optimization in a mathematical sense. The additional process steps are shaded in Figure 6.5.

During testing the occurrence of failures cannot be avoided and their root causes have to be analyzed and adequate countermeasures have to be taken. As described in the RDT framework (see 3.2), after each issue during RDT and each implementation of a design change, there must be a decision per test and per component as to whether or not the accumulated test time should be treated as relevant or obsolete. Based on these decisions and potential updates in the constraints or targets of the validation the optimization of the RDT plan is updated. This is a new process step that can lead to a re-allocation of resources. But this step ensures that the further RDT program that will be conducted from this point on is still optimal also under the current boundary conditions. 


\section{CONSIDERING DIFFERENT CUSTOMER SEGMENTS AND THE RESPECTIVE LOAD FACTORS}

In the previous chapters of the dissertation there was a deterministic view on the loads or stresses on the system and its components. These loads or stresses were considered to be constant. The only variation in the lifetime of the technical system was assumed to be caused by the distribution in lifetime of the components. In reality the lifetime of systems can also be influenced by customer usage and the component stress level caused by the load cycle in the respective customer segment. Using such information allows for more accurate predictions on system and component reliability and lifetime compared to an approach that does not differentiate between the customer segments.

\subsection{Calculation of Cumulative Reliability Across Different Segments with Total Reliability}

In this section, a methodology for consideration of information about different customer segments and their usage will be presented. The share of each customer segment $l$ in relation to the total production volume is denoted as $S_{l}$.

Figure 7.1 gives a simple numerical example to show how information about the share of customer segments and the LCL of system reliability within the segments can be utilized to make statements on the system reliability across all customer segments. 


\section{Consideration of Different Customer Segments}

\section{LCL for System Reliability}

- Segment A $\left(S_{A}=75 \%\right)$

$$
R_{S ; S M A ; L C L ; b}=0.95
$$

- Segment B $\left(S_{B}=25 \%\right) \quad\left(S_{A}+S_{B}=100 \%\right)$

$R_{S ; S M B ; L C L ; b}=0.75$

- In Segment A not more than $0.75(1-0.95) * 100 \%=3.75 \%$ of all produced systems fail (LCL).

- In Segment B not more than $0.25 *(1-0.75) * 100 \%=6.25 \%$ of all produced systems fail (LCL).

- In total not more than $10 \%$ of all systems fail (LCL):

$$
\begin{aligned}
& R_{S ; L C L ; b}=0.90=1-S_{A} *\left(1-R_{S ; S M A ; L C L ; b}\right)-S_{B} *\left(1-R_{S ; S M B ; L C L ; b}\right) \\
& R_{S ; L C L ; b}=S_{A} R_{S ; S M A ; L C L ; b}+S_{B} R_{S ; S M B ; L C L ; b}
\end{aligned}
$$

Figure 7.1 Example for the consideration of reliability of customer segments

The simple example in Figure 7.1 shows that the cumulative reliability $R_{A S}$ of an item, which is utilized in $l_{\max }$ different customer segments, can be calculated with the reliability $R_{S M l}$ in each segment $l$ and the respective share of the segment $S_{l}$. The sum of the shares of all customer segments is equal to 1 . The cumulative reliability $R_{A S}$ is 1 minus the percentage of systems failing in the field over all customer segments.

$$
\begin{aligned}
& R_{A S}=1-\sum_{l=1}^{l_{\max }}\left(S_{l} *\left(1-R_{S M l}\right)\right)=\sum_{l=1}^{l_{\max }}\left(S_{l} * R_{S M l}\right) \\
& \sum_{l=1}^{l_{\max }} S_{l}=1
\end{aligned}
$$




\section{Cumulative reliability across different segments}

The cumulative reliability across different customer segments is the sum of reliabilities in the segments weighted with the share of the segments. This can be applied to reliability as well as to the LCL of reliability. The cumulative LCL of the reliability is 1 minus the expected overall percentage of failed system of the total production volume.

In equation (7.1) the share $S_{l}$ can be understood as the reliability of certain specific items being utilized in the segment $l$. In this case the reliability $R_{S M l}$ is the conditional reliability of the item given that it is utilized in segment $l$. So equation (7.1) is basically an application of the rule of total probability ${ }^{20}$, as illustrated in equation (7.3) where $P(B)$ is the cumulative reliability and the event $P(\bar{B})$ is a failure in the field. The events $E_{1}, E_{2}, E_{3}, \ldots$ represent the belonging to the respective customer segment $1,2,3, \ldots$. The rule of total probability is given as:

$P(B)=P\left(B \cap E_{1}\right) * P\left(E_{1}\right)+P\left(B \cap E_{2}\right) * P\left(E_{2}\right)+P\left(B \cap E_{3}\right) * P\left(E_{3}\right)+\ldots$

with: $P\left(E_{1}\right)+P\left(E_{2}\right)+P\left(E_{3}\right)+\ldots=1$

The equation (7.1) can not only be applied to probabilities like reliabilities but also to the LCLs of the respective reliability. So the LCL of cumulative reliability across all customer segments $R_{A S ; L C L ; b}$ is:

$$
R_{A S ; L C L ; b}=\sum_{l=1}^{l_{\max }}\left(S_{l} * R_{S M l ; L C L ; b}\right)
$$

\footnotetext{
${ }^{20}$ see Montgomery and Runger (2003) p.44
} 


\subsection{Calculating Reliability Considering Component Specific Stress Factors}

As described above the customer usage can have a significant influence on component and system reliability. Let $d_{A l}$ denote the annual mileage and $t_{0}$ the reference time, for example the duration of the warranty period. The customer segment is indicated with the variable $l$. The stress factors $S F_{i l}$ for each component $i$ are representing the speed of cumulative damage per mile in segment $l$ in comparison to the reference usage profile.

The stress factors $S F_{i l}$ are related to the ALT acceleration factors $A F_{i j}$ introduced in 3.3 for the definition of the Weibull equivalent single test durations durationTW $W_{i}$ But $S F_{i l}$ is utilized to model the effects on reliability resulting from customer usage whereas $A F_{i j}$ represents the acceleration resulting from a certain test procedure.

The reliability of component $i$ in customer segment $l$ follows the Weibull law according to equation (2.10). The reliability after the time $t_{0}$ is:

$R_{i l}\left(t_{O}\right)=e^{-\left(\frac{t_{0}{ }^{*} d_{A l} * S F_{i l}}{\theta_{i}}\right)^{\beta_{i}}}$

With the definitions above and equation (3.14) $R_{C i ; S M ~ l ; L C L ; b}$, the LCL of reliability of component $i$ after time $t_{0}$ in customer segment $l$, yields to:

$R_{C i ; S M l ; L C L ; b}\left(t_{0}\right)=b^{\left(\frac{\left(t_{0} * d_{A l} * S F_{i l}\right)^{\beta_{i}}}{T W_{i}^{\beta_{i}}}\right)}$

The respective LCL of system reliability in this customer segment $l$ can be calculated by substituting (7.7) into equation (3.15): 


$$
R_{S ; S M ~ l ; L C L ; b}\left(t_{0}\right)=\operatorname{Min}_{i}\left[b^{\left(\frac{\left(t_{0} * d_{A l}{ }^{*} S F_{i l}\right.}{T W_{i}^{\beta_{i}}}\right)}\right]
$$

\subsection{Model for Cumulative Reliability Across Different Customer Segments}

Combining the two equations (7.5) for the consideration of customer segments and (7.7) for the influence of stress factors the cumulative LCL of reliability of component $i$ over all customer segments $l$ (with $l=1 . . l_{\max }$ ) is $R_{C i ; A S ; L C L ; b}$ :

$$
R_{C i ; A S ; L C L ; b}\left(t_{0}\right)=\sum_{l=1}^{l_{\max }}\left(S_{l} * b\left(\frac{\left(t_{0} * d_{A l} * S F_{i l}\right)^{\beta_{i}}}{T W_{i}^{\beta_{i}}}\right)\right)
$$

The LCL of cumulative reliability across different segments, as it was defined in section 7.1 , is the LCL of reliability (of the system or of a specific component) across all customer segments. 
By using equation (3.15) the LCL of cumulative system reliability $R_{S ; A S ; L C L ; b}$ over all segments is correspondingly:

$$
R_{S ; A S ; L C L ; b}\left(t_{0}\right)=\sum_{l=1}^{l_{\max }}\left(S_{l}^{*} \operatorname{Min}_{i}\left[b^{\left(\frac{\left(t_{0} * d_{A l} * S F_{i l}\right)^{\beta_{i}}}{T W_{i}^{\beta_{i}}}\right)}\right]\right)
$$

Notation for (7.9) and (7.10):

- customer segments: $l\left(l=1 . . l_{\max }\right)$

- share of the customer segment $l$ in relation to the total production volume: $S_{l}$

- $\quad$ stress factor of the customer usage in segment $l$ for component $i: S F_{i l}$

- annual mileage in customer segment $l: d_{A} l$

- reference time, for example warranty period: $t_{0}$

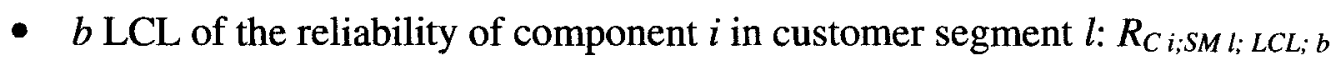

- $\quad b$ LCL of the system reliability in customer segment $l: R_{S ; S M} l ; L C L ; b$

- annual mileage in customer segment $l: d_{A} l$

\subsection{Example for Consideration of Customer Usage Specific Stress Factors}

This example is also based on the context of the previously introduced example for RDT of an exhaust gas aftertreatment system. Table 7.1 includes the durations of the completed test multiplied by the component specific acceleration factors. For tests with damages that have caused the implementation of countermeasures, the test duration for the respective components was set to 0 . 
The system contains the following well known components:

- housing with focus on mechanical failures

- housing with focus on corrosion

- particulate filter system

- DeNOx-System including the SCR-catalsyst

- electronics

The reliability statement is related to an operation time of $t_{0}=3$ years which for example could represent the warranty period. With an annual mileage of $130 \mathrm{kmiles}$ the total mileage within the period $t_{0}=3$ years is 390 kmiles.

The $b=0.1 \mathrm{LCL}$ of system reliability at time $t_{0}=3$ years is $R_{S ; L C L ; b}=79.43 \%$ with the particulate filter being the limiting component. The LCLs of reliability of the other components for time $t_{0}$ are also given in Table 7.1. These LCLs of the reliabilities of the system and components are computed according to equations (3.14) and (3.15). 
Accumulated Test Time (component specific ALT-factors are considered)

\begin{tabular}{|c|c|c|c|c|c|c|c|c|}
\hline 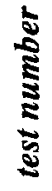 & 总 & $\stackrel{\vec{\Xi}}{\mathbf{S}}$ & 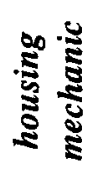 & 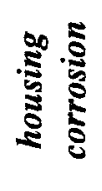 & 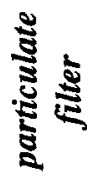 & 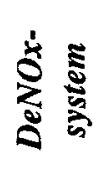 & $\frac{\mathfrak{s}}{\tilde{s}}$ & $\frac{5}{5}$ \\
\hline & & & \multicolumn{6}{|c|}{ effective mileage (per component) } \\
\hline 1 & hydropuls test & kmiles & 0 & 0 & 0 & 0 & 0 & - \\
\hline 2 & spray test & kmiles & 0 & 2250 & 0 & 750 & 750 & - \\
\hline 3 & hydropuls test & kmiles & 750 & 0 & 0 & 0 & 0 & - \\
\hline 4 & hydropuls test & kmiles & 750 & 0 & 0 & 0 & 0 & - \\
\hline 5 & spray test & kmiles & 750 & 225 & 0 & 750 & 750 & - \\
\hline 6 & customer testing & kmiles & 200 & 200 & 0 & 200 & 200 & - \\
\hline 7 & alternating load & kmiles & 0 & 0 & 600 & 0 & 600 & - \\
\hline 8 & spray test & kmiles & 750 & 225 & 750 & 750 & 750 & - \\
\hline 9 & WHTC cycle & kmiles & 0 & 150 & 0 & 450 & 300 & - \\
\hline 10 & corrosion test & kmiles & 0 & 750 & 0 & 0 & 0 & - \\
\hline 11 & city cycle & kmiles & 100 & 300 & 300 & 300 & 200 & - \\
\hline 12 & rough road test & kmiles & 0 & 21 & 21 & 21 & 21 & - \\
\hline 13 & city cycle & kmiles & 100 & 300 & 300 & 300 & 200 & - \\
\hline 14 & express-run & kmiles & 200 & 200 & 300 & 300 & 200 & - \\
\hline 15 & WHTC cycle & kmiles & 0 & 150 & 450 & 450 & 300 & - \\
\hline 16 & express-run & kmiles & 200 & 200 & 300 & 300 & 200 & - \\
\hline 17 & customer testing & kmiles & 200 & 200 & 200 & 200 & 200 & - \\
\hline 18 & customer testing & kmiles & 200 & 200 & 200 & 200 & 200 & - \\
\hline 19 & customer testing & kmiles & 200 & 200 & 200 & 200 & 200 & - \\
\hline
\end{tabular}

\section{System Information}

\begin{tabular}{|c|c|c|c|c|c|c|c|}
\hline Weibull Parameter & $\beta$ & 1.5 & 2.0 & 1.8 & 1.3 & 1.0 & - \\
\hline Annual Mileage & kmiles & 130 & in reference vehicle drive cycle & \\
\hline Years to & & 3 & Warranty Period \\
\hline Total Mileage & kmiles & 390 & within the Warranty Period \\
\hline Conf. Level b & - & 0.10 & \multicolumn{3}{l|}{} \\
\hline
\end{tabular}

LCL for System / Component Reliability (related to Reference Usage)

\begin{tabular}{|c|c|c|c|c|c|c|c|}
\hline$T W i$ & kmiles & 2170.6 & 2488.3 & 1410.2 & 2965.4 & 5071.0 & - \\
\hline$R \_L C L \_C i$ & - & $83.92 \%$ & $94.50 \%$ & $\mathbf{7 9 . 6 3 \%}$ & $84.81 \%$ & $83.77 \%$ & $\mathbf{7 9 . 6 3 \%}$ \\
\hline
\end{tabular}

Table 7.1 ALT results

In reality not all systems are operated in the same way as assumed in the reference cycle. In this example, there are 5 major customer segments to which this system is applied. Table 7.2 lists the customer segments including their share and annual mileages. The "long haul" segment has the overall highest share with $40 \%$ and was therefore chosen as reference cycle. 


\begin{tabular}{|c|c|c|}
\hline $\begin{array}{c}\text { Customer } \\
\text { Segment }\end{array}$ & $\begin{array}{c}\text { annual mileage } \\
\text { (kmiles) }\end{array}$ & Market Share \\
\hline Long Haul & 130 & $40 \%$ \\
\hline Pickup Heavy & 80 & $15 \%$ \\
\hline Urban & 60 & $10 \%$ \\
\hline Pickup Light & 40 & $15 \%$ \\
\hline Construction & 40 & $20 \%$ \\
\hline
\end{tabular}

Table 7.2 Customer segments, their annual mileage and market share

There is a big difference in how the trucks are typically operated in the different customer segments and therefore differences in the stress the respective components are exposed to. The differences in the drive cycle characteristics can be observed based on the following attributes:

- vehicle weight and loading: average and frequency distribution

- speed (engine/vehicle): average and frequency distribution

- engine torque (engine/vehicle): average and frequency distribution

- engine dynamic: number of accelerations per hour or per mile

- number of engine stops (per hour or per mile)

- percentage of idling time in relation to the total operation time

- exhaust gas temperature: average and frequency distribution

- ambient conditions like altitude, temperature, humidity

- contact with corrosive media

- mechanical load by road conditions

- application of engine brake: average and frequency distribution

The following Table 7.3 lists the effective stress factors for all components in every customer segment related to the vehicle miles. The stress factor of the long haul segment is 1.0 for all components. This means that the reference drive cycle of the system reflects exactly this customer segment which also has the highest market share. 


\begin{tabular}{|c|c|c|c|c|c|}
\hline $\begin{array}{c}\text { stress factors (mileage-based) } \\
\text { per component } \\
\text { per customer segment }\end{array}$ & 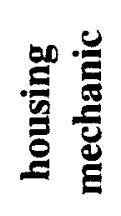 & 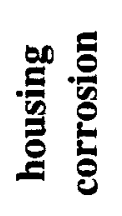 & 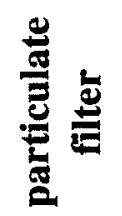 & 离宓 & $\frac{\mathscr{U}}{\stackrel{0}{0}}$ \\
\hline Long Haul & 1.0 & 1.0 & 1.0 & 1.0 & 1.0 \\
\hline Pickup Heavy & 1.0 & 1.5 & 1.0 & 1.0 & 1.0 \\
\hline Urban & 2.0 & 2.0 & 3.0 & 3.0 & 1.0 \\
\hline Pickup Light & 2.0 & 2.0 & 3.0 & 3.0 & 1.0 \\
\hline Construction & 4.0 & 3.0 & 2.0 & 3.0 & 1.0 \\
\hline
\end{tabular}

Table 7.3 Stress Factors for each component in all customer segments

Based on the given stress factors, the LCLs of the component reliability in each customer segment can be calculated according to equation (7.7). The lowest of these LCLs of component reliability for a certain customer segment is also the LCL of system reliability in the respective segment according to equation (3.15).

These values for the LCL of system reliability in each customer segment are given in the rightmost column of Table 7.4. For each segment the limiting component for the system reliability is marked with a shading of the respective matrix element. For example for the segment "construction" the LCL of system reliability for the warranty period of $t_{0}=3$ years is $78.71 \%$ with the mechanic part of the housing being limiting. This is related to the strong mechanical stress in construction vehicle resulting from the severe road conditions. Whereas for the "urban" segment, the LCL of system reliability is only $66.43 \%$ with the particulate filter being limiting. The reason for this is the high stress on the particulate filter in urban drive cycles with low exhaust temperature resulting in difficulties for soot control. 


\begin{tabular}{|c|c|c|c|c|c|c|c|}
\hline customer segment & $\begin{array}{c}\text { market } \\
\text { share } \\
\text { annual } \\
\text { mileage } \\
\text { [kmiles] }\end{array}$ & 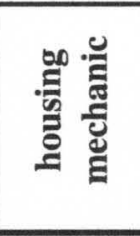 & 車 & 营 & 离 & 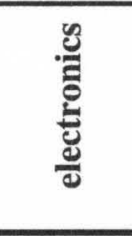 & 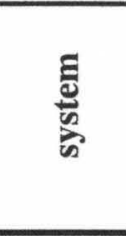 \\
\hline \multirow{2}{*}{ Long Haul } & $40 \%$ & \multirow{2}{*}{$83.92 \%$} & \multirow{2}{*}{$94.50 \%$} & \multirow{2}{*}{$79.63 \%$} & \multirow{2}{*}{$84.81 \%$} & \multirow{2}{*}{$83.77 \%$} & \multirow{2}{*}{$79.63 \%$} \\
\hline & 130 & & & & & & \\
\hline \multirow{2}{*}{ Pickup Heavy } & $15 \%$ & \multirow{2}{*}{$91.88 \%$} & \multirow{2}{*}{$95.29 \%$} & \multirow{2}{*}{$90.93 \%$} & \multirow{2}{*}{$91.61 \%$} & \multirow{2}{*}{$89.68 \%$} & \multirow{2}{*}{$89.68 \%$} \\
\hline & 80 & & & & & & \\
\hline \multirow{2}{*}{ Urban } & $10 \%$ & \multirow{2}{*}{$85.60 \%$} & \multirow{2}{*}{$95.29 \%$} & \multirow{2}{*}{$66.43 \%$} & \multirow{2}{*}{$77.76 \%$} & \multirow{2}{*}{$92.15 \%$} & \multirow{2}{*}{$66.43 \%$} \\
\hline & 60 & & & & & & \\
\hline \multirow{2}{*}{ Pickup Light } & $15 \%$ & \multirow{2}{*}{$91.88 \%$} & \multirow{2}{*}{$97.88 \%$} & \multirow{2}{*}{$82.11 \%$} & \multirow{2}{*}{$86.20 \%$} & \multirow{2}{*}{$94.70 \%$} & \multirow{2}{*}{$82.11 \%$} \\
\hline & 40 & & & & & & \\
\hline \multirow{2}{*}{ Construction } & $20 \%$ & \multirow{2}{*}{$78.71 \%$} & \multirow{2}{*}{$95.29 \%$} & \multirow{2}{*}{$90.93 \%$} & \multirow{2}{*}{$86.20 \%$} & \multirow{2}{*}{$94.70 \%$} & \multirow{2}{*}{$78.71 \%$} \\
\hline & 40 & & & & & & \\
\hline $\begin{array}{l}\text { LCL for comp./system } \\
\text { reliability across all } \\
\text { customer segments }\end{array}$ & & $85.43 \%$ & $95.36 \%$ & $82.64 \%$ & $85.61 \%$ & $89.32 \%$ & $80.00 \%$ \\
\hline
\end{tabular}

Table 7.4 Cumulative LCL of component and system reliability

Using equation (7.9) for each component the cumulative LCL of reliability, considering the share of the customer segments, can be calculated. The values of these cumulative LCLs of component reliability are given in the lowermost line of the matrix. For example the cumulative reliability particulate filter is $82.64 \%$.

$0.40 * 79.63 \%+0.15 * 90.93 \%+0.10 * 66.43 \%+0.15 * 82.11 \%+0.20 * 90.93 \%=82.64 \%$

The cumulative LCL of system reliability can be calculated with equation (7.10) by summing up the LCLs of system reliability in each customer segment weighted with the share of the segment. The cumulative LCL of system reliability for the warranty period $t_{0}=3$ years in this example yields to exactly $80.00 \%$.

$0.40 * 79.63 \%+0.15 * 89.68 \%+0.10 * 66.43 \%+0.15 * 82.11 \%+0.20 * 78.71 \%=80.00 \%$ 


\subsection{Integration of Customer Segment Consideration into RDT Optimization}

The models for the consideration of customer segments according to equation (7.9) and (7.10) can be combined with the optimization models for the RDT program. This of course adds complexity and can generate solution time difficulties.

There are many possible variations of such an extended optimization model. An example is given here:

- optimize the validation cost (minimize)

- meet a certain target for LCL of system reliability within each customer segment

- meet also a target for LCL of cumulative system reliability across all customer segments

- application of the improved optimization policy that allows for different test durations for the same test type as introduced in NLP (6.5)

The optimization model with respect to cost according to NLP of (6.2) including extensions like in NLP (6.5) are combined with the constraints of (7.9) and (7.10) as well as with the parameters from Table 7.2 and Table 7.3 for the consideration of customer segment specific stress factors.

The decision variables of this complex modified optimization problem are:

- duration of the planned tests according to test type $k: t_{P k}$

- sample size for the test according to test type $k: l_{P k}$

- duration of an additional planned test according to test type $k: t_{P^{*} k}$

- Boolean variable that indicates if one of the additional planned tests according to test type $k$ has a modified duration: $l_{P * k}$ 
The parameters of the NLP are:

- duration, for example the warranty period: $t_{0}$

- confidence level: $b$

- component Weibull shape parameter of component $i: \beta_{i}$

- information about the finished reliability tests:

test durations $t_{F i j}$ that indicate the relevant test time of test $j$ for component $i$ (remark: $t_{F i j}$ already considers acceleration factors $A F_{F i j}$ and the Boolean variable $o_{F i j}$ for indication if the test is obsolete or relevant for component $i$ )

- lower and upper bound for the test duration according to test type $k$ :

$t_{P, \min k}$ and $t_{P, \max k}$

- lower and upper bound for the sample size according to test type $k: l_{P, \min k}$ and $l_{P, \max k}$

- calculatory speed to transform the duration of test $k$ from a hourly base into a vehicle mileage equivalent: $v_{P k}$

- acceleration factor of test $k$ for component $i: A F_{P i k}$

- fixed cost of one test according to test type $k: C_{f k}$

- variable cost of test type $k$ for one unit of test duration (time or mileage): $C_{v k}$

- minimum cumulative LCL of system reliability across all customer segments $R_{S ; A S ; L C L ; b ; \min }$

- minimum LCL of system reliability in customer segment $l R_{S ; S M ~ l ; L C L ; b ; m i n}$

- annual mileage in customer segment $l d_{A} l$

- share of the customer segment $l$ in relation to the total production volume $S_{l}$

- $\quad$ stress factor $S F_{i l}$ of the customer usage in segment $l$ for component $i$

The following NLP (7.11) allows determining the RDT plan with optimal system reliability according to the improved optimization policy considering all $l_{\max }$ customer segments: 


\section{Minimize $C_{t}$}

subject to

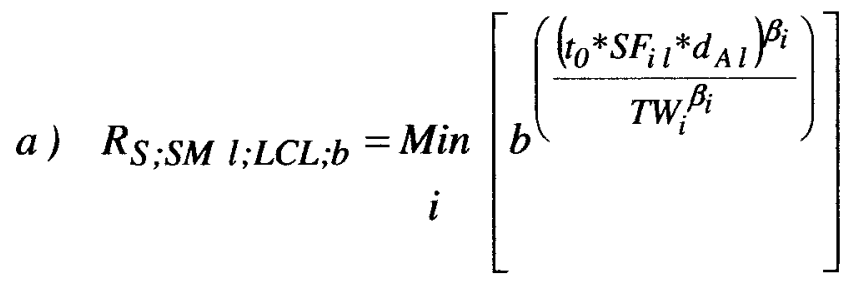

$$
\text { for } l=1, \ldots, l_{\max }
$$

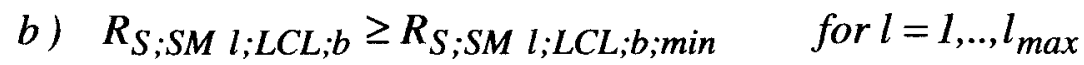

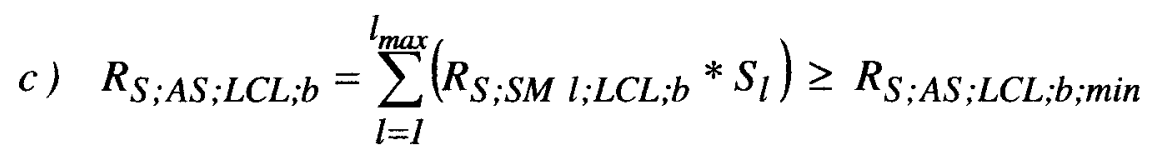

d) $T W_{i}^{\beta_{i}}=\sum_{j=1}^{m_{F}} t_{F i j} \beta_{i}+\sum_{k=1}^{m_{P}}\left[l_{P k} *\left(t_{P k} * v_{P k} * A F_{P i k}\right) \beta_{i}\right]$

$+\sum_{k=1}^{m_{P}}\left(l_{P^{*} k} * t_{P^{*} k} * v_{P k} * A F_{P i k}\right) \beta_{i} \quad$ for $i=1, \ldots, n$

e) $C_{t k}=\left(l_{P * k}+l_{P k}\right) *\left(C_{f k}+C_{v k} * t_{P k}\right) \quad$ for $k=1, . ., m_{P}$

f) $\sum_{k=1}^{m_{P}} C_{t k}=C_{t}$

g) $t_{P, \min k} \leq t_{P k} \leq t_{P, \max k} \quad$ for $k=1, . ., m_{P}$

h) $l_{P, \min k} \leq l_{P^{*} k}+l_{P k} \leq l_{P, \max k} \quad$ for $k=1, \ldots, m_{P}$

i) $l_{P k}=[0 ; 1 ; 2 ; 3 ; \ldots ..] ; l_{P^{*} k}=[0 ; 1] \quad$ for $k=1, \ldots, m_{P}$

j) $t_{0} ; A F_{P i k} ; v_{P k} ; \beta_{i} \geq 0 \quad$ for $i=1, \ldots, n ;$ for $k=1, \ldots, m_{P}$

k) $0 \leq b \leq 1$ 
Where:

a) calculation of the LCL for system reliability in all customer segments $l$, $R_{S ; S M l ; L C L ; b}$, according to (7.8).

b) the LCL for the system reliability in customer segment $l, R_{S ; S M l ; L C L ; b}$, have to be higher than a lower bound

c) calculation of the LCL for system reliability across all customer segments, $R_{S ; A S ; L C L ; b}$, according to (7.10). It has to be higher than a lower bound, $R_{S ; A S ; L C L ; b ; \min }$.

d) calculation of the Weibull equivalent single test duration $T W_{i}$ according to (3.5) for all components $i$ considering completed and future tests

e) calculation of the total cost (variable and fixed costs) for all tests $k$

f) calculation of the objective, the grand total cost of all tests

g) all tests $k$ meet the constraints on minimum and maximum duration

h) all tests $k$ meet the constraints on minimum and maximum sample size

i) the sample size of all tests $k$ are integer; there is either a single test of type $k$ with different duration or not.

j) the reference time $t_{0}$, each acceleration factor $A F_{i j}$, the calculatory speed $v_{P k}$ of each test $k$ and all Weibull parameters $\beta_{i}$ must not be negative

k) the confidence level $b$ must be between $0 \%$ and $100 \%$

The optimization models (6.1) and (6.3) trough (6.5) could be extended in a similar way as has happened here for model (6.2). As already mentioned for model (6.5) the additional aspects increase the complexity significantly and can make the solution of the mixed-integer NLP within finite computer time nearly impossible. 


\section{CONCLUSIONS}

\subsection{Overview}

The focus of this dissertation is on LCL of reliability of serial systems withWeibull components when the reliability demonstration testing (RDT) is conducted with zero failures. Methods for the calculation of LCL of reliability for Weibull have existed so far only for single items but not for systems. The usual methods for the parameter estimation of the underlying reliability functions like maximum likelihood estimator (MLE) or mean squares estimator (MSE) cannot be applied if the test data contains no failures.

Based on literature review as well as on additional research work, a first theorem for the LCL of system reliability of systems with Weibull components is formulated. It can be applied if testing is conducted with zero failures even if the Weibull shape parameters for components in the system are different. It also allows for different test durations the components were exposed to within the course of RDT. This could be a result from accelerated life testing (ALT) with different component specific acceleration factors. A second theorem for LCL of $B_{X}$ - lifetime, derived from the first theorem, has been formulated as well. Both theorems were mathematically proven for the general case of $n$ components.

The proven theorem is integrated into a model to analyze the sensitivity of the estimation of the Weibull shape parameter $\beta$. This model is also applicable if the Weibull parameter is subject to either total uncertainty or to uncertainty within a defined range. 
Furthermore the two proven theorems can be utilized as the core of RDT optimization models. These models ensure efficient RDT plans which means that the validation targets are achieved at the lowest possible cost. The optimization can be conducted with respect to different objectives such as reliability, $B_{X}$ - lifetime or validation cost. The necessary information on test cost or the possible range of test durations must be defined in a test procedure database.

The integration of the RDT optimization models mentioned before into the reliability growth process assures that the conducted RDT plan is still optimal and efficient even when boundary conditions have meanwhile changed such as test specific cost or failures that occurred. The necessary adaptations to the reliability growth process are presented in this work.

An enhanced methodology for the consideration of information about the customer segments including component specific stress levels for each segment is also presented. This methodology can be combined with the optimization models for RDT plans mentioned before.

The respective optimization models are mixed-integer and highly non-linear and therefore very difficult to solve. Within this research work the software package LINGO $^{\mathrm{TM}}$ was utilized to solve the models. The application of all optimization models is illustrated with examples. 


\subsection{Contributions to the Body of Knowledge}

This dissertation has added a framework to the body of knowledge that consists of methods for calculation of the LCL of reliability as well as of models for optimizing RDT plans. This framework can be applied to serial systems whose components follow a Weibull lifetime distribution when the RDT testing was conducted with zero failures. So far comparable models and methods did only exist for single items but not for systems. The main contributions are as follows:

- Introduction and proof of a new methodology to calculate the LCL of system reliability of a system with Weibull components that was tested with zero failures. This methodology is easy to apply since it uses only simple mathematical operations.

- Derivation of a method for the calculation of the LCL of $B_{X}$ - lifetime (of a system with Weibull components that was tested with zero failures) based on the method for LCL of system reliability.

- Presentation of an optimization model to account for the uncertainty of the estimation of the component Weibull parameters $\beta_{i}$ when the LCL of system reliability is calculated.

- Introduction of different optimization models to optimize RDT programs. The optimization can either take place with respect to system reliability, $B_{X}$ - lifetime or validation cost.

- A proposal of how to integrate the RDT optimization models within the reliability growth process to continuously review the RDT plan with respect to efficiency and achievement of the validation targets.

- Introduction of a model to consider customer segment specific load factors on each component of the system when LCL of system reliability in the entire population as well as in each customer segment is calculated.

- All models and methods are illustrated with examples. 


\subsection{Areas for Further Study}

Future research in this interesting area of LCL of reliability of system with Weibull components could investigate how the framework has to be enhanced if failures during RDT are allowed. Such a methodology would for example support decisions if countermeasures should be implemented at all, especially when a failure occurs late in the RDT and there are only few tests remaining to validate the modification.

Another interesting area of research in the context of this dissertation could be systems whose components have ith lifetime distribution functions other than Weibull like for example lognormal. To better fit the reliability model to the failure characteristics of the real technical system, a combination of different component lifetime distributions would be helpful.

The optimization models presented here are difficult to solve since they are based on very complex NLPs. The discontinuous minimum operation in combination with nonlinearity and integer constraints can lead to very long solution time with universal solver software packages. In many situations a close to optimal RDT plan would be sufficient if the solution time could be reduced significantly. Thus research in less time consuming optimization algorithms, for example genetic algorithms, could add a lot of value to this framework for LCL of reliability. 


\section{REFERENCES}

Allmen, C. R.; Lu, M.-W.: Sample Size for Failure Detection and Reliability

Demonstration, Proceedings of the Annual Reliability and Maintainability Symposium, Atlanta, GA , 1993

Ansell, J.; Walls, L.; Quickley, J.: Achieving Growth in Reliability, Annals of Operations Research, Vol. 91, p 11-24, 1999

Baklizi, A.; Ahmed, S.E.: On the Estimation of Reliability Function in a Weibull Lifetime Distribution, Statistics, Vol. 52, No. 4, p 351-362, 2008

Bailey, R. T.: Estimation from Zero-Failure Data, Risk Analysis, Vol. 17, No. 3, p 375-380, 1997

Bertsche, B.: Methods for Reliability Test Planning, chapter 8 in:

Reliability in Automotive and Mechanical Engineering, Springer Verlag, Berlin Heidelberg, 2008

Ebeling, C. E.: An Introduction to Reliability and Maintainability Engineering, Waveland Press, Long Grove IL, 2010

Elsayed, A. E.: Accelerated Life Testing, chapter 22 in Pham, H. (ed.): Handbook of Reliability Engineering, Springer Verlag, London, 2003

Gentle, J. E.: Random Number Generation and Monte Carlo Methods, $2^{\text {nd }}$ edition, Springer Verlag, New York, NY, 2003 
Hall, J. B.: Methodology for Evaluating Reliability Growth Programs of Discrete Systems, Dissertation, University of Maryland, 2008

Horngreen, C. T.; Sundem, G. L.; Stratton, W. O.; Burgstahler, D.; Schatzberg, J.: Introduction to Managing Accounting, $14^{\text {th }}$ edition, Pearson Education, Upper Saddle River, NJ, 2008

Huang, Z.; Porter, A. A.: Lower Bound on Reliability for Weibull Distribution when Shape Parameter is not Estimated Accurately, Proceedings of the Annual Reliability and Maintainability Symposium, Orlando, FL , 1991

Huang, Z.: Conditional Reliability Lower Bound for Weibull Distribution Without Estimating Shape Parameter, Proceedings of the Annual Reliability and Maintainability Symposium, Philadelphia, PA , 1997

Jiang, P.; Lim, J.H.; Zuo, M. J.; Guo, B.: Reliability Estimation in a Weibull Lifetime Distribution with Zero-failure Field Data,

Quality and Reliability Engineering International, Vol. 26, Issue 7, p 691-701, 2010

Ke, H.-Y.: Sampling Plans for Vehicle Component Reliability Demonstration, Quality and Reliability Engineering International, Vol. 15, p 363-368, 1999

Kim, M.; Yum, B.J.: Reliability Acceptance Sampling Plans for the Weibull Distribution Under Accelerated Type I Censoring, Journal of Applied Statistics, Vol. 36, Issue 1, p 11-20, 2009

Kleyner, A.; Boyle, J.: The Myths of Reliability Demonstration Testing, Test Engineering and Management, August/September, p 16-17, 2004 
Kleyner, A.; Sandborn, P.; Boyle, J.: Minimization of Life Cycle Costs Through Optimization of the Validation Program - A Test Sample Size and Warranty Cost Approach, Proceedings of the Annual Reliability and Maintainability Symposium, Los Angeles, CA , 2004

Kleyner, A.; Sandborn, P.: Minimizing Life Cycle Cost by Managing Product Reliability via Validation Plan and Warranty Return Cost, International Journal of Production Economics, Vol. 112, p 796-807, 2008

Klyatis, L. M..; Teskin, O. I.; Fulton, J. W.: Multi-Variate Weibull Model for Prediction System-Reliability from Testing Results of the Components, Proceedings of the Annual Reliability and Maintainability Symposium, Los Angeles, CA , 2000

LINDO $^{\circledR}:$ LING ${ }^{T M}$ user's guide, 13th edition, Lindo ${ }^{\circledR}$ Systems, Chicago, IL, 2011

Lu, M.-W.; Rudy, R. J.: Reliability Test Target Development, Proceedings of the Annual Reliability and Maintainability Symposium, Los Angeles, CA , 2000

Lu, M.-W.; Rudy, R. J.: Laboratory Reliability Demonstration Test Considerations, IEEE Transactions on Reliability, Vol. 50, No. 1, p 12-16, 2001

Lu, M.-W.; Wang, C. J.: Weibull Data Analysis with Few or No Failures, in: Recent Advances in Reliability and Quality in Design, editor: Pham, H., Springer, London, 2008

Military Handbook: Reliability Growth Management, US - Department of Defense, MIL-HDBK-189A (2009)

Montgomery, D. C.; Runger, G. C.: Applied Statistics and Probability for Engineers, $3^{\text {rd }}$ edition, John Wiley \& Sons, New York NY, 2003 
Nelson, W.: Weibull Analysis of Reliability Data With Few or No Failures, Journal of Quality Technology, Vol. 17, No. 3, p 140-146, 1985

Nicholls, D.; Lein, P.: Weibayes Testing: What is the Impact if Assumed Beta is Incorrect?, Proceedings of the Annual Reliability and Maintainability Symposium, Fort Worth, TX , 2009

O'Neill, T. S.: System Reliability Assessment from its Components, Journal of the Royal Statistical Society, Series C (Applied Statistics), Vol. 21, No. 3, 1972

Pham, H. (ed.): Springer Handbook of Engineering Statistics, Springer Verlag, London, 2006

Ramírez-Márquez, J. E.; Jiang, W.: On Improved Confidence Bounds for System Reliability, IEEE Transactions on Reliability, Vol. 55, No. 1, p 26-36, 2006

Tian, X.: Comprehensive Review of Estimating System-Reliability Confidence-Limits from Component-Test Data, Proceedings of the Annual Reliability and Maintainability Symposium, Seattle, WA , 2002

Wang, C. J.; Lu, M.-W.: A Two-Stage Sampling Plan for Bogey Tests, Quality and Reliability Engineering International, Vol. 8, p 29-35, 1992

Wang, C. J.: Sample Size Determination of Bogey Tests without Failures, Quality and Reliability Engineering International, Vol. 7, p 35-38, 1991

Yadav, O.P.; Singh, S.;Goel, P. S.: A Practical Approach to System Reliability Growth Modeling and Improvement, Proceedings of the Annual Reliability and Maintainability Symposium, Tampa, FL , 2003 
Yang, G.: Reliability Demonstration through Degradation Bogey Testing, IEEE

Transactions on Reliability, Vol. 58, p 604-610, 2009 


\section{APPENDIX 1 - THE WEIBULL DISTRIBUTION}

\section{The Weibull Distribution}

The Weibull distribution is widely used for reliability modeling. It has two parameters $\beta$ and $\theta$ and allows to fit to various shapes of real world failure distributions. For $\beta=1$ the Weibull distribution is identical with the exponential distribution; in this case the failure rate is constant. Values of $\beta<1$ lead to a decreasing failure rate and $\beta>1$ respectivly to an increasing failure rate.

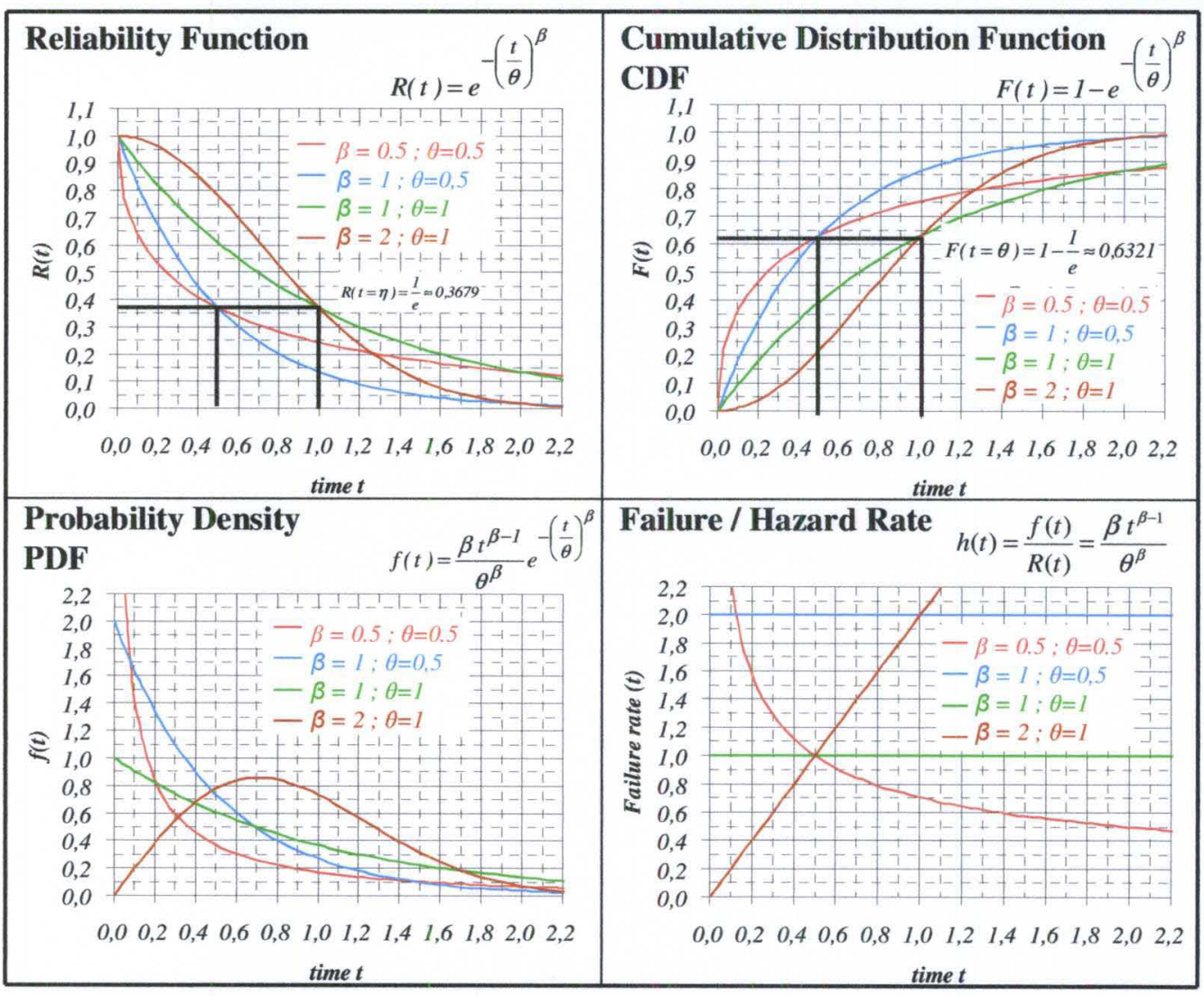

$$
\begin{aligned}
& M T T F=E(\tau)=\theta \Gamma\left(1+\frac{1}{\beta}\right) \text { with } \quad \Gamma(x)=\int_{0}^{\infty} y^{x-1} e^{-y} d y \\
& \operatorname{VAR}(\tau)=\sigma^{2}=\theta^{2}\left[\Gamma\left(1+\frac{2}{\beta}\right)-\Gamma\left(1+\frac{1}{\beta}\right)^{2}\right] \quad \text { parameter space: } t, \beta, \theta \geq 0
\end{aligned}
$$

Figure A1.1 Diagrams of the Weibull distribution (Reliability, CDF, pdf) 


\section{APPENDIX 2 - CHI-SQUARE FUNCT. (FOR $F=2$ )}

$\chi_{b ; f}^{2}$ is the right side $b^{\text {th }}$-percentile of the chi-square distribution with $f$ degrees of freedom. The chi-square-function is defined as follows ${ }^{21}$ :

$$
\begin{aligned}
& b=\int_{\chi_{b ; f}^{2}}^{\infty} g(x) d x=\int_{\chi_{b ; f}^{2}}^{\infty} \frac{1}{2^{f / 2} * \Gamma(f / 2)} * x^{(f / 2)-1} * e^{-x / 2} d x \\
& (0<b<1 ; f=1,2,3, \ldots)
\end{aligned}
$$

With the gamma function ([5] p. 131):

$$
\Gamma(r)=\int_{0}^{\infty} x^{r-1} e^{-x} d x \text { for } r>0
$$

For $f=2$ degrees of freedom:

$$
\Gamma(f / 2)=\Gamma(1)=\int_{0}^{\infty} x^{0} e^{-x} d x=\int_{0}^{\infty} e^{-x} d x=-\left[e^{-x}\right]_{0}^{\infty}=1
$$

Substituting (App.2.3) into (App. 2.1):

$$
\begin{aligned}
& b=\int_{\chi_{b ; f}^{2}}^{\infty} g(x) d x=\int_{\chi_{b ; 2}^{2}}^{\infty} g(x) d x=\int_{\chi_{b ; 2}^{2}}^{\infty} \frac{1}{2 * \Gamma(l)} * x^{(l)-1} * e^{-x / 2} d x \\
& b=\int_{\chi_{b ; 2}^{2}}^{\infty} \frac{1}{2} e^{-x / 2} d x=-\left[e^{-x / 2}\right]_{\chi_{b ; 2}^{2}}^{\infty}=e^{-\chi_{b ; 2}^{2} / 2} \\
& \chi_{b ; 2}^{2}=-2 \ln b=2 \ln (1 / b)
\end{aligned}
$$

${ }^{21}$ see: Montgomery and Runger (2003) p. 262 


\section{APPENDIX 3 - RELIABILITY OF A SYSTEM WITH $\boldsymbol{N}$ IDENTICAL COMPONENTS}

A system with $n$ identical component each with Weibull parameter $\beta$ was tested in $m$ tests each with test time $t_{T}$.

What is the $b=0.10 \mathrm{LCL}$ of reliability of the system $R_{S ; L C L ; b}$ at time $t_{0}$ ?

\section{$\underline{\text { I System View }}$}

According to (3.5) the Weibull equivalent single test duration $T W_{S}$ for the system is:

$T W_{s}=\sqrt[\beta]{t_{T}^{\beta} * m}=t_{T} * \sqrt[\beta]{m}$

The $b=0.10 \mathrm{LCL}$ of reliability of the system $R_{S ; L C L ; b}$ at time $t_{0}$ is (see (3.7)):

$R_{S ; L C L ; b}\left(t_{0}\right)=e^{\left(\frac{t_{0}^{\beta} * \ln (b)}{T W_{S}^{\beta}}\right)}$

(App.3.2)

For $b=0.10 \ln (b)$ yields to -2.3026 . Plugged into (App.3.2):

$R_{S ; L C L ; b}\left(t_{0}\right)=e^{\left(\frac{t_{0}^{\beta} * \ln (b)}{T W_{S}^{\beta}}\right)}=e^{\left(\frac{t_{0}^{\beta} *(-2.30)}{T W_{S}^{\beta}}\right)}$

(App.3.3) 


\section{Component View}

In each test $n$ components were tested. Therefore, the Weibull equivalent single test duration $T W_{\text {Comp }}$ for the components is:

$T W_{\text {comp }}=\sqrt[\beta]{t_{T}^{\beta} * m * n}=T W_{S} * \sqrt[\beta]{n}$

The $b=0.10 \mathrm{LCL}$ of reliability of each of the components $R_{L C L ; b ; c o m p}$ at time $t_{0}$ is:

$R_{L C L ; b ; c o m p}\left(t_{0}\right)=e^{\left(\frac{t_{0}^{\beta} * \ln (b)}{T W_{c o m p}^{\beta}}\right)}=e^{\left(\frac{t_{0}^{\beta} * \ln (b)}{T W_{S}^{\beta} * n}\right)}=e^{\left(\frac{t_{0}^{\beta} *[\ln (b)] / n}{T W_{S}^{\beta}}\right)}$

(App.3.5)

The system consists of $n$ identical components in a serial configuration.

The system reliability is:

$R_{S ; L C L ; b}\left(t_{0}\right)=R_{L C L ; b ; c o m p}^{n}\left(t_{0}\right)=\left[e^{-\left(\frac{t_{0}^{\beta} *[\ln (l / b)] / n}{T W_{s y s}^{\beta}}\right)}\right]^{n}=e^{-\left(\frac{t_{0}^{\beta} \ln (l / b)}{T W_{s y s}^{\beta}}\right)}($ App.3.6)

Both views yield the same LCL of the system reliability as shown in (App.3.3) and (App.3.6). 


\section{Determination of System LCL According to the Proposed Theorem}

The $n$ components were exposed to $T W_{i}$ during RDT and no failure occurred. $T W_{S}$ is equal to $T W_{i}$ for all components.

Each individual component has a LCL of reliability according to:

$R_{L C L ; i}\left(t_{0}\right)=e^{\left(\frac{t_{0}^{\beta} * \ln (b)}{T W_{i}^{\beta}}\right)}$

The lowest of these reliability values is the equal to the LCL of system reliability $R_{S ; L C L ; b}\left(t_{0}\right)$ according to equation (3.15).

In this example all component LCL $R_{L C L, b, i}$ are identical (assumption of identical components). The LCL of system reliability yields:

$$
R_{S, L C L, b}\left(t_{0}\right)=\operatorname{Min}_{i}\left[R_{L C L ; i}\left(t_{0}\right)\right]=\operatorname{Min}_{i}\left[e^{\left(\frac{t_{0}^{\beta} * \ln (b)}{T W_{i}^{\beta}}\right)}\right]=e^{\left(\frac{t_{0}^{\beta} * \ln (b)}{T W_{S}^{\beta}}\right)}
$$

Approach III yield the same LCL of system reliability as calculated in (App.3.3). 


\section{APPENDIX 4 - LCL OF RELIABILITY OF SINGLE WEIBULL ITEMS WITH ZERO FAILURES}

The lower confidence limit for the reliability of single items that are tested with zero failures according to equation (3.9) is:

$$
R_{L C L, b}\left(t_{0}\right)=e^{\left(\frac{t_{0}^{\beta} * \ln (b)}{T W^{\beta}}\right)}=b^{\left(\frac{t_{0}^{\beta}}{T W^{\beta}}\right)}
$$

This can be derived as follows:

Let $R_{a}$ denote the actual reliability of the item and $\theta_{a}$ the respective scale parameter. The item follows the Weibull law the according to equation (2.11). So the respective reliability at time $t_{0}$ yields:

$$
R_{a}\left(t_{0}\right)=e^{-\left(\frac{t_{0}}{\theta_{a}}\right)^{\beta}}
$$

The probability of zero failure occurring during the entire RDT program with $m$ tests is:

$$
\operatorname{Pr}(\text { O failures })=\prod_{j=1}^{m} e^{-\left(\frac{t_{j}}{\theta_{a}}\right)^{\beta}}=e^{-\sum_{j=h}^{m}\left(\frac{t_{j}}{\theta_{a}}\right)^{\beta}}=e^{-\left(\frac{T W}{\theta_{a}}\right)^{\beta}}=e^{-\frac{T W^{\beta}}{\theta_{a}^{\beta}}}
$$

To prove (App.4.1) it has to be shown that the probability of zero failure occurring, or a type II error respectively, is less than $b$ if the actual reliability is less or equal than $R_{L C L, b}$ : 
$\operatorname{Pr}($ O failures $)=e^{-\frac{T W^{\beta}}{\theta_{a}^{\beta}}} \leq b$

(App.4.4)

$R_{a}\left(t_{0}\right)=e^{-\left(\frac{t_{0}}{\theta_{a}}\right)^{\beta} !}=R_{L C L, b}\left(t_{0}\right)=e^{\left(\frac{t_{0}^{\beta * \ln (b)}}{T W^{\beta}}\right)}$

(App.4.5)

with $0 \leq b \leq 1 ; t_{0}, \theta_{a}, T W, \beta>0$

Equation (App.4.5) can be transformed in the following way:

$$
\begin{aligned}
& e^{-\left(\frac{t_{0}}{\theta_{a}}\right)^{\beta}}=e^{-\left(\frac{t_{0}^{\beta * \ln (l / b)}}{T W^{\beta}}\right)} \Leftrightarrow\left(\frac{t_{0}}{\theta_{a}}\right)^{\beta}=\left(\frac{t_{0}^{\beta} * \ln (1 / b)}{T W^{\beta}}\right) \\
& \frac{t_{0}}{\theta_{a}}=\frac{t_{0}}{T W} * \ln (1 / b)^{I / \beta} \Leftrightarrow \theta_{a}=\frac{T W}{\ln (1 / b)^{1 / \beta}}
\end{aligned}
$$

Substituting (App.4.6) into equation (App.4.4):

$$
\begin{gathered}
e^{-\frac{T W^{\beta}}{\theta_{a}^{\beta}}}=e^{-\frac{T W^{\beta}}{\left[\frac{T W}{\ln (1 / b)^{1 / \beta}}\right]^{\beta}} \leq b} ! \\
e^{-\frac{T W^{\beta} * \ln (1 / b)}{T W^{\beta}}}=e^{\ln (b)}=b \leq b
\end{gathered}
$$

It is obvious that the inequality (App.4.7) is true for all $0 \leq b \leq 1$. This means that equation (App.4.1) is mathematically proven. 
If the Weibull formula of (2.10) is applied to (App.4.1) the $b$ LCL of the Weibull scale parameter $\theta_{L C L ; b}$ can be calculated:

$R_{L C L, b}\left(t_{0}\right)=e^{\left(\frac{t_{0}^{\beta} * \ln (b)}{T W^{\beta}}\right)}=e^{-\left(\frac{t_{0}^{*} \ln (1 / b)^{l / \beta}}{T W}\right)^{\beta}}=e^{-\left(\frac{t_{0}}{\theta_{L C L ; b}}\right)^{\beta}}$

Taking the $\log$ on both sides and solving the equation for $\theta_{L C L ; b}$ results in:

$$
\theta_{L C L ; b}=\frac{T W}{\ln (1 / b)^{1 / \beta}}
$$

The $B_{X}$ - lifetime of a Weibull item is defined as $^{22}$ :

$$
B_{X}=\theta *\left[1-\frac{X}{100 \%}\right]^{1 / \beta}
$$

Substituting equation (App.4.8) into (App.4.9) leads to:

$$
B_{X ; L C L ; b}=\theta_{X ; L C L ; b} *\left[1-\frac{X}{100 \%}\right]^{1 / \beta}=\frac{T W *\left[1-\frac{X}{100 \%}\right]^{1 / \beta}}{\ln (1 / b)^{1 / \beta}}
$$

\footnotetext{
${ }^{22}$ see equation (2.15) in chapter 2.1
} 


\title{
APPENDIX 5 - ON LCL OF SYSTEM RELIABILITY WHEN FAILURES HAVE OCCURRED IN RDT
}

\author{
Summary \\ In addition to the framework explained in detail for zero failures occurring in RDT, it was \\ investigated whether a similar approach is also possible when there are failures in RDT. \\ A similar approach in this context means that it was especially analyzed if the LCL of \\ system reliability is also equal to the lowest LCL of component reliability when there \\ were failures occurring during RDT.
}

The outcome of this investigation is:

When there are failures occurring in RDT, the lowest LCL of component reliability is not an adequate expression for the LCL of system reliability.

\section{LCL for Component Reliability with Failures in RDT}

The test data in this context including some failures can be classified as "multiply censored data" since the number of failures is a random variable (which would not be the case for type II censoring) and the length of the testing is not necessarily pre-defined at the beginning of the testing (as it would be for the case of type I censoring). If there is a failure of one component, then the testing of the entire system will be stopped for the entire system independently from the initially planned test duration. For more details on censored data, see section 2.5.1. 
The starting point is the set of equations (2.32) and (2.33) in Chapter 2.5.2 for the $b \mathrm{LCL}$ of the Weibull scale parameter $\theta_{L C L ; b}$ and the $b$ LCL of reliability $R_{L C L ; b}$. Although these equations were originally dedicated to single items, they will be applied here to components of a system. By substituting the number of failures $r_{i}$ that occurred during RDT on component $i$ and the Weibull equivalent single test duration $T W_{i}$ into these equations, the $b$ LCL of the component Weibull scale parameter $\theta_{C i ; L C L ; b}$ and the $b \mathrm{LCL}$ of component reliability $R_{C i ; L C L ; b}$ is found to be:

$$
\begin{aligned}
& \theta_{C i ; L C L ; b}=\left(\frac{2 \sum_{j=1}^{m} t_{i j}^{\beta_{i}}}{\chi_{b ; 2 r_{i}+2}^{2}}\right)^{l / \beta_{i}}=\left(\frac{2 * T W_{i}^{\beta_{i}}}{\chi_{b ; 2 r_{i}+2}^{2}}\right)^{I / \beta_{i}} \\
& R_{C i ; L C L ; b}\left(t_{0}\right)=e^{-\left(\frac{t_{0}}{\theta_{C i ; L C L ; b}}\right)^{\beta_{i}}}=e^{-\left(\frac{t_{0}^{\beta_{i} * \chi_{b ; 2 r_{i}+2}^{2}}}{2 * T W_{i}^{\beta_{i}}}\right)}
\end{aligned}
$$

According to the definition of LCL, the probability of observing $r_{i}$ or less failures during the entire RDT program will be lower than $b$ if the actual value of component reliability is equal to $R_{C i, L C L ; b}$ (see Chapter 4.1 ).

Let $\theta_{a ; C i}$ be the actual value of the component Weibull scale parameter. The probability that $r_{i}$ or less failures occur during RDT is $p_{r}$. Substituting both into (App.5.1) leads to:

$$
\theta_{a ; C i}=\left(\frac{2 * T W_{i}^{\beta_{i}}}{\chi_{p_{r i} ; 2 r_{i}+2}^{2}}\right)^{1 / \beta_{i}}
$$

Solving equation (App.5.3) for $p_{r i}$ requires the utilization of tables for the $\chi^{2}$-distribution or the respective $\chi^{2}$-functions in mathematical software packages like LINGO $^{\mathrm{TM}}$ or EXCEL since there is no mathematical way to calculate $p_{r i}$ directly. 


\section{A Potential LCL of System Reliability based on the LCLs of Component Reliability}

As discussed in Chapter 3.3, we assume a serial system consisting of $n$ Weibull components, each with shape parameter $\beta_{i}$. Its components are exposed to different tests that can be summarized per component in the Weibull equivalent single test duration $T W_{i}$ (see definitions in equations (3.4) or (3.5)). During RDT $r_{i}$ failures are occurring on each component $i$. It is now investigated according to the first theorem (see equation (3.15) in Chapter 3.3), whether the $b$ LCL of system reliability $R_{S ; L C L ; b}$ is also equal to the lowest one of the LCLs for component reliability (remark: the outcome of the investigation was negative).

$$
R_{S ; L C L ; b}\left(t_{0}\right)=\operatorname{Min}_{i} R_{C i, L C L ; b}\left(t_{0}\right)=\operatorname{Min}_{i}\left[e^{-\left(\frac{t_{0}^{\beta_{i} * \chi_{b ; 2 r_{i}+2}^{2}}}{2 * T W_{i}^{\beta_{i}}}\right)}\right]
$$

Per definition of the LCL, the probability of observing $r_{i}$ or less failures on all $n$ components should be less or equal than $b$ if the actual reliability of the serial system with Weibull components is less or equal to the LCL of system reliability.

This means that the actual system reliability is higher than the LCL of system reliability if the probability of observing $r_{i}$ or less failures is equal to $b$ :

$$
\begin{aligned}
& \prod_{i=1}^{n} p_{r i}=b \quad \text { with } p_{r i} \geq b \quad \text { for all } i \\
& R_{a ; S}=e^{-\sum_{i=1}^{n}\left(\frac{t_{0}}{\theta_{a ; i}}\right)^{\beta_{i}}}=e^{-\sum_{i=1}^{n}\left(\frac{t_{0}^{\beta_{i} * \chi_{p_{r i} ; 2 r_{i}+2}^{2}}}{2 * T W_{i}^{\beta_{i}}}\right)} \geq R_{S ; L C L ; b}\left(t_{O}\right)
\end{aligned}
$$




$$
e^{-\sum_{i=1}^{n}\left(\frac{t_{0}^{\beta_{i} * \chi_{p}^{2} ; 2 r_{i}+2}}{2 * T W_{i}^{\beta_{i}}}\right)} \geq \operatorname{Min}_{i}\left[e^{-\left(\frac{t_{0}^{\beta_{i} * \chi_{b ; 2 r_{i}+2}^{2}}}{2 * T W_{i}^{\beta_{i}}}\right)}\right]
$$

The "lifetime ratio" $L R$, that brings test duration into a relation with the reference duration $t_{0}$, was already introduced in Chapter 2.5.2. Here $L R$ is applied to the Weibull equivalent single test duration $T W_{i}$. Utilizing this approach, it is possible to even further simplify the expression above with the introduction of $Y_{i}$ as follows:

Let $Y_{i}$ be: $Y_{i}=\frac{2 * T W_{i}^{\beta_{i}}}{{ }_{t}^{\beta_{i}}}=2 * L R^{\beta_{i}}$ with $Y_{i}>0$. Substituting this into (App.5.6):

$$
e^{-\sum_{i=1}^{n}\left(\frac{\chi_{p_{r i} ; 2 r_{i}+2}^{2}}{Y_{i}}\right)} \geq \operatorname{Min}_{i}\left[e^{-\left(\frac{\chi_{b ; 2 r_{i}+2}^{2}}{Y_{i}}\right)}\right]
$$

Taking the $\log$ on both sides and multiplying by $-l$ leads to the following expression:

$$
\begin{aligned}
& \sum_{i=1}^{n}\left(\frac{\chi_{p_{r i} ; 2 r_{i}+2}^{2}}{Y_{i}}\right) \leq \operatorname{Max}_{i}\left(\frac{\chi_{b ; 2 r_{i}+2}^{2}}{Y_{i}}\right) \\
& \prod_{i=1}^{n} p_{r i}=b
\end{aligned}
$$

It is not possible to solve this expression mathematically since there will be no direct solution if there are failures and thus the degree of freedom of the $\chi^{2}$-distribution is higher than 2. So this will be further investigated with the help of Monte Carlo analyses if the expression is true in all cases. 
The potential theorem (App.5.4) can be proven if the expression (App.5.8) is a correct statement for all cases when the following variables are within their defined ranges:

- $Y_{i}>0$

- $0<p_{r i}<1$

- $r_{i}=0,1,2, \ldots$

\section{MCS Analysis to Prove the Potential LCL of System Reliability}

To investigate whether the expression in (App.5.8) is a valid statement for the entire definition range of the variables, a Monte Carlos Simulation (MCS) with recursive random number generation was conducted. The calculation was done with LINGO $^{\mathrm{TM}}$. The parameters of the MCS were as follows:

- 100 repetitions

- $2 \leq n \leq 10$ components

- $0 \leq r_{i} \leq 10$ failures on component $i$

- $l \leq Y_{i} \leq 100$ as a representation of the testing duration related to $t_{0}$

- $0.005 \leq p_{r i} \leq 0.99$

- recursive random number generation with LINGO $^{\mathrm{TM}}$

In 47 of the 100 repetitions of the MCS the in-equation of (App.5.8) was not fulfilled, i.e. the sum on the left side of the in-equation was higher than the potential LCL. This means that only in roughly the half of the investigated cases the actual system reliability was higher than the potential $b$ LCL of the system reliability given the case that the probability of $r_{i}$ or less failures on all components is exactly $b$. This is also illustrated in Figure A5.1: All repetitions that lead to a negative "term" are violating the mentioned in-equation and are marked as bold. The repetitions in white are meeting the in-equation. 


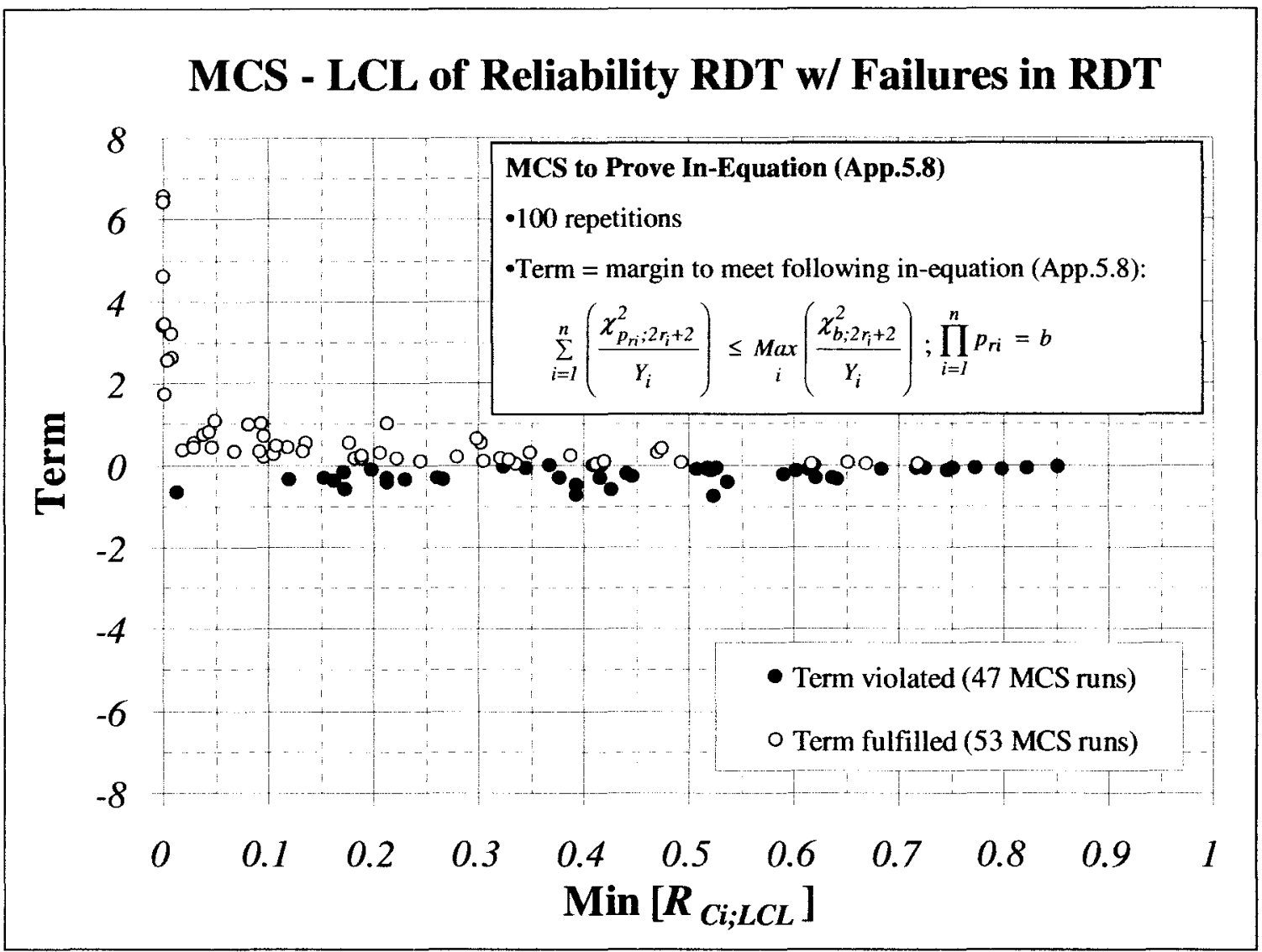

Figure A5.1 MCS result: the potential LCL of system reliability is not adequate

The consequence of this result of the MCS is:

When there are failures occurring in RDT, the lowest LCL of component reliability is not the LCL of system reliability.

For the zero failure case $\left(\sum r_{i}=0\right)$ a MCS analyses with even 50,000 repetitions was conducted. Not a single case was observed, where the in-equation (App.5.8) has been violated. This is not surprising since the statement for the LCL of system reliability was even proved mathematically in Chapter 4. 


\section{Consequences for the LCL of System Reliability with Failures During RDT}

It is obvious that the expression (App.5.8) is not adequate. The components, that are not the limiting on in this expression but still have failures, are not considered to the required content when the LCL of system reliability is determined.

In order to get a more conservative expression for the LCL of system reliability in the case of failures during RDT, a correction factor $C F$ (with $C F \leq 1$ ) has to be introduced. This correction factor reduces the LCL of system reliability dependent on the following arguments:

- $r_{i}$ - the number of failures observed on each component $(i=1, \ldots, n)$

- $Y_{i}-$ representing the extent of testing in relation to the duration $t_{0}$ for each component $(i=1, \ldots, n)$

- $\quad b$ - the confidence level

The determination of the correction factor is not considered to be part of this dissertation since it is focused on RDT with zero failures. This is subject to future research. 


\section{APPENDIX 6 - APPLIED COMPUTER SYSTEM TO SOLVE OPTIMIZATIONS}

\section{Utilized Optimization Software Package}

LINGO $^{\text {TM }} 13$ (13.0.2.10) from LINDO ${ }^{\circledR}$ Systems Inc., Chicago IL

\section{PC Operating System}

Windows 7 Home Premium (German Version)

\section{PC Hardware}

Medion Akoya E7214

32 bit

RAM 3.00 GByte

Processor: Intel ${ }^{\circledR}$ Core $^{\mathrm{TM}}$ i3 CPU M350 2.27/2.26 GHz

(Notebook with 2010 Hardware) 


\section{APPENDIX 7 - LIST OF ABBREVIATIONS}

$\begin{array}{ll}\text { ALT } & \text { Accelerated Lifetime Test } \\ \text { CDF } & \text { Cumulative Distribution Function } \\ \text { LCL } & \text { Lower Confidence Limit } \\ \text { LP } & \text { Linear Program } \\ \text { MCS } & \text { Monte Carlo Simulation } \\ \text { NLP } & \text { Non-Linear Program } \\ \text { pdf } & \text { Probability Density Function } \\ \text { RDT } & \text { Reliability Demonstration Testing }\end{array}$




\title{
CURRICULUM VITAE
}

\author{
Name Markus Willi Kemmner \\ Address $\quad$ Braikestrasse 9 \\ 72669 Unterensingen (Germany) \\ DOB Unterensingen / Germany - March 21, 1971 \\ Marital Status married since 1998, 5 children $(1999,2002,2005,2007,2009)$ \\ Education Berufsakademie Stuttgart (1991 - 1994) \\ Dipl-Ing.(BA) Mechanical Engineering \\ Technische Universität Dresden (1995-1998) \\ Dipl-Ing. Mechanical Engineering / Automotive Engineering \\ Fernuniversität Hagen (1997 - 2002) \\ Dipl.-Wirt.Ing. Economics, Business Administration \\ University of Louisville (UofL), Louisville KY (since 2009) \\ German PhD program Industrial Engineering \\ Professional Daimler-Benz, DaimlerChrysler, Daimler (since 1994): \\ Career \\ Component Test Engineer for Truck Engines (5 years) \\ Project Manager R\&D for "Increased Displacement for MD Engines" \\ (1 year) \\ Manager R\&D Performance, Combustion and Emissions MD Truck \\ Engines (2.5 years) \\ Senior Manager R\&D Performance, Combustion and Emissions MD \\ Truck Engines ( 3 years) \\ Senior Manager R\&D Exhaust Aftertreatment Engineering for Truck \\ Engines (6 years) \\ Senior R\&D Project Leader for a New Generation of Passenger Car \\ Diesel Engines and Powertrain (since 2012)
}

